\title{
Revision of the Baeoglena species of the West Palaearctic Region (Coleoptera: Staphylinidae: Aleocharinae)
}

With 67 figures and 7 maps

VOLKER ASSING ${ }^{1}$

${ }^{1}$ Gabelsbergerstraße 2, 30163 Hannover, Germany. - vassing.hann@t-online.de Published on 2019-06-24

DOI:10.21248/contrib.entomol.69.1.001-032

\begin{abstract}
The subgenus Baeoglena Thomson, 1867 of the speciose aleocharine genus Oxypoda Mannerheim, 1830 has been subject to considerable taxonomic confusion rendering a reliable identification of material from regions other than Central Europe and the Canary Islands virtually impossible. Based on a revision of abundant material from various major public and private collections, seven species are distributed in the West Palaearctic region exclusive of the Canary Islands. Two new species are described and illustrated: Oxypoda (Baeoglena) rectacia spec. nov. (East Mediterranean, from South Greece to the Middle East) and O. (B.) derecta spec. nov. (West Caucasus, Northeast Anatolia). Diagnoses and illustrations of the genitalia are provided for the remaining five species. The following synonymies are established: Oxypoda nova Bernhauer, $1902=$ O. giachinoi PACE, 2001, syn. nov.; O. hispanica FAGEL, 1958 and its replacement name O. inexpectata FAGEL, 1965 are removed from synonymy with O. praecox ERICHSON, 1839 and synonymized with O. fusina Mulsant \& Rey, 1875. Oxypoda recondita KraAtz, 1856 and O. dalmatina Bernhauer, 1905 are excluded from Baeoglena and moved to the subgenus Bessopora Thomson, 1859. Lectotypes are designated for Oxypoda nova Bernhauer, 1902, O. caucasica Bernhauer, 1902, and O. kuehnelti Scheerpeltz, 1963. An outline of the taxonomic history, a diagnosis of the subgenus, a checklist, and a key to species are provided. A zoogeographic analysis revealed some remarkably discontinuous distributions and parapatric distribution patterns which are plausibly explained only with interspecific competition among Baeoglena species. The distributions of the subgenus as a whole and of the individual species in the West Palaearctic region are mapped.
\end{abstract}

\section{Taxonomic acts}

Oxypoda rectacia spec. nov. - urn:lsid:zoobank.org:act:8E5A6F28-9AD3-474E-99F8-9CBA0F85C4DC

Oxypoda derecta spec. nov. - urn:lsid:zoobank.org:act:4C73E05F-20F7-4697-A4BA-D9E030B28A27

\section{Key words}

Oxypoda, Baeoglena, West Palaearctic region, taxonomy, new species, new synonymies, lectotype designations, zoogeography, interspecific competition, distribution maps, checklist, key to species 


\section{Zusammenfassung}

Die Untergattung Baeoglena Thomson, 1867 der artenreichen Aleocharinengattung Oxypoda Mannerheim, 1830 war bisher taxonomisch weitgehend ungeklärt. Eine verlässliche Determination von Tieren, die nicht von den Kanarischen Inseln oder aus Nord- und Mitteleuropa stammten, war daher praktisch unmöglich. Eine Revision umfangreichen Materials aus einer Reihe von öffentlichen und privaten Sammlungen ergab, dass die Untergattung in der Westpaläarktis, ausgenommen die Kanarischen Inseln, mit sieben Arten vertreten ist. Zwei Arten werden erstmals beschrieben und abgebildet: Oxypoda (Baeoglena) rectacia spec. nov. (östlicher Mittelmeerraum von Südgriechenland bis zum Nahen Osten) und $O$. (B.) derecta spec. nov. (Westkaukasus, Nordostanatolien). Kurzdiagnosen der übrigen fünf Arten werden durch Genitalabbildungen illustriert. Folgende Synonymisierungen werden vorgenommen: Oxypoda nova Bernhauer, 1902 = O. giachinoi PACE, 2001, syn. nov.; das primäre Homonym O. hispanica FAgeL, 1958 und sein Ersatzname O. inexpectata FAgEL, 1965, bisher Synonyme von O. praecox ERICHsON, 1839, werden mit O. fusina Mulsant \& Rey, 1875 synomymisiert. Oxypoda recondita KraAtz, 1856 und O. dalmatina Bernhauer, 1905 gehören nicht zu Baeoglena und werden der Untergattung Bessopora Thomson, 1859 zugeordnet. Für Oxypoda nova Bernhauer, 1902, O. caucasica Bernhauer, 1902 und O. kuehnelti Scheerpeltz, 1963 werden Lektotypen designiert. Die Revision wird durch eine Zusammenfassung der taxonomischen Geschichte und einer Diagnose der Untergattung sowie durch einen Katalog und eine Bestimmungstabelle ergänzt. Eine zoogeographische Analyse ergab einige bemerkenswert disjunkte Verbreitungsbilder und parapatrische Verbreitungsmuster, die plausibel nur mit interspezifischer Konkurrenz unter den Baeoglena-Arten erklärt werden können. Die Verbreitungsgebiete der in der Westpaläarktis (ohne die Kanarischen Inseln) vertretenen Arten werden anhand von Karten illustriert.

\section{Schlüsselwörter}

Oxypoda, Baeoglena, Westpaläarktis, Taxonomie, neue Arten, neue Synonymien, Lektotypendesignationen, Zoogeographie, interspezifische Konkurrenz, Verbreitungskarten, Katalog, Bestimmungstabelle

\section{Introduction}

Thомson (1867) originally described Baeoglena as a genus to accommodate only Oxypoda praecox ERICHSON, 1839, the type species by monotypy. Mulsant \& Rey (1875) treated Baeoglena as a subgenus of Oxypoda Mannerheim, 1830, at that time including seven species: O.praecox, O. exoleta ERICHSON, 1839, O. longula Brisout de BARneville, 1863 (today a synonym of O. lurida Wollaston, 1857), O. flava KraAtZ, 1856 (today a synonym of O. soror Thomson, 1855), O. bicolor Mulsant \& Rey, 1853, O.fusina Mulsant \& Rey, 1875 and O. breviuscula Mulsant \& Rey, 1875. FAuvel (1895) synonymized O. breviuscula with $O$. fusina and regarded the latter as a variety of O. praecox. In his synopsis of the Oxypoda species known from the Palaearctic region at that time, Bernhauer (1902) retained only O. praecox, O. lurida, and O. fusina (with O. breviuscula and O. corsica BERNHAUER, 1899 as junior synonyms) in the subgenus and added O. recondita KraAtz, 1856 and two newly described species: O. caucasica and O. nova. Bernhauer (1905) described O. dalmatina and assigned it to Baeoglena based on its similarity to O. recondita.

FAgEL (1958) described O. (Baeoglena) hispanica from Spain, a preoccupied name FAGEL (1965) subsequently replaced with the nomen novum $O$. inexpectata. This name, however, was synonymized with O. praecox by Tronquet (1999).

In his revision of Canarian Oxypoda MANNERHEIM, 1830, ZERCHE (1996) recognized six Baeoglena species, all of them island or even local endemics confined to Tenerife and La Gomera, O. brevipennis Wollaston, 1864 and five newly described (O. gillerforsi, O. hernandezi, O. meybohmi, O. tenoensis, and O.wunderlei). He also moved O.lurida from Baeoglena to Atlantoxypoda ZERCHE, 1996.

Thus, Smetana (2004) lists a total of 13 valid Baeoglena species (with four synonyms), including O. giachinoi, which PACE (2001) had erroneously attributed to the subgenus Mycetodrepa Thomson, 1859. No significant changes were made in the most recent edition of the Palaearctic Catalogue (Schülke \& SMetana 2015) with one exception: Assing (2005) had described a seventh Canarian species from La Gomera, O. caeca, the sole subanophthalmous representative of the subgenus.

Unlike the Baeoglena species of the Canary Islands, those from the remainder of the West Palaearctic region have never been revised, neither taxonomically nor zoogeographically.

The distributions compiled in Schülke \& Smetana (2015) are remarkably implausible. Three species (O. kuehnelti, O. giachinoi, O. dalmatina) have been recorded only from Greece or Croatia (in their respective original descriptions), although they do not possess any of the adaptations (reduced eye size; reduced wings) usually encountered in locally or regionally endemic species. Other more frequently recorded species, on the other hand, are given as widespread with largely overlapping ranges. Oxypoda praecox is listed for most of South, 
Central, and North Europe, from Portugal in the west to Ukraine in the east and recorded even from West and East Siberia. The distributions of O. fusina and O. nova largely overlap with that of O. praecox in South Europe and range from Spain to Turkey. Finally, O. caucasica is listed for the Caucasus region (Azerbaijan, Georgia, Russian South European territory, Iran) and Germany (sic!).

The main reason for this situation is that the sexual characters of the continental species have never been studied systematically and that nearly all previous records are based on external characters such as size, coloration, the length of the elytra, and the density of the punctation of the abdomen (see BERNHAUER 1902). Illustrations of the genitalia available in the literature are confined to drawings or rough sketches of O. praecox, O. fusina, O. inexpectata (synonym), and O.giachinoi (LoHSE 1974, PACE 2001, Strand \& Vik 1966, Tronquet 1999, ZERCHE 1994). As a consequence, a reliable identification of Baeoglena species, except $O$. praecox from Central Europe, was practically impossible, so that records of this subgenus were often given as Oxypoda (Baeoglena) sp. in recent articles (e.g., Assing 2013, 2017).

It is the objective of the present article to clarify the status of the available names, the identities of the species, and their distributions, based on a thorough revision of types and additional material.

\section{Material and methods}

The material treated in this study is deposited in the following public and private collections:

FMNH Field Museum of Natural History, Chicago (C. Maier, A. Newton)

HNHM Hungarian Natural History Museum, Budapest (Gy. Makranczy)

MHNG Muséum d'Histoire Naturelle, Genève (G. Cuccodoro)

MNB Museum für Naturkunde, Berlin (incl. coll. Schülke; J. Frisch, M. Schülke)

NHMW Naturhistorisches Museum Wien (H. Schillhammer)

SDEI Senckenberg Deutsches Entomologisches Institut (L. Behne)

cAss author's private collection

cFel private collection Benedikt Feldmann, Münster, Germany

cPüt private collection Andreas Pütz, Eisenhüttenstadt, Germany

cTro private collection Marc Tronquet, Molitgles -Bains, France

cWun private collection Paul Wunderle, Mönchengladbach, Germany

cZan private collection Adriano Zanetti, Verona, Italy
The morphological studies were conducted using a Stemi SV 11 microscope (Zeiss), a Discovery V12 microscope (Zeiss), and a Jenalab compound microscope (Carl Zeiss Jena). The images were created using a digital camera (Nikon Coolpix 995), Axiocam ERc 5s, and Picolay software. The Maps were created using MapCreator 2.0 (primap) software.

Body length was measured from the anterior margin of the mandibles (in resting position) to the abdominal apex, the length of the forebody from the anterior margin of the mandibles to the posterior margin of the elytra, head length from the anterior margin of the clypeus to the posterior constriction of the head, elytral length at the suture from the apex of the scutellum to the posterior margin of the elytra, and the length of the aedeagus from the apex of the ventral process to the base of the aedeagal capsule. The "parameral" side (i.e., the side where the sperm duct enters) is referred to as the ventral, the opposite side as the dorsal aspect. Zoogeographic terminology is primarily based on LATTIN (1967).

\section{The subgenus Baeoglena in the West Palaearctic region}

Diagnosis of subgenus: Species of relatively small size $(2.0-3.5 \mathrm{~mm})$. Whole body with fine and dense punctation. Pronotum large in relation to head (Fig. 40), rather strongly convex in cross-section, broadest at or near posterior angles. Head somewhat resembling that of Myllaena ErICHSON, 1837, i.e., wedge-shaped, somewhat elongated anteriorly, and with conspicuously elongated maxillary palpi (Fig. 60). Antennae (Figs 40, 60) relatively short, gradually incrassate apically, and with distinctly transverse antennomeres V-X. Abdomen (Figs 40,60) wedge-shaped, i.e., gradually narrowed from base to apex. Sternite VIII with weakly pronounced sexual dimorphism.

$\sigma^{\star}$ : posterior margin of sternite VIII convex (not angularly or acutely produced in the middle); median lobe of aedeagus rather slender, often with small crista apicalis (e.g., Figs 1-4); ventral process slender in ventral view, in continental species apically blunt or truncate, rarely acute in lateral view; internal sac with a moderately long apical flagellum, with additional apical spines, and often with an apico-ventral filiform structure ("fs" in Figs 39, 48); paramere much longer than median lobe, with very long, nearly straight, basally more or less strongly dilated, and apically very slender and straight apical lobe, basal portion often with species-specific modifications.

ㅇ: posterior margin of sternite VIII weakly convex; spermatheca (e.g., Fig. 9) of simple (and uniform) shape, without evident modifications. 
Comparative notes: Baeoglena species are best distinguished from representatives of other subgenera with elongated maxillary palpi (e.g., O. lurida, O. recondita) by the characteristic morphology of the median lobe and the paramere of the aedeagus. Among West Palaearctic Oxypoda, the paramere most resembles that of species of the subgenus Deropoda Bernhauer, 1902, from which Baeoglena is distinguished by much finer punctation of the forebody, the shape of the head, a broader and shorter body with a more wedge-shaped abdomen, longer maxillary palpi, a weakly pronounced sexual dimorphism of sternite VIII, and by the internal structures of the aedeagus.

In the material examined, specimens erroneously identified as Baeoglena species, mostly belonged to Oxypoda annularis (MANNERHEIM, 1830), some also to O. lurida, O. recondita, and other Oxypoda species. Misidentified O. annularis were even found among the Baeoglena material in the Bernhauer collection.

Wing polymorphism: Wing development was not examined systematically, but based on samples of all the species studied, O. praecox and O. kuehnelti are apparently wingdimorphic with the hind wings in the brachypterous morph of $O$. praecox of distinctly reduced length and in the submacropterous morph of O. kuehnelti of slightly reduced length. Oxypoda derecta is micropterous. No brachypterous or micropterous specimens were observed in the four remaining species.

Intraspecific variation: The present study revealed that the species of the West Palaearctic region exclusive of the Canary Islands are subject to remarkable intraspecific variation of all the external characters previously used for identification (body size, coloration, punctation, etc.). Intraspecific variation of colour and size is partly clinal. For instance, Iberian populations of $O$. fusina are usually of more or less uniformly reddish coloration, whereas specimens from Corsica are often rather dark, sometimes dark-brown with blackish head. Similarly, material of $O$. rectacia is more or less distinctly bicoloured in much of its range, but may also be mostly reddish in some regions or dark-brown with blackish head (especially in Cyprus).

Identification of species: In view of general uniformity combined with pronounced intraspecific variation, a reliable identification based on external characters is usually not possible. The only exception is the micropterous $O$. derecta from the Caucasus region. Moreover, the spermatheca may be suitable for distinguishing species groups, but is otherwise too uniform and at the same time too variable to be of any significance for an identification at the species level. The only characters allowing a reliable identification are the shape of the median lobe (shape of the ventral process and of the crista apicalis), the shapes of the apical spines and the apico-ventral filiform structure in the internal sac ("fs" in Figs 39, 48), and in some species also certain modifications (Figs 27, 35) of the basal portion of the paramere. Hence, a reliable identification requires a dissection of the aedeagus and often also an examination of its internal structures.

Diversity and zoogeography: Baeoglena is currently represented in the Palaearctic region by 20 species. Six of them have been reported from the Himalaya and Japan (SCHÜLKe \& SMETANA 2015). Their systematic status and identities are unclear, as they have not yet been revised. However, I have seen true Baeoglena species from Pakistan and Nepal, so that the subgenus is undoubtedly present also in the East Palaearctic. The vast majority of East Palaeartic Oxypoda species has been described by Roberto Pace, who may not have fully embraced Baeoglena, as can be inferred from the fact that he attributed O. giachinoi, the only West Palaearctic representative of the subgenus he described, to the subgenus Mycetodrepa. It can be concluded that an uncertain number of described East Palaearctic Baeoglena species may currently be assigned to other subgenera.

Fourteen species are at present known from the West Palaearctic region, seven of them endemic to the Canary islands Tenerife and La Gomera, and the remainder distributed in Europe, Asia minor, the Middle East, and the Caucasus region. Except for the micropterous $O$. derecta, all of the latter seven species are rather widespread. Baeoglena species are unknown from North Africa, except for one female-based record of uncertain identity from Morocco.

Remarkably, the distributions of the seven continental species are mostly allo- or parapatric with generally very little overlap (Map 1). Such zones of narrow overlap are the south of mainland Greece (O. nova and O. rectacia) and Bulgaria (O. nova and $O$. praecox). The only exception from this rule is North Turkey where both O. caucasica and O. nova occur. It seems noteworthy, however, that (1) O. caucasica is much rarer in Northwest Anatolia and more common in Northeast Anatolia than O. nova, that (2) O. caucasica and O. nova were, on rare occasions, even found in the same locality, and that (3) O. caucasica and $O$. nova belong to different lineages.

From a zoogeographic perspective, the most interesting region is the southern Balkans (Maps 1, 5). The distributions of the three species present in this region (O. nova, O. kuehnelti, O. rectacia) are both remarkably parapatric and, especially in the case of O. nova, conspicuously discontinuous. The only plausible explanation for such a distribution pattern is interspecific competition among these species. Another such region with distributions parapatrically bordering on each other is South Italy, where the known distribution of O. nova is confined to Calabria and in the north limited by that of O. fusina. Here the range of O. nova is also remarkably discontinuous, with the geographically nearest populations in Croatia and the southern Balkans. Except for rare cases in North Turkey (O. nova and O. caucasica; see above), a syntopic occurrence of two or more Baeoglena species was never observed. This even applies to Oros Kalli- 
dromo (South Greece: Fthiotis), from where both O. nova and $O$. rectacia were recorded, but never in the same sample. To my knowledge, a similar example of interspecific competition clearly determining distributions in Staphylinidae as been observed only in the genus Othius STEPHENS, 1829, with closely related species outcompeting each other in parts of their ranges (O. lapidicola MärkEL \& Kiesenwetter, 1848 - O. subuliformis Stephens, 1833 - O. crassus Motschulsky, 1858; O. subuliformis - O. wunderlei Assing, 1997 - O. brevipennis KraAtz, 1857) (Assing 2003). What Othius and Baeoglean species have in common, too, is that they inhabit the leaf litter layer and often occur at high densities.

The examined collections contained only little material of Baeoglena from France and none from North Italy. Material from these regions would be required to determine the western and southwestern limit of O. praecox, the northern distribution limit of $O$. fusina, and to clarify whether or not the mainland distribution of O. fusina is discontinuous, i.e., if the South Italian and Iberian populations are really separated by such a remarkable distance.

Intrasubgeneric affiliations: Based on the morphology of the aedeagus and of the spermatheca, the seven Baeoglena species of the West Palaearctic region exclusive of the Canary Islands belong to two lineages. The O. caucasica lineage includes two species (O. caucasica, $O$. derecta) and is characterized by a long crista apicalis of the aedeagus (Figs 55, 61-62), an internal sac of the aedeagus (Figs 55, 61-62, 64) with a basally weakly curved and weakly sclerotized flagellum, and a pair of fork-shaped apical structures, a relatively narrow basal portion of the median lobe of the aedeagus in ventral view (Figs 56,63), a basally rather weakly dilated apical lobe of the paramere, and a minute apical invagination of the spermatheca (Figs 58, 66-67). In the second lineage, which includes the remaining five species, the crista apicalis is very small (e.g., Figs 1-4), the flagellum is basally strongly curved (e.g., Figs 1-4) and often rather strongly sclerotized, the apical internal structures are spine-shaped, the basal portion of the median lobe is larger in ventral view (e.g., Figs 5, 15-16), the apical lobe of the paramere is basally often more strongly dilated, and the apical invagination of the spermatheca is larger and distinctly bell-shaped (e.g., Fig. 9). Among the species of the latter lineage, O. kuehnelti and $O$. rectacia are evidently sister species, as can be inferred from the similar shape of the ventral process of the aedeagus and from the shared presence of a distinct filiform apico-ventral structure in the internal sac of the aedeagus (Figs 39, 48). Oxypoda nova may be the adelphotaxon of the other four species, a conclusion supported by the strongly curved median lobe of the aedeagus, the differently shaped apex of the ventral process both in ventral and in lateral view, the extremely narrow crista basalis, and by the conspicuous modifications of the paramere.

Natural history: All species except O. praecox appear to be ecologically very similar. They inhabit the leaf litter layer of various forest, bush, and shrub habitats at a wide range of altitudes, often at high densities. The habitat requirements of $O$. praecox are less clear: it has

Checklist of the Baeoglena species of the West Palaearctic region

\begin{tabular}{|c|c|}
\hline Species & Distribution \\
\hline brevipennis Wollaston, 1864 & Canary Islands: Tenerife: Anaga \\
\hline caeca Assing, 2005 & Canary Islands: Gomera \\
\hline caucasica BERnHAUER, 1902 & $\begin{array}{l}\text { Caucasus region: North Turkey; Southwest Russia; Georgia; Armenia; Nagorno- } \\
\text { Karabakh; Azerbaijan; Iran }\end{array}$ \\
\hline derecta spec. nov. & Caucasus region: West Caucasus; Northeast Turkey \\
\hline fusina Mulsant \& Rey, 1875 & $\begin{array}{l}\text { West Mediterranean: Portugal; Spain; France: Corsica, southern mainland; Italy: } \\
\text { Sardinia, southern mainland; Morocco? }\end{array}$ \\
\hline gillerforsi ZERCHE, 1996 & Canary Islands: Gomera \\
\hline hernandezi ZERCHE, 1996 & Canary Islands: Tenerife: Anaga \\
\hline kuehnelti SCHEERPELTZ, 1963 & Balkans: Serbia, Albania, Macedonia, Greece \\
\hline meybohmi ZERCHE, 1996 & Canary Islands: Tenerife: Anaga \\
\hline nova BERnHAUER, 1902 & $\begin{array}{l}\text { South Italy; Balkans (Croatia, Bosnia-Herzegovina, Montenegro, Bulgaria); North } \\
\text { Turkey }\end{array}$ \\
\hline praecox ERICHSON, 1839 & $\begin{array}{l}\text { Central, North, and East Europe; Hungaria; Croatia, Bulgaria; Romania?; North } \\
\text { Greece?; Siberia? }\end{array}$ \\
\hline rectacia spec. nov. & $\begin{array}{l}\text { South Greece (including Crete and Aegean Islands); South Turkey; Cyprus; Syria; } \\
\text { Lebanon; Israel }\end{array}$ \\
\hline tenoensis ZERCHE, 1996 & Canary Islands: Tenerife: Teno \\
\hline wunderlei ZERCHE, 1996 & Canary Islands: Gomera \\
\hline
\end{tabular}




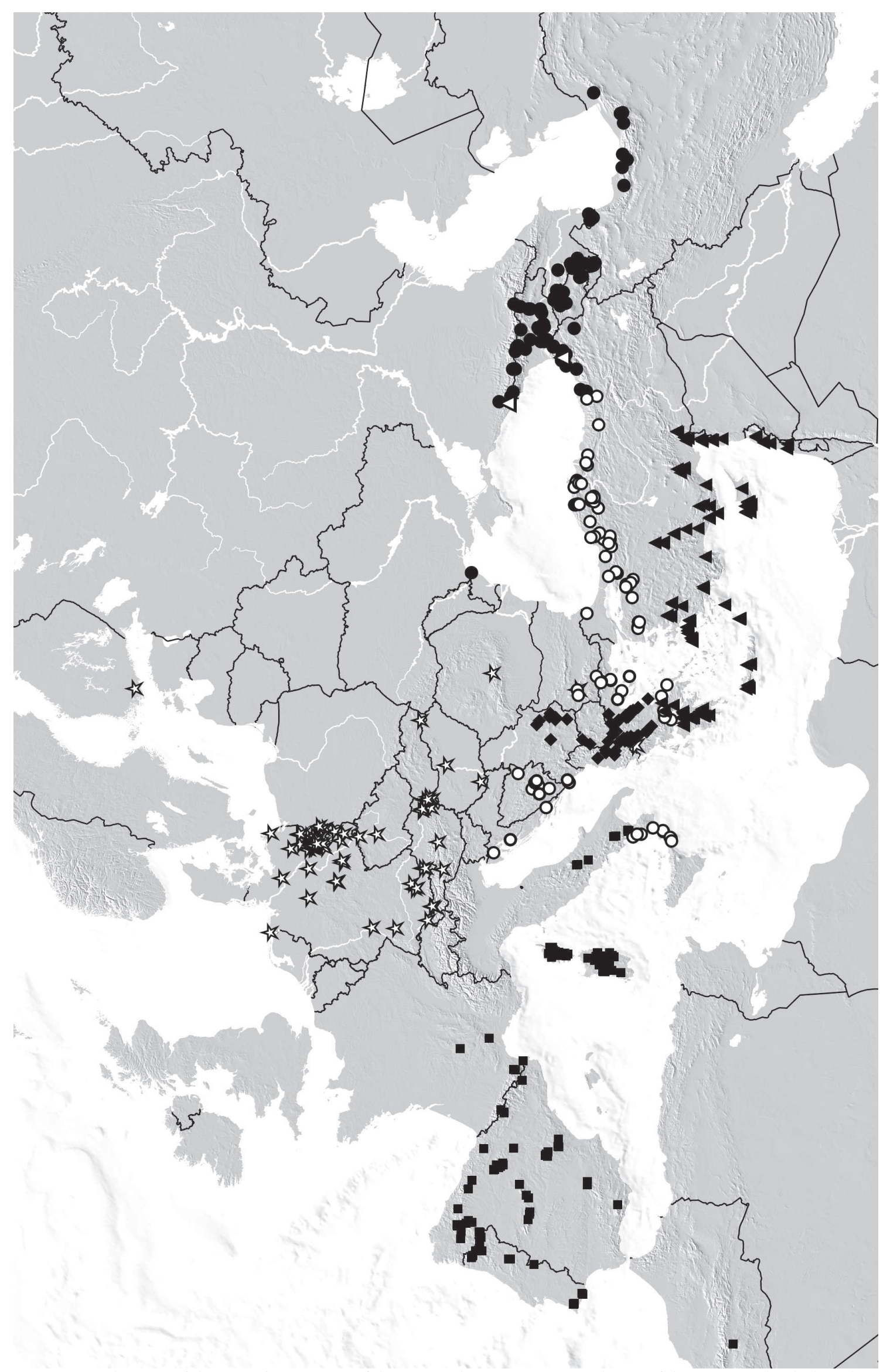

Map 1: Distributions of the Baeoglena species of the West Palaearctic region exclusive of the Canary Islands, based on revised records: Oxypoda praecox (white stars); O. fusina (black squares); O. nova (white circles); O. kuehnelti (black diamonds); O. rectacia (black triangles); O. caucasica (black circles); O. derecta (white triangles). 


\section{Key to the Baeoglena species of the West Palaearctic region exclusive of the Canary Islands}

1. Median lobe of aedeagus with a long crista apicalis, a rather slender basal portion (ventral view), and fork-shaped apical internal structures (Figs 55-56, 62-64). Spermatheca with a minute apical cuticular invagination (Figs 58, 66-67).

- Median lobe of aedeagus with small and short crista apicalis, a broader subcircular basal portion (ventral view), and spine-shaped apical internal structures (e.g., Figs 1-7). Spermatheca with a bell-shaped apical cuticular invagination (e.g., Fig. 9)

2. Hind wings completely reduced; elytra short (Figs 59-60), 0.65-0.70 times as long as pronotum. Median lobe of aedeagus with a narrow crista apicalis (Figs 61-62). West Caucasus; Northeast Anatolia (Maps 1, 7).

derecta

- Hind wings present; elytra at least approximately 0.8 times as long as pronotum. Median lobe of aedeagus with broad crista apicalis (Fig. 55). Widespread in the Caucasus region from Northwest Anatolia to North Iran (Maps 1, 7).

caucasica

3. Ventral process (Figs 23-24) of aedeagus very slender and strongly curved, apically acute in lateral view and concave in ventral view (Fig. 26); basal portion of paramere (Fig. 27) with pronounced modifications (dark structure of distinctive shape). South Italy; Balkans; North Turkey (Maps 1, 5). nova

- Ventral process of aedeagus nearly straight, apically convex or truncate in lateral view and convex or truncate in ventral view; basal portion of paramere without evident modifications or with dark structure of different shape.

4. Ventral process of aedeagus apically not truncate, but more or less convex in lateral view (Figs 10-14, 17-18). Atlanto-Mediterranean: Iberian Peninsula; South France; Corsica; Sardinia; South Italy (Maps 1, 4). fusina

- Ventral process of aedeagus apically truncate.

5. Aedeagus (Figs 29-32) larger; median lobe at least approximately $0.40 \mathrm{~mm}$ long; filiform apico-ventral structure ("fs" in Fig. 39) in internal sac of aedeagus curved (Figs 37-39); basal portion of paramere with circular to trapezoid dark structure ("ds" in Fig. 35). South Balkans: Albania; Serbia; Macedonia; mainland Greece (Maps 1, 5).

kuehnelti

- Aedeagus smaller; median lobe $0.30-0.35 \mathrm{~mm}$ long. Basal portion of paramere without evident modifications. ...

6

6. Aedeagus subapically more distinctly dilated in ventral view (Figs 47, 50), with straight filiform apico-ventral structure ("fs" in Figs 48-49) and with longer apical spines in internal sac (Fig. 48-49). Mostly bicoloured or of dark coloration and with longer elytra. Southern East Mediterranean, from the Pelopónnisos across the Aegean Islands and South Turkey eastwards to the Middle East (Maps 1, 6). rectacia

- Aedeagus subapically less dilated in ventral view (Fig. 5), without filiform apico-ventral structure and with shorter apical spines in internal sac (Figs 6-7). Usually pale-coloured species with generally shorter elytra. Central and North, and East Europe; Balkans; Siberia? (Maps 1-3). praecox

been found in a variety of both forested and unforested habitats, particularly on sandy soils, on islands, and near the coast (see the species section on $O$. praecox below). Adult beetles can be found throughout the year. Teneral specimens were observed in summer.

\section{The Baeoglena species of the West Palaearctic region exclusive of the Canary Islands}

Since external characters are of little use for taxonomic purposes (see comments on identification and intraspecific variation above), the diagnoses given in the species sections below focus on the male sexual characters.
Except for records of O. praecox from North, Central, and East Europe, as well as from Siberia, all previous literature records of other species and from other regions are unreliable and consequently neglected.

\section{Oxypoda (Baeoglena) praecox ERICHson, 1839 (Figs 1-9, Maps 1-3)}

Oxypoda praecox Erichson, 1839: $148 \mathrm{f}$.

Type material examined: Holotype + : “Austria. Schüpp / praecox Er. / 5535 / Syntypus Oxypoda praecox Erichson, 1839, labelled by MNHUB 2018 / Syntypus o Oxypoda praecox Erichson, rev. V. Assing 2018” (MNB). 


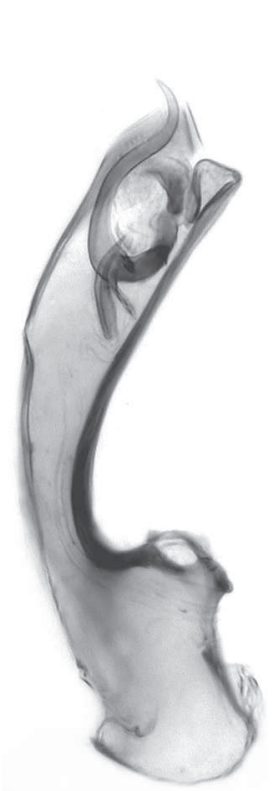

1

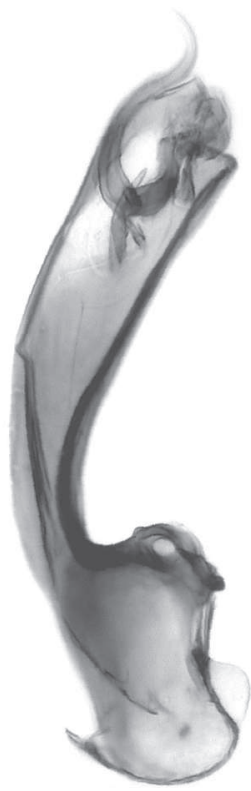

2

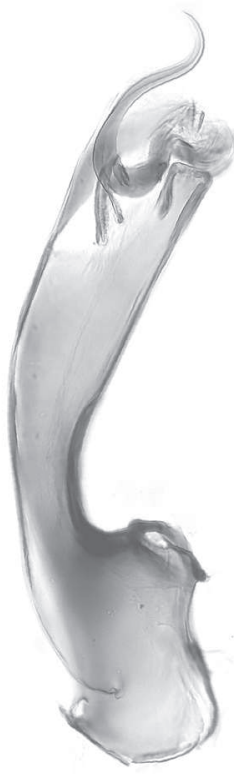

3

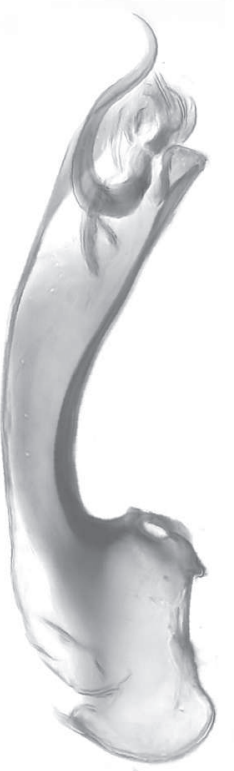

4

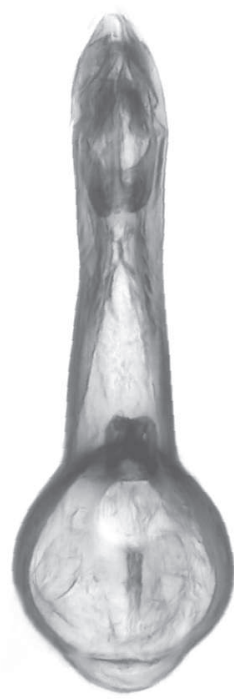

5

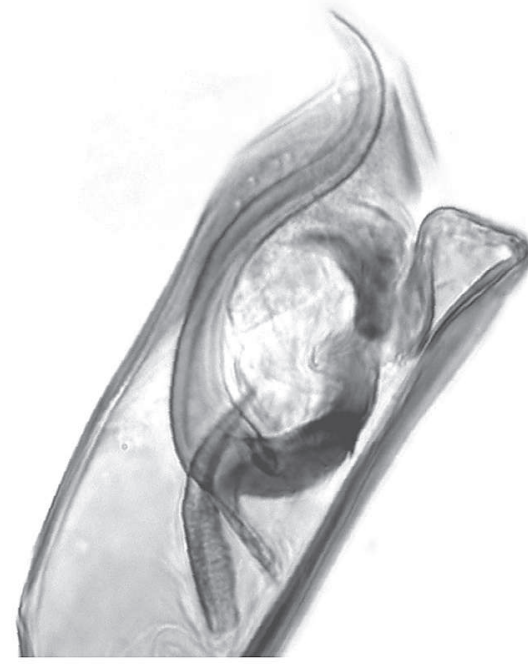

6

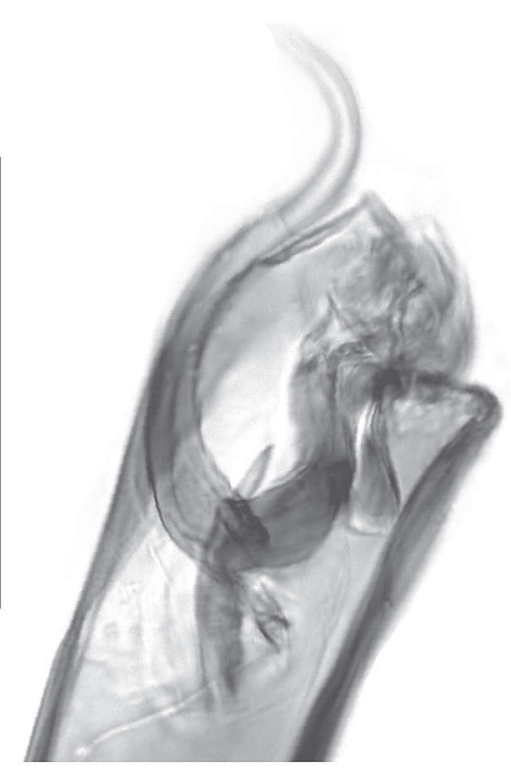

7

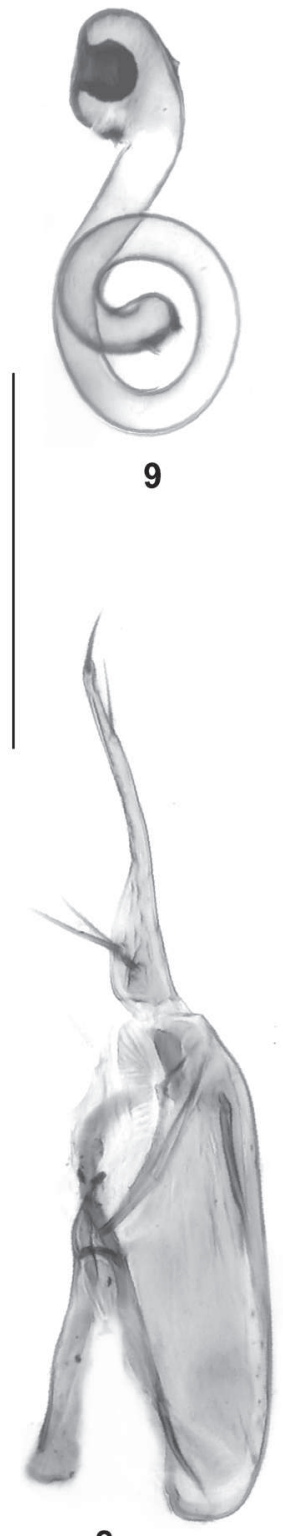

8

Figs 1-9: Oxypoda praecox from Germany (1-2, 5-9), Bulgaria (3), and Slovakia (4): median lobe of aedeagus in lateral and in ventral view (1-5); apex of median lobe of aedeagus in lateral view (6-7); paramere (8); spermatheca (9). Scale bars: 1-5, 8-9: $0.2 \mathrm{~mm} ; 6-7: 0.1 \mathrm{~mm}$

Comment: The original description is based on an unspecified number of syntypes from "Austria" (ERICHSON 1839). Only a single female syntype was located in the historical collection of MNB. The specimen is conspecific with the previous interpretation of this species.

Oxypoda praecox previously had two junior synonyms, which are now synonyms of $O$. fusina. For details see the comment in the section on O. fusina.

Material examined: Finland: 2 exs., Lohja $\left[60^{\circ} 14^{\prime} \mathrm{N}\right.$, $23^{\circ} 43^{\prime} \mathrm{E}$ ], leg. Sahlberg (NHMW).

Germany: Schleswig-Holstein: 1 ex., Mölln, 14.IX.1960, leg. Benick (MNB). Niedersachsen: $1 \sigma^{\star}$, Hannover, fallow, pitfall trap, 30.VI.1986, leg. Assing (cAss); 1 + , Memmert [island], wood debris near compost, 24.V.1995, leg. Rose (cAss). Hessen: 10 , 1 ex., between Bensheim and Heppenheim, NSG "Alte Tongrube", 19.IX.2004, leg. Hetzel (cFel). Baden-Württemberg: 1 ơ, Calw, Ostelsheim, 6.VI.1986, leg. Saure (MNB). Bayern: $10^{\star}$, Landkreis Traunstein, Tittmoning, floodplain of Salzach river, 5.X.2017, leg. Wolf (MNB); 1 ex., Dachau, 26.II.2016 (SDEI); 1 ex., Freising, leg. Scriba (NHMW); 1 ex., München, 20.VIII.1909, leg. Ihssen (MNB); 1 ex., München, 24.VIII.1931, leg. Ihssen (MNB); 1 ex., Lenggries (MNB). Mecklenburg-Vorpommern: 1 ex., SE-Rügen, Neureddewitz, 13.VIII.1974 (cWun); 1 ex., Sassnitz, coast, 17.III.1982, leg. Stöckel 
(MNB); 1 ex., Waren env., NSG „Ostufer der Müritz“, Feldherrenhügel, dry grassland, 14.V.1980, leg. Uhlig \& Zerche (MNB); 1 ex., "Mecklenburg", leg. Konow (NHMW). Sachsen-Anhalt: $3 \sigma^{\top} \sigma^{\top}, 1$, Stendal, Badingen, 24.VI.1993, leg. Sprick (cAss). Berlin/Brandenburg: 1 ex., N-Lausitz, Cottbus env., Tagebau, pitfall trap, 17.X.2002, leg. Steinwarz (cFel); 1 ex., Uckermark, NSG Eulenberge E Kaakstedt, $53^{\circ} 11^{\prime} \mathrm{N}, 13^{\circ} 48^{\prime} \mathrm{E}, 50 \mathrm{~m}$, litter and reed sifted, 9.VI.2013, leg. Schülke (MNB); 1 ex., Landkreis Oder-Spree, Naturpark Schlaubetal, Forst Siehdichum, $52^{\circ} 10^{\prime} \mathrm{N}, 14^{\circ} 27^{\prime} \mathrm{E}$, oak and beech forest, litter sifted, 19.VI.2013, leg. Schülke (MNB); 1 ex., Landkreis Barnim, $1 \mathrm{~km}$ NW Tranpe, $52^{\circ} 47^{\prime} \mathrm{N}, 13^{\circ} 49^{\prime} \mathrm{E}$, moist alder litter sifted, 25.IV.2010, leg. Schülke (MNB); 1 ex., Landkreis Märkisch Oderland, S Zinndorf, sandy field margin and gravel pit, 9.V.2002, leg. Wrase (MNB); 2 exs., Landkreis Havelland, ponds N Linum, sifted, 1.V.1998, leg. Schülke (MNB); 3 exs., Landkreis Oberhavel, Seilershof, Carex, 2.III.1997, leg. Schülke (MNB); 1 ex., same data, but 4.IV.1999, leg. Schülke (MNB); 1 i, 1 ex., Dubrower Forst, sifted, IV.1911, leg. Ude (MNB, cAss); 6 exs., Berlin, Reinickendorf, OT Tegel, Tegeler Fließ am Titusweg, $52^{\circ} 36^{\prime} \mathrm{N}, 13^{\circ} 17^{\prime} \mathrm{E}$, stream bank, dead wood and litter of Salix and Populus sifted, 19.V.2005, leg. Schülke (MNB, cAss); 2 exs., Berlin, Pankow, Tegeler Fließ, 52 $39^{\circ} \mathrm{N}$, $13^{\circ} 24^{\prime} \mathrm{E}$, bank of ditch, sifted, 3.V.2006, leg. Schülke (MNB); 1 ex., Berlin, Pankow, Arkenberge, Tegeler Fließ, meadow, twigs and litter beneath birch trees sifted, 14.IX.2006, leg. Schülke (MNB); 1 ex., Berlin-Friedrichsfelde, Schlosspark, leg. Korge (MNB); 1 ex., Bezirk Cottbus, Lauchhammer, 8.VI.1962, leg. Blaschke (cZan); 1 ex., Berlin, X.1889 (NHMW); 1 ex., Berlin, IV.1890 (NHMW); $10^{*}, 1$ ex., Berlin, Grunewald, 6.VI.1975, leg. Heinel (MNB); 10 , same data, but 15.V.1975 (MNB); 1 ex., same data, but 29.V.1975 (MNB); 1 ex., Berlin, Bahngelände Yorckstraße, leg. Korge (MNB); 1 ex., Berlin, Müllkippe Marienfelde, south slope, 15.VII.1971, leg. Korge (MNB); 2 exs., Berlin, Spandau, Teufelsbruch, 26.V.1975 (MNB); 1 ex., Spandau, ash forest, 17.VII.1979, leg. Korge (MNB); 1 ex., Spandauer Forst, leg. Laß (MNB); 1 ex., Forst Bredow, leg. Korge (MNB); 2 exs., Berlin (SDEI); 1 ex., Bredow, 28.VIII.1922 (MNB); 1 ex., Brieselang, leg. Korge (MNB); 1 ex., Berlin env., Nieder Neuendorf, leg. Laß (MNB); 1 ex., Berlin, Müggelsee, 23.IV.1944 (MNB); 1 ex., Berlin (MNB); 1 ex., Mellensee, 22.III.1945 (MNB); 1 ex., Hennigsdorf, 9.VII.1944 (MNB); 1 ex., Finkenkrug (MNB); 1 ex., Schildow, leg. Korge (MNB); 1 ex., Groß Machnow, NSG Weinberg, 30.IV.1986, leg. Behne (SDEI); 2 exs., Groß Machnow, 8.IV.1950, leg. Bischoff (MNB); 2 exs., Frankfurt/Oder, Strausberg, 10.VII.1982, leg. Schulze (SDEI); 1 ex., Kreis Templin, Suckow, 30.IX.1973, leg. Moritz (SDEI); 1 ex., Berlin, Friedrichshagen, Köpenick, V.1994 (SDEI); 2 exs., Truppenübungsplatz Döberitz, 18.V.1993, leg. Korge (MNB); 1 \%, Storkow env., Philadelphia, NSG Salzstelle Philadelphia, straw and mole nest, 24.III.1984, leg. Schülke (MNB); 2 exs., Luckenwalde, Elsthal, III.1919, leg. Delahon (MNB); 1 ex., same data, but III.1920 (MNB); 1 ex., same data, but III.1921 (MNB);
1 ex., Luckenwalde, Lindenberg, IV.1916, leg. Delahon (MNB); 1 ex., Luckenwalde, locality illegible, X.1919, leg. Delahon (MNB); 1 ex., Erkner, 16.IV.1949 (MNB); 2 exs., Erkner, leg. Bischoff (MNB); 1 ex., Lebus, 16.VI.1995, leg. Barndt (MNB); 1 ex., same data, but 29.VI.1995 (MNB). Thüringen: 1 ex., Kyffhäuser, Wüstes Kalktal, 7.IX.1964, leg. Dorn (SDEI); 5 exs., Kyffhäuser, NSG Kattenburg, 29.V.1984, leg. Zerche (SDEI); 1 ex., Bad Frankenhausen, Kosakenberg, leaf litter, 6.IV.1957, leg. Dieckmann (SDEI); 1 ex., Kattenburg, 4.VI.1916, leg. Petry (MNB). Sachsen: 2 ex., Wittichenau, 12.VII.1986, leg. Vogel (cWun); 2 exs., Leipzig, Connewitzer Holz, 24.IV.1946, leg. Dorn (SDEI); 1 ex., same data, but 11.XI.1951 (MNB); 2 exs., same data, but 19.V.1946 (MNB); 1 ex., same data, but 10.XII.1964 (MNB); 1 ex., same data, but 18.II.1950 (MNB); 1 ex., same data, but 24.IV.1946 (MNB); 1 ex., same data, but. 8.XI.1950 (MNB); 1 ex., Leipzig, Seifertshain, 25.III.1978, leg. Linke (SDEI). Locality ambiguous or illegible: 1 ex., Schönhausen, 18.V.1920 (MNB); 1 ex., Straussberg, 2.IV.1949 (MNB); 1 ex., locality illegible, 20.IV.1922 (MNB); 1 ex., Eppendorf (MNB).

Austria: Vorarlberg: $10^{\star}, 1$ 우, W Bregenz, NSG Rohspitz, flooded meadow, 13.V.1999, leg. Wunderle (cWun); 1 , W Bregenz, Speicherwiese, flooded meadow, 14.V.1999, leg. Wunderle (cWun). Tirol: 1 \&, Lechtal, Stanzach, 900-1300 m, car-net, 25.V.1995, leg. Assing (cAss); 3 exs., Stanzach, Lechauen, dry pine forest, 26.V.1995, leg. Puthz (SDEI). Salzburg: 1 ex., Fuschertal, leg. Franz (NHMW). Steiermark: 2 exs., Admont, leg. Franz (NHMW). Wien/Niederösterreich: 11 exs., Stockerau, leg. Bernhauer, Luze, Skalitzky (NHMW, SDEI, cAss); 1 ex., Marchegg, 1.I.1960, leg. Gotz (NHMW); 1 ex., same data, but 28.II.1960 (NHMW); 1 ex., same data, but 22.XI.1959 (NHMW); 1 ex., Moosbrunn (NHMW); 1 ex., Bisamberg [ $\left.48^{\circ} 19^{\prime} \mathrm{N}, 16^{\circ} 21^{\prime} \mathrm{E}\right]$ (NHMW); 9 exs., Langenzersdorf, leg. Bernhauer, Luze (MNB, NHMW); 1 ex., Wolkersdorf, 22.IV.1962, leg. Franz (NHMW); 1 ex., Braunsberg, 6.V.1950, leg. Schubert (NHMW). Burgenland: 11 exs., Zurndorf, leg. Franz (NHMW). Locality not specified or ambiguous: 1 ex., "Styria", leg. Pipitz (NHMW); 1 ex., Don.-Auen, leg. Otto (NHMW); 2 exs., "Austria" (SDEI).

Czech Republic: 1 ex., Praha, leg. Skalitzky (NHMW); 1 ex., Děčín, leg. Reitter (SDEI).

Slovakia: 1 ơ $^{7}, 5$ exs., Trebišov, 19.IV.1997, leg. Smatana (MNB, cAss); 1 ex., Bratislava, leg. Weber (NHMW).

Hungary: 2 exs., Budapest, leg. Kuthy (NHMW); 2 exs., Pécs, leg. Kaufmann (NHMW).

Romania: 1 ㅇ, Cârţişoara [ $\left.45^{\circ} 43^{\prime} \mathrm{N}, 24^{\circ} 35^{\prime} \mathrm{E}\right]$, leg. Reitter (NHMW); 2 exs., "Transsilvania", 1887, leg. Brenske (NHMW).

Croatia: $10^{\top}$, locality not specified, leg. Hampe (SDEI). Bulgaria: 4 exs., Borowez, 1911, leg. Hilf (NHMW).

Greece: 3 우 [brachypterous], Ipiros, $8 \mathrm{~km} \mathrm{~S}$ Aristi, 500 m, 1.V.1973, leg. Löbl (MHNG).

Russia: 1 ค , Komi Respublika, Uchta, 8.V.1973, leg. Schilow (SDEI); 1 \%, Samara, leg. Faust (NHMW); 2 우, "Quellgebiet des Irkut", 1891, leg. Leder (NHMW). 


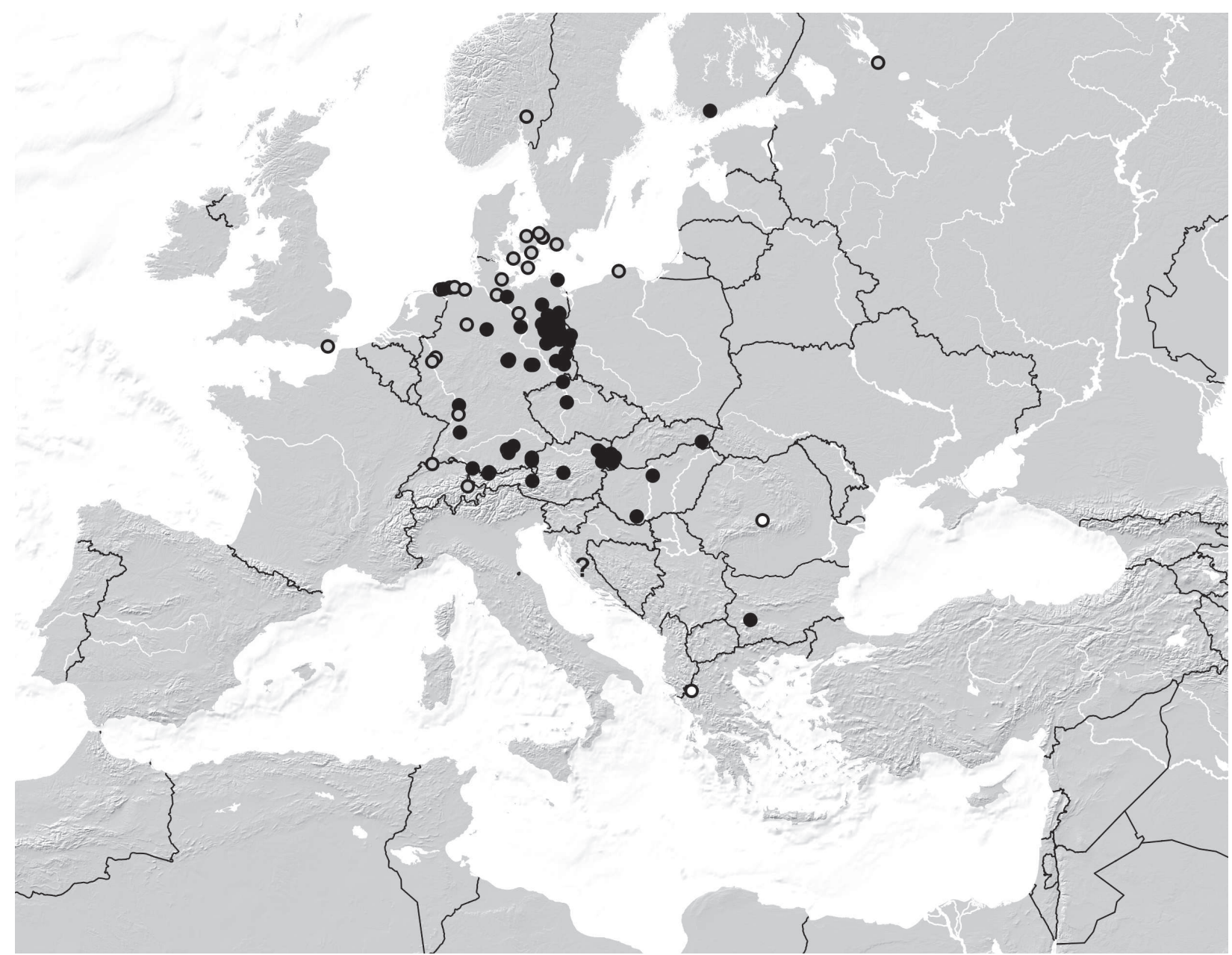

Map 2: Distribution of Oxypoda praecox in Europe, based on revised male-based records (black circles), female-based records (white circles), and selected literature records (grey circles). ?: Record from Croatia without specified locality.

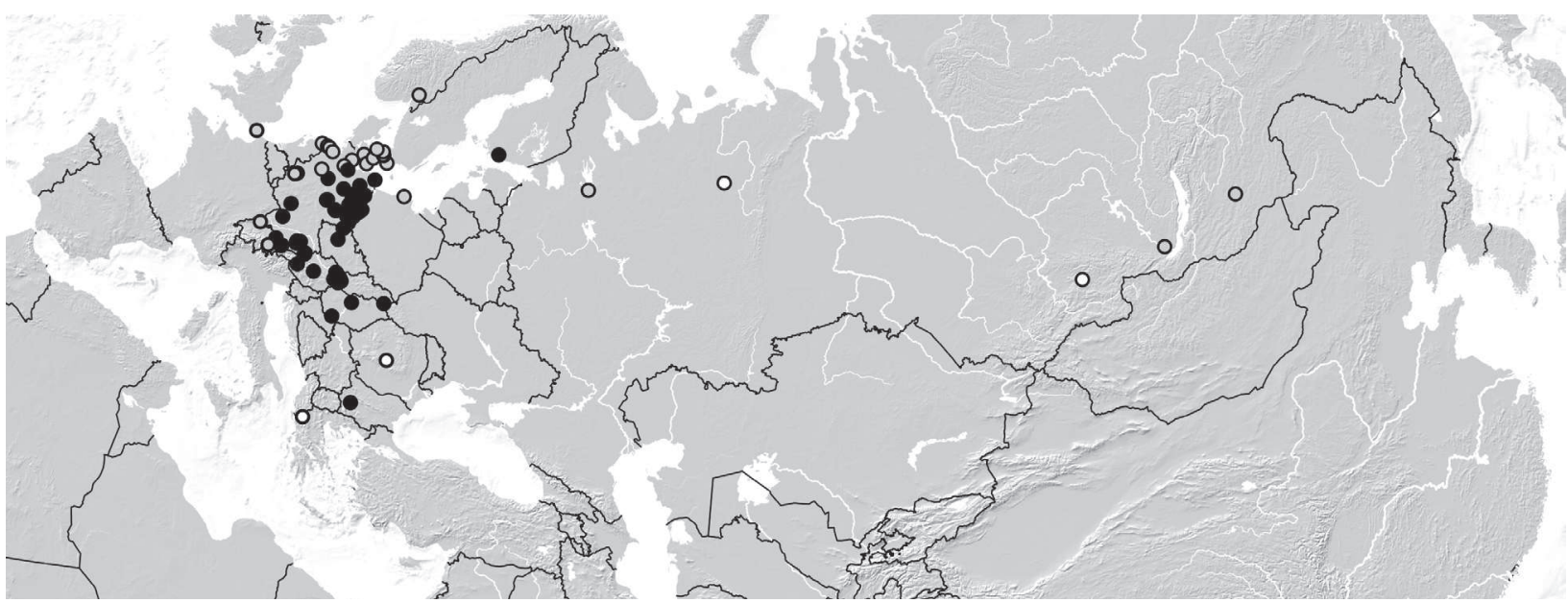

Map 3: Distribution of Oxypoda praecox in the Palaearctic region, based on revised male-based records (black circles), female-based records (white circles), and selected literature records (grey circles).

Diagnosis: Small wing-dimorphic species. Coloration mostly reddish-yellow, more rarely darker or with a darker abdomen.

$0^{*}$ : median lobe of aedeagus (Figs 1-7) $0.31-0.34 \mathrm{~mm}$ long; ventral process apically truncate in lateral view; internal sac with pair of short apical spines, without filiform apico-ventral structure; apical lobe of paramere (Fig. 8) very long, approximately three-fourths as long as basal portion; basal portion of paramere (Fig. 8) without distinct modifications, without dark structure.

: spermatheca (Fig. 9) with bell-shaped apical cuticular invagination. 
Distribution and natural history: In the course of the present revision, male-based records were seen from Finland, Germany, Austria, the Czech Republic, Slovakia, Hungary, and Bulgaria. Additional female-based records from Romania, North Greece, and Siberia are doubtful. In North, Central, and East Europe, the species has - most likely reliably - been reported from Switzerland, Denmark, the Netherlands, Norway, Sweden, Poland, Estonia, Lithuania, and the Russian North and Central European territory (Horion 1967, Schülke \& SMetana 2015). The species is not listed for Great Britain in the Palaearctic Catalogue, but was reported from South England by Williams (1990). For a selection of additional, probably reliable records see BARANOWSKI \& SÖRENSSON (1978), Büche (1994), Grundmann (1991), Hansen (1964), JÖrger (1924), Koch (1978), KöHLER (1988), LOHSE (1967), Rose (2000), and SMOLEŃSKI (1997).

Previous records from other more southern and western regions must be considered highly doubtful and are most likely based on misidentified material. In Central Europe, O. praecox is not uncommon in the northeast, but becoming increasingly rare towards the west. This, the near absence of records from South Europe, and the records from Siberia (if they in fact refer to O. praecox) suggest that the species may be a Siberian faunal element. Previous records from East France (exclusive of Corsica) may refer to O. praecox or O. fusina; those from Southwest France (Tronquet 2006) refer to O.fusina. The revised distribution is illustrated in Maps 1-3.

The species has been collected in a wide range of both moist and dry habitats: in moist debris on shores or banks of standing and running waters, in sand pits, forests, grasslands, urban habitats (dumps, railroad tracks), from leaf litter, nests of mice, rats, moles, and badgers, from ant nests, hollow trees, and straw of field barns (HoRION 1967, Косн 1978, and material examined). According to LoHse (1989), O. praecox is regionally common in pine forests in East Germany. It was also observed to be abundant in coastal dune habitats of islands in Northwest Germany (Rose 2000).

The examined material with dates specified on the labels was collected practically throughout the year, with a maximum in spring: January (1 record); February (3); March (7); April (15); May (21); June (10); July (5); August (4); September (4); October (4); November (3); December (1).

\section{Oxypoda (Baeoglena) fusina Mulsant \& Rey, 1875 (Figs 10-22, Maps 1, 4)}

Oxypoda (Baeoglena) fusina Mulsant \& Rey, 1875: 295.

Oxypoda (Baeoglena) breviuscula Mulsant \& Rey, 1875: 296.

Oxypoda (Baeoglena) corsica Bernhauer, 1899: 423; synonymy by Bernhauer (1902).

Oxypoda (Baeoglena) hispanica FAGEL, 1958: 246 f.; preoccupied; syn. nov.

Oxypoda (Baeoglena) inexpectata FAGEL, 1965: 257 f.; syn. nov.
Comment: The original description of O. fusina is based on an unspecified number of syntypes from "PortoVecchio et Vizzavona, en Corse", that of O. breviuscula on an unspecified number of syntypes from "Ospedale en Corse" (Mulsant \& Rey 1875), and that of O. corsica on an unspecified number of syntypes from "Corsica (Foce)" (Bernhauer 1899). Bernhauer (1902) treated both O. breviuscula and $O$. corsica as junior synonyms of O. fusina. Zerche (1994) examined the type material of O. fusina and O. breviuscula, designated lectotypes, and provided drawings of the genitalia.

FAGEL (1958) based the original description of O. hispanica on a holotype and six paratypes from "Espagne : Casteille, Puerto de Bejar, Collado Franco". Realizing that this name was a junior primary homonym, FAGEL (1965) replaced it with the nomen novum O. inexpectata. Both O. hispanica and O. inexpectata were synonymized with $O$. praecox by TRONQUET (1999). However, based on the present revision, the sole representative of Baeoglena in the Iberian Peninsula is O. fusina, with which the type material of $O$. hispanica is undoubtedly conspecific.

Material examined: Morocco: 1 오 , Haut-Atlas, rte de Tadment, Oughtal, 24.XI.2016, leg. Brustel (cTro).

Portugal: $4 \sigma^{\star} \sigma^{\star}, 3$ 우, Serra da Estrela, S Manteigas, $40^{\circ} 22^{\prime} \mathrm{N}, 7^{\circ} 33^{\prime} \mathrm{W}, 980 \mathrm{~m}$, oak litter, 18.III.2002, leg. Lompe \& Meybohm (cAss); 1 ㅇ, Serra da Estrela, W Manteigas, Penhas Douradas, $40^{\circ} 23^{\prime} \mathrm{N}, 7^{\circ} 34^{\prime} \mathrm{W}$, 1500 m, 19.III.2002, leg. Meybohm (cAss); 2 ㅇ 우, Serra da Estrela, NW Manteigas, $40^{\circ} 26^{\prime} \mathrm{N}, 7^{\circ} 35^{\prime} \mathrm{W}, 1420 \mathrm{~m}$, under stones, 20.III.2002, leg. Meybohm (cAss); $30^{\star} o^{\star}$, 2 우 운, Serra da Estrela, NW Manteigas, $40^{\circ} 26^{\prime} \mathrm{N}$, $7^{\circ} 35^{\prime} \mathrm{W}, 1440 \mathrm{~m}$, birch forest with Genista undergrowth, 20.III.2002, leg. Meybohm (cAss); $10^{\top}, 3$ 우 우, Braganca district, Serra de Bornes, $41^{\circ} 47^{\prime} \mathrm{N}, 7^{\circ} 01^{\prime} \mathrm{W}, 830 \mathrm{~m}$, stream valley, Rubus, 20.III.2002, leg. Meybohm (cAss); 2 우 우 Serra de Montezinho, Montezinho, $41^{\circ} 56^{\prime} \mathrm{N}, 6^{\circ} 46^{\prime} \mathrm{W}$, 1030 m, sifted, 21.III.2002, leg. Meybohm (cAss); $10^{\circ}$, Serra de Montezinho, Rio Sabor, 41 ${ }^{\circ} 54^{\prime} \mathrm{N}, 6^{\circ} 47^{\prime} \mathrm{W}, 900 \mathrm{~m}$, poplar litter sifted, 21.III.2002, leg. Meybohm (cAss); $1 \sigma^{\top}$, Tronco, E Chjaves, $41^{\circ} 46^{\prime} \mathrm{N}, 7^{\circ} 18^{\prime} \mathrm{W}$, meadow, sifted, 22.III.2002, leg. Meybohm (cAss); $20^{\star} o^{\star}$, SW Montalegre, Parafita, $41^{\circ} 46^{\prime} \mathrm{N}, 7^{\circ} 50^{\prime} \mathrm{W}, 900 \mathrm{~m}$, leaf litter near stream sifted, 22.III.2002, leg. Meybohm (cAss); 2 ㅇ ㅇ, Serra do Gerês, E Portela de Homem, $41^{\circ} 48^{\prime} \mathrm{N}, 8^{\circ} 07^{\prime} \mathrm{W}, 790 \mathrm{~m}$, oak and laurel litter, 23.III.2002, leg. Lompe (cAss); $20^{\top} \sigma^{\star}$, 2 우 오, Serra do Gerês, SW Portela de Homem, $41^{\circ} 48^{\prime} \mathrm{N}$, $8^{\circ} 09^{\prime} \mathrm{W}, 630 \mathrm{~m}$, oak and laurel litter, 24.III.2002, leg. Lompe \& Meybohm (cAss); 1 o, 2 우 우, Serra do Gerês, 800 m, oak litter, 26.V.1992, leg. Wunderle (cAss); 1 ex., Serra do Gerês, leg. Paulino (NHMW); $50^{\top} o^{\star}, 1$ 우, Serra de S. Marmede, Marvão, 39 $24^{\prime} \mathrm{N}, 7^{\circ} 23^{\prime} \mathrm{W}, 730 \mathrm{~m}$, oak and chestnut litter, 16.III.2002, leg. Meybohm (cAss); $1 \sigma^{\star}, 4$ ㅇ 우, Algarve, Serra de Monchique, Portela Viuva env., 20-21.II.1999, leg. Meybohm (cAss); 1 o $^{*}, 3$ 우 우, Algarve, Serra de Monchique, N Foia, 850 m, 18.II.1999, leg. Meybohm (cAss); $10^{x}$, Algarve, Serra de Monchique, 
NW Picota, 22.II.1999, leg. Meybohm (cAss); 2 우 우, Algarve, Serra de Monchique, NW Foia, $37^{\circ} 19^{\prime} \mathrm{N}$, $8^{\circ} 36^{\prime} \mathrm{W}, 780 \mathrm{~m}$, 15.IV.2002, leg. Meybohm (cAss); 1 ㅇ, Algarve, W Monchique, $37^{\circ} 19^{\prime} \mathrm{N}, 8^{\circ} 34^{\prime} \mathrm{W}, 550 \mathrm{~m}$, Rubus, 15.IV.2002, leg. Meybohm (cAss); 1 ㅇ, Algarve, Monchique, above road to Alferce, $37^{\circ} 19^{\prime} \mathrm{N}, 8^{\circ} 32^{\prime} \mathrm{W}$, 590 m, 10.IV.2002, leg. Meybohm (cAss); 1 ex., Sierra Monchique, leg. Reitter (NHMW); $10^{\star}, 4$ 우 오, Algarve, N Faro, Alportel, $37^{\circ} 11^{\prime} \mathrm{N}, 7^{\circ} 55^{\prime} \mathrm{W}, 370$ m, 9.IV.2001, leg. Meybohm (cAss); 1 o , 10 km W São Brás de Alportel, 400 m, 4.VI.1992, leg. Wunderle (cWun).

Spain: Galicia: 1 ex., Sierra de Ancares, ESE Degrada, $42^{\circ} 49^{\prime} \mathrm{N}, 6^{\circ} 54^{\prime} \mathrm{W}, 1300 \mathrm{~m}$, moist Ilex forest with fern, 10.VII.2004, leg. Assing (cAss); $20^{\star} 0^{\star}, 1$ 우, Sierra de Ancares, Piornedo-Mustallar, 1500 m, 31.V.1996, leg. Starke (cAss); 6 exs., Sierra do Courel, W Visuna, Formigueiros, $42^{\circ} 36^{\prime} \mathrm{N}, 7^{\circ} 07^{\prime} \mathrm{W}, 1540 \mathrm{~m}$, montane birch and oak forest with Luzula undergrowth, sifted, 12.VII.2004, leg. Assing (cAss); 3 exs., Sierra de Ancares, ENE Degrada, $42^{\circ} 50^{\prime} \mathrm{N}$, $6^{\circ} 54^{\prime} \mathrm{W}, 970 \mathrm{~m}$, mixed deciduous forest with very old Castanea sativa, 14.VII.2004, leg. Assing (cAss); 5 exs., Sierra de Ancares, E Degrada, 42 ${ }^{\circ} 50^{\prime} \mathrm{N}, 6^{\circ} 54^{\prime} \mathrm{W}, 1315 \mathrm{~m}$, old mixed forest with Ilex, Betula, and Luzula undergrowth, 16.VII.2004, leg. Assing (cAss); 1 o $^{\star}$, Lugo, Sierra de Ancares, Degrada, Tres Obispos, 1100 m, VI.1997, leg. Balkenohl (cFel); 8 exs., Lugo, Sierra de Ancares, $2.5 \mathrm{~km}$ E Degrada, $42^{\circ} 48^{\prime} \mathrm{N}, 6^{\circ} 54^{\prime} \mathrm{W}$, mixed forest, 12 .VI.2000, leg. Wrase (MNB); 2 exs., Lugo, Ribas Atlas, Monforte, 300 m, 22.V.2003, leg. Valcárcel (MNB); 1 ex., Lugo, Parada de Montes, Sierra do Courel, chestnut forest, 20.VI.2000, leg. Valcárcel (MNB). Asturias: $1 \sigma^{\star}$, NE Puerto de Ventana, $43^{\circ} 04^{\prime} \mathrm{N}, 6^{\circ} 00^{\prime} \mathrm{W}, 1585 \mathrm{~m}, 13 . \mathrm{VI} .2000$, leg. Wrase (MNB). Cantabria: 1 ex., Picos de Europa, 10 km SW Reinosa, $42^{\circ} 58^{\prime} \mathrm{N}, 4^{\circ} 14^{\prime} \mathrm{W}, 1220 \mathrm{~m}, \mathrm{~N}$-slope, mixed oak and beech forest, leaf litter and grass roots sifted, 12.VII.2003, leg. Assing (cAss); 8 exs., Picos de Europa, 50 km WNW Aguiar de Campoo, S Pena Prieta, Cardano de Arriba, $42^{\circ} 59^{\prime} \mathrm{N}, 4^{\circ} 45^{\prime} \mathrm{W}, 1500 \mathrm{~m}$, moist N-slope with birch, 13.VII.2003, leg. Assing (cAss). Pais Vasco: 1 ex., 60 km SE Bilbao, Sierra de Urquilla, SE Arantzazu, peak of Aitzgorri, $42^{\circ} 57^{\prime} \mathrm{N}, 2^{\circ} 20^{\prime} \mathrm{W}, 1470 \mathrm{~m}, \mathrm{~N}$-slope, beech forest, 10.VII.2003, leg. Assing (cAss). Aragón: 30 exs., WNW Teruel, Sierra de Albarracín, NE Noguera, 40³0'N, $1^{\circ} 35^{\prime} \mathrm{W}, 1625 \mathrm{~m}$, NE-slope, oak forest, 11.IV.2003, leg. Assing (cAss); 4 exs., WNW Teruel, Sierra de Albarracín, Sierra Alta, below peak, $40^{\circ} 29^{\prime} \mathrm{N}, 1^{\circ} 35^{\prime} \mathrm{W}, 1850 \mathrm{~m}$, Pinus and Vaccinium litter between stones sifted, 11.IV.2003, leg. Assing (cAss); 21 exs., WNW Teruel, Sierra de Albarracín, Orihuela del Tremada, $40^{\circ} 32^{\prime} \mathrm{N}, 1^{\circ} 40^{\prime} \mathrm{W}, 1520 \mathrm{~m}$, oak forest, 12.IV.2003, leg. Assing (cAss); 1 ex., Sierra de Albarracín (NHMW); 110 exs., 50 km ESE Teruel, $10 \mathrm{~km}$ N Rubielos de Mora, 40² $14^{\prime} \mathrm{N}, 0^{\circ} 38^{\prime} \mathrm{W}, 1255 \mathrm{~m}$, oak forest, 13.IV.2003, leg. Assing (cAss); 1 ex., $30 \mathrm{~km}$ $\mathrm{S}$ Teruel, Sierra del Javalambre, NE Javalambre, $40^{\circ} 09^{\prime} \mathrm{N}$, $01^{\circ} 00^{\prime} \mathrm{W}, 1700 \mathrm{~m}$, N-slope with scattered pine trees, 14.IV.2003, leg. Assing (cAss); 2 exs., 30 km S Teruel, Sierra del Javalambre, Javalambre ski resort, $40^{\circ} 07^{\prime} \mathrm{N}$, $01^{\circ} 01^{\prime} \mathrm{W}, 1860 \mathrm{~m}, \mathrm{~N}$-slope with spruce and pine, sifted,
14.IV.2003, leg. Assing (cAss); 2 exs., $30 \mathrm{~km} \mathrm{~S} \mathrm{Teruel,}$ Sierra del Javalambre, Javalambre ski resort, $40^{\circ} 07^{\prime} \mathrm{N}$, $01^{\circ} 01^{\prime} \mathrm{W}, 1840 \mathrm{~m}$, litter and grass beneath pine trees sifted, 14.IV.2003, leg. Assing (cAss); 21 exs., Teruel, Noguera, 1550 m, 20.V.1960, leg. Besuchet (MHNG); 3 exs., Teruel, Griegos, 1500 m, 21.V.1960, leg. Besuchet (MHNG); 2 exs., Sierra de Albarracin, leg. Franz (cAss); $3 o^{\star} o^{\star}$, Zaragoza, Sierra del Moncayo, Agramonte, $1300 \mathrm{~m}$, sifted, 20.V.1999, leg. Feldmann (cFel); $2 \sigma^{\star} \sigma^{\star}, 1$ ㅇ, Torla, $1200 \mathrm{~m}$, 13.VI.1991, leg. Wunderle (cWun); 7 exs., Huesca, Valle de Ordesa, 1350 m, 12.VI.1966, leg. Besuchet (MHNG). La Rioja: 1 ex., Sierra de la Demanda, ca. $15 \mathrm{~km}$ SE Ezcaray, San Lorenzo, 42 ${ }^{\circ} 15^{\prime} \mathrm{N}, 2^{\circ} 59^{\prime} \mathrm{W}, 1640 \mathrm{~m}$, beech forest, litter and moss sifted, 13.X.2003, leg. Assing (cAss). Castilla-León: 16 exs., Sierra de la Demanda, ca. $40 \mathrm{~km}$ ESE Burgos, S Valmala, Trigaza, $42^{\circ} 16^{\prime} \mathrm{N}, 3^{\circ} 15^{\prime} \mathrm{W}$, $1720 \mathrm{~m}$, beech forest, 12.X.2003, leg. Assing (cAss); 1 ex., Sierra de la Demanda, ca. $40 \mathrm{~km}$ ESE Burgos, S Valmala, Trigaza, $42^{\circ} 17^{\prime} \mathrm{N}, 3^{\circ} 16^{\prime} \mathrm{W}, 1540 \mathrm{~m}$, beech forest with Vaccinium, Juniperus, and grass, 12.X.2003, leg. Assing (cAss); 3 exs., Sierra de la Demanda, ca. 40 km E Burgos, NE Valmala, Rábanos, $42^{\circ} 18^{\prime} \mathrm{N}, 3^{\circ} 16^{\prime} \mathrm{W}, 1190 \mathrm{~m}$, oak forest with grass, 12.X.2003, leg. Assing (cAss); 34 exs., Sierra de la Demanda, Sierra de Urbión, Sierra de Freguela, Peña Negra, $42^{\circ} 03^{\prime} \mathrm{N}, 2^{\circ} 46^{\prime} \mathrm{W}, 1750-1950 \mathrm{~m}$, pine forest, 15.X.2003, leg. Assing (cAss); 5 exs., Sierra de la Demanda, E Neila, Cabeza Herrera, $42^{\circ} 05^{\prime} \mathrm{N}, 2^{\circ} 58^{\prime} \mathrm{W}$, $1580 \mathrm{~m}$, E-slope, mixed oak, beech, and pine forest, 16.X.2003, leg. Assing (cAss); 3 exs., Sierra de la Demanda, N Huerta de Arriba, $42^{\circ} 08^{\prime} \mathrm{N}, 3^{\circ} 05^{\prime} \mathrm{W}, 1230 \mathrm{~m}$, oak forest, 16.X.2003, leg. Assing (cAss); 14 exs., 25 km SE Ponferrada, Montes de Leon, $42^{\circ} 30^{\prime} \mathrm{N}, 6^{\circ} 24^{\prime} \mathrm{W}, 1465 \mathrm{~m}$, oak forest with Erica undergrowth, 21.VI.2002, leg. Assing (cAss); 1 ex., ca. $40 \mathrm{~km}$ SE Ponferrada, Sierra del Teleno, El Teleno, $42^{\circ} 21^{\prime} \mathrm{N}, 6^{\circ} 25^{\prime} \mathrm{W}, 1965 \mathrm{~m}$, birch litter and grass in the shade of rocks sifted, 22.VI.2003, leg. Assing (cAss); 1 ex., Sierra de Ancares, $3 \mathrm{~km}$ E Piornedo, $42^{\circ} 52^{\prime} \mathrm{N}$, $6^{\circ} 50^{\prime} \mathrm{W}, 1200 \mathrm{~m}$, N-slope, stream valley with birch, oak, and Luzula undergrowth, deep litter sifted, 15.VII.2004, leg. Assing (cAss); 1 ex., Sierra de Gredos, ca. $60 \mathrm{~km} \mathrm{SW}$ Avila, ca. $5 \mathrm{~km} \mathrm{~S}$ Hoyos del Espino, $40^{\circ} 18^{\prime} \mathrm{N}, 5^{\circ} 14^{\prime} \mathrm{W}$, $1470 \mathrm{~m}$, stony grassland in stream valley, under stones, 24.III.2007, leg. Assing (cAss); 13 exs., Sierra de Gredos, ca. $60 \mathrm{~km}$ SW Avila, Puerto de Serranillos, $40^{\circ} 20^{\prime} \mathrm{N}$, $4^{\circ} 56^{\prime} \mathrm{W}, 1420 \mathrm{~m}$, narrow stream valley with shrubs and Rubus, sifted from deep litter and soil, floated, 24.III.2007, leg. Assing (cAss); 2 exs., Sierra de Gredos, ca. 60 km SSW Avila, ca. $10 \mathrm{~km}$ SSW Burgohondo, $40^{\circ} 21^{\prime} \mathrm{N}, 04^{\circ} 49^{\prime} \mathrm{W}$, $1250 \mathrm{~m}$, stream valley, floated, 25.III.2007, leg. Assing (cAss); $2 \sigma^{\top} \sigma^{\star}, 1$ 우, Soria, Sierra de Urbión, below Laguna Negra, 1600 m, 21.V.1999, leg. Feldmann (cFel); 15 exs., Ponferrada, leg. Paganetti (FMNH, MNB, NHMW). Madrid: 7 exs., Sierra Guadarrama, B. Escorial, leg. Franz (NHMW); 1 ex., Navacerrada env., VII.1957, leg. Comellini (MHNG); 11 exs., Fuente Fria, 1700 m, 13.IV.1960, leg. Besuchet (MHNG). Cataluña: $2 \sigma^{\top} o^{*}, 2$ ㅇ ㅇ, Barcelona, Sierra del Cadi, Col de Pal, 1800 m, 31.III.1994, leg. Assing (cAss). Castilla-La Mancha: 32 exs., SW Albacete, 
Sierra de Alcaraz, $20 \mathrm{~km} \mathrm{~S} \mathrm{Alcaraz,} 38^{\circ} 33^{\prime} \mathrm{N}, 02^{\circ} 30^{\prime} \mathrm{W}$, $1110 \mathrm{~m}, \mathrm{~N}$-slope with grass, under stones, 7.IV.2003, leg. Assing (cAss); 7 exs., Sierra de Alcaraz, ca. 15 km NNE Riópar, $38^{\circ} 35^{\prime} \mathrm{N}, 02^{\circ} 21^{\prime} \mathrm{W}, 1125 \mathrm{~m}$, deciduous trees near stream, 10.IV.2003, leg. Assing (cAss). Andalucía: 1 q [teneral], Granada, Sierra Nevada, Puerto de la Ragua, 2000 m, 31.VII.2002, leg. Pütz (cAss).

France: Pyrénées-Orientales: $1 \sigma^{*}$, forêt de Boucheville, 750-950 m, 27.II.2001, leg. Tronquet (cTro); 3 o $^{7}$, Molitg, pic del Roussillou, 1280 m, 30.IV.1999, leg. Tronquet (cTro); 1 q, Gèdre, le Maillet, 1800 m, 25.V.2015, leg. Perez (cTro); 1 \%, Albères, Pic Neoulos, $1200 \mathrm{~m}$, 23.V.1996, leg. Tronquet (cTro). Massif Central: $1 o^{\text {T}}$, Auvergne, Cantal, Plomb du Cantal, $1850 \mathrm{~m}, 20-21$. VI.2000, leg. Tronquet (cTro). Gard: 1 , Col de Triballe, $44^{\circ} 02^{\prime} \mathrm{N}, 3^{\circ} 43^{\prime} \mathrm{E}, 600 \mathrm{~m}, 18 . V I .2000$, leg. Tronquet (cTro). Corse: $3 \sigma^{\star} \sigma^{\star}, 4$ 우 우, Monte San Petrone, $1100 \mathrm{~m}$, sifted, 7.IV.1990, leg. Assing (cAss); 1 o $^{\star}, 1$ ex., 30 km S Corte, Col de Sorba, 1300 m, 10.IV.1990, leg. Assing \& Wunderle (cAss, cWun); 1 ㅇ, $30 \mathrm{~km}$ W Corte, Col de Vergio, $1600 \mathrm{~m}$, pine litter sifted, 9.IV.1990, leg. Assing (cAss); 1 ex., Col de Vergio, 1500 m, 9.IV.1990, leg. Wunderle (cWun); 1 ex., Col de Vergio, 1500 m, 30.IV.2001, leg. Wolf (MNB); 14 exs., Valle di Restonica, 800-1000 m, 6-13.IV.1990, leg. Wunderle (cWun); 1 †, $20 \mathrm{~km} \mathrm{SE} \mathrm{Corte,}$ Erbajolo env., stream bank, 12.IV.1990, leg. Assing (cAss); 1 ㅇ, 1 ex., $S$ Aullene, near intersection to Zerubia, $750 \mathrm{~m}$, 7.V.2001, leg. Wolf (MNB, cAss); 1 o , 3 exs., Haute-Corse, Castagniccia, between Campania and Valle de Alesani, 800 m, 10.V.2001, leg. Wolf (MNB, cAss); 1 o $^{\star}, 8$ exs., Haute-Corse, Col de Vizzavona, PylO. Telekom, 1200 m, 3.V.2001, leg. Wolf (MNB, cAss); 2 exs., Col de Vizzavona, Majs, $1100 \mathrm{~m}, 2 . \mathrm{V} .2001$, leg. Wolf (MNB); 1 ex., W Asco, Haute Asco, 850 m, 9.V.2001, leg. Wolf (MNB); 5 exs., Castagniccia, 1150 m, 7.IV.1990, leg. Wunderle (cWun, cZan); 8 exs., Colle di Verde, 8.VII.1992, leg. Zanetti (cZan); 5 exs., Val d'Ese, 1600 m, 9.VII.1991, leg. Zanetti (cZan); 12 ex., Val d'Ese, Bastelica, 1600 m, 7.VIII.1988, leg. Zanetti (cZan); 1 ex., For. Vizzavona, 8.VII.1991, leg. Zanetti (cZan); 1 ex., Haute Asco, 1500 m, 28.VII.1976, leg. Sette (cZan); 1 ex., Haute-Corse, Fontane de Padula, $3 \mathrm{~km}$ W Vezzani, $42^{\circ} 11^{\prime} \mathrm{N}, 9^{\circ} 13^{\prime} \mathrm{E}, 900 \mathrm{~m}$, wet moss beneath pine trees, 29.VII.1998, leg. Zerche \& Ruch (SDEI); 7 exs., Forêt de l'Ospedale, W reservoir, $800 \mathrm{~m}$, 25.VII.1994, leg. Zerche (SDEI); 4 exs., Col de Bavella, 1240 m, 25.VII.1994, leg. Zerche (SDEI); 2 exs., E Bastelica, $42^{\circ} 00^{\prime} \mathrm{N}, 9^{\circ} 07^{\prime} \mathrm{E}, 1550 \mathrm{~m}$, stream bank, 21.VII.1998, leg. Zerche (SDEI); 1 ex., Mt. Renoso, Bergeries de Campannelle SW Ghisoni, 42 $05^{\prime} \mathrm{N}, 9^{\circ} 09^{\prime} \mathrm{E}, 1610$, Alnus viridis litter near stream sifted, leg. Zerche (SDEI); 3 exs., Mt. Renoso, 1905, leg. Leonhard (SDEI); 1 ex., locality not specified, leg. Fauvel (NHMW).

Italy: Sardegna: $1 \mathrm{o}^{\top}, 30 \mathrm{~km}$ W Siniscola, $40^{\circ} 32^{\prime} \mathrm{N}$, $9^{\circ} 20^{\prime} \mathrm{E}, 810 \mathrm{~m}$, moist oak forest with rocks, litter and grass roots sifted, 23.XII.2012, leg. Assing (cAss); 1 ㅇ, $17 \mathrm{~km}$ SW Siniscola, Mont'Albo, 40²9'N, 9³2'E, $610 \mathrm{~m}$, macchia with Quercus ilex, ltter and grass roots sifted, 23.XII.2012, leg. Assing (cAss); 1 으, Monti del Gennar- gentu, Bruncu Spina, $40^{\circ} 03^{\prime} \mathrm{N}, 9^{\circ} 18^{\prime} \mathrm{E}, 1280 \mathrm{~m}$, oak forest, leaf litter near stream and at foot of very old oak trees sifted, 25.XII.2012, leg. Assing (cAss); $10^{\star}, 2$ ㅇ 우, Monti del Gennargentu, $6 \mathrm{~km}$ S Fonni, 4004' N, 9 $15^{\prime} \mathrm{E}, 1100 \mathrm{~m}$, oak forest, litter sifted, 26.XII.2012, leg. Assing (cAss); 4 우 ㅇ, Monti del Gennargentu, $10 \mathrm{~km}$ SE Fonni, $40^{\circ} 05^{\prime} \mathrm{N}$, $9^{\circ} 21^{\prime} \mathrm{E}, 1170 \mathrm{~m}$, oak forest with rocks, litter sifted, 26.XII.2012, leg. Assing (cAss); 3 o $0^{\star}, 3$ 우 우, Monte Arci, $12 \mathrm{~km}$ NE Terralba, Genna Spina, 39² $48^{\prime} \mathrm{N}, 8^{\circ} 45^{\prime} \mathrm{E}, 710 \mathrm{~m}$, oak forest, litter sifted, 27.XII.2012, leg. Assing (cAss); 2 우, $30 \mathrm{~km} \mathrm{~W} \mathrm{Nuoro,} \mathrm{Catena} \mathrm{del} \mathrm{Márghine,} 4 \mathrm{~km}$ N Bolótana, $40^{\circ} 21^{\prime} \mathrm{N}, 8^{\circ} 55^{\prime} \mathrm{E}, 1000 \mathrm{~m}$, oak forest, leaf litter sifted, 30.XII.2012, leg. Assing (cAss); $3 \sigma^{\star} \sigma^{\star}$, Nuoro, Forest di Montes, $40^{\circ} 10^{\prime} \mathrm{N}, 9^{\circ} 21^{\prime} \mathrm{E}, 970 \mathrm{~m}, 6 . \mathrm{IV} .2013$, leg. Meybohm (cAss); $60^{\star} 0^{\star}$, Nuoro, Lanusei, Bosco Selene, $39^{\circ} 52^{\prime} \mathrm{N}, \quad 9^{\circ} 31^{\prime} \mathrm{E}, 900 \mathrm{~m}, 30 . I I I .2013$, leg. Meybohm (cAss); $20^{\star} 0^{\star}, 1$ 우, Pattada (SS), 30.X.1986, leg. Giachino (cAss); 1 o , M. Limbaca, 1000 m, 20.II.2010, leg. Magrini (cAss); 1 ex., Montiferru N Seneghe, $40^{\circ} 07^{\prime} \mathrm{N}, 8^{\circ} 35^{\prime} \mathrm{E}$, 800 m, 25.III.2014, leg. Meybohm (cAss); 2 exs., Nuoro, $20 \mathrm{~km}$ N Oliena, $40^{\circ} 20^{\prime} \mathrm{N}, 9^{\circ} 24^{\prime} \mathrm{E}, 460 \mathrm{~m}$, 3.IV.2014, leg. Meybohm (cAss); 1 ex., Nuoro, 6 km N Lula, Monte

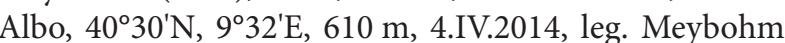
(cAss); 1 ๙ $^{\star}, 5$ exs., M. Limbara (SS), 1000 m, oak forest, 29.V.1995, leg. Angelini (cAss); 4 o $^{\star} 0^{\star}$, Monti del Gennargentu, Punta la Marmora, 40 $01^{\prime} \mathrm{N}, 9^{\circ} 17^{\prime} \mathrm{E}, 1600 \mathrm{~m}$, sifted, 16.V.2005, leg. Hetzel (cFel); 3 exs., Gennargentu, M. d'Iscudo, $1300 \mathrm{~m}, 22 . V .1997$, leg. Zoia \& Polese (cZan); $20^{\star} o^{\star}, 2$ 우 온, Catena d. Marghine, Mt. Palai, $1000 \mathrm{~m}$, leaf litter, 12.X.1989, leg. Wunderle (cWun); 2 exs., L. Bau Mela [approx. $40^{\circ} 00^{\prime} \mathrm{N}, 9^{\circ} 25^{\prime} \mathrm{E}$ ], $850 \mathrm{~m}$, 21.IV.1992, leg. Scheuern (cWun); 5 exs., Lanusei (NU), SS 198 km 89, 2.VII.1984, leg. Torti (cZan); 3 exs., Lanusei (NU), M. Seleni, 900 m, 31.IV.1987, leg. Torti (cZan); 1 ex., Osini (NU), 13.VII.1984, leg. Torti (cZan); 1 ex., Baunei (NU), San Pietro, 23.VII.1986, leg. Torti (cZan); 20 exs., Villagrande Strisaili (NU), 18.IV.1989, leg. Torti (cZan); 2 exs., Villanova Strisaili (NU), SS 389 km 173, 20.IV.1989, leg. Torti (cZan); 2 exs., Monte Ferru [400'스, $8^{\circ} 36^{\prime} \mathrm{E}$ ], leg. Dodero (NHMW); 6 exs., Aritzo, leg. Krüger (NHMW); 2 exs., Sorgono, leg. Krausse (SDEI); 1 ex., Seneghe, leg. Lostia (MNB); 1 ex., Leni, leg. Dodero (FMNH). Abruzzi: $1 \sigma^{*}$, L’Aquila, Rivisondoli, VIII.1925 (FMNH); 2 우 ㅇ, Monte Arazecco, leg. Paganetti (FMNH, SDEI). Campania: 2 우, Lago Matese (CE), $1100 \mathrm{~m}$, beech forest, 17.V.2001, leg. Angelini (cAss). Basilicata: $1 \sigma^{\star}, 3$ 우 우, M. Pierfaone (PZ), 1600-1700 m, 22.VI.1988, leg. Angelini (cAss); $10^{\star}, 1$ ㅇ, Abriola (PZ), La Maddalena, 1400 m, 7-9.VII.19[year not indicated], leg. Angelini (cAss); 4 우 옹 Abriola (PZ), La Maddalena, $1200 \mathrm{~m}$, oak forest, 2.VI.1991, leg. Angelini (cAss); $2 o^{\top} \sigma^{*}$, 2 exs., Pollino, Colle del Dragone, $1600 \mathrm{~m}$, beech forest, 19.V.1991, leg. Angelini (cAss); $20^{\star} 0^{\star}, 3$ 우 우 $14 \mathrm{~km} \mathrm{SSE}$ Mormanno, Monte Pollino, 39 ${ }^{\circ} 56^{\prime} \mathrm{N}, 16^{\circ} 08^{\prime} \mathrm{E}, 10$. V.2002, leg. Wunderle (cWun, cAss); 1 o $^{\star}, 6$ exs., Pollino, Coppola di Paola (PZ), $1400 \mathrm{~m}$, beech forest, 5.VI.1985, leg. Zoia (cZan, cAss); 1 ㅇ, Pollino (PZ), Piani Ruggio, 1500 m, 21.VII.2000, leg. Zanetti (cZan); 2 우 인 Lucania, Piano 


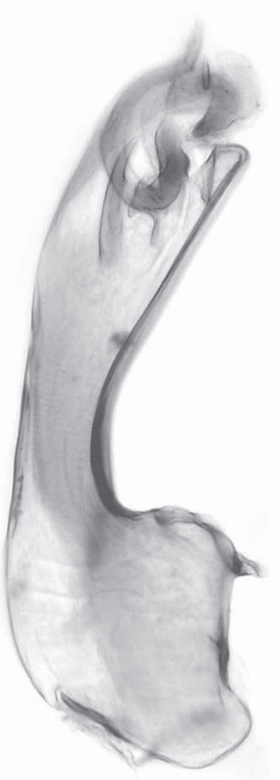

10

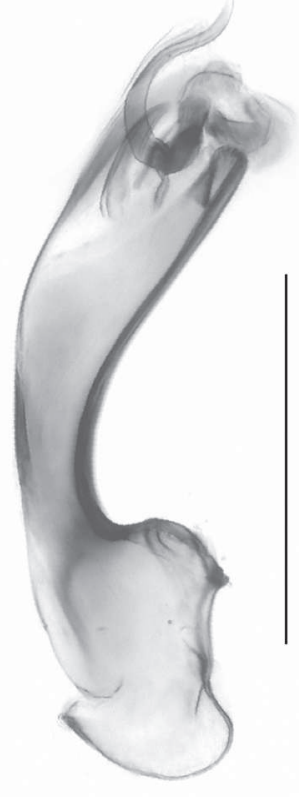

11

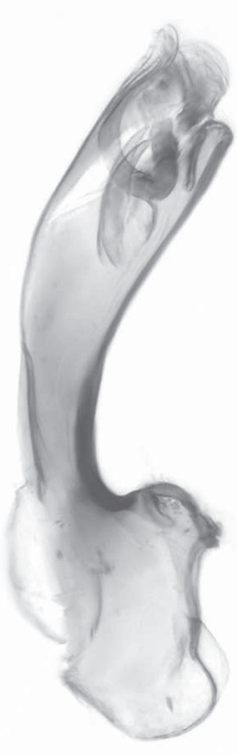

12

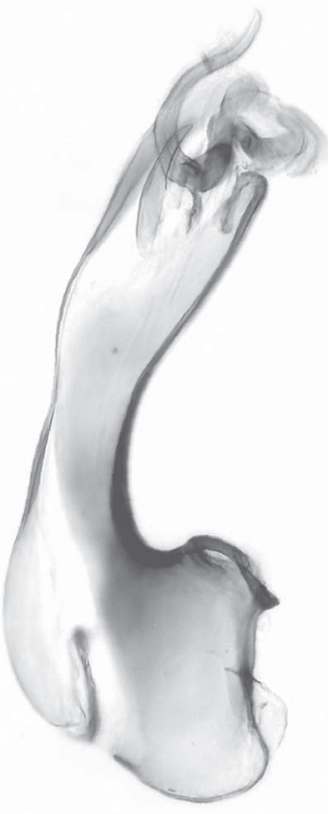

13

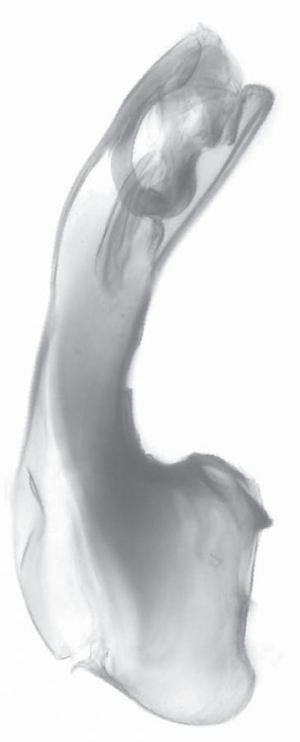

14

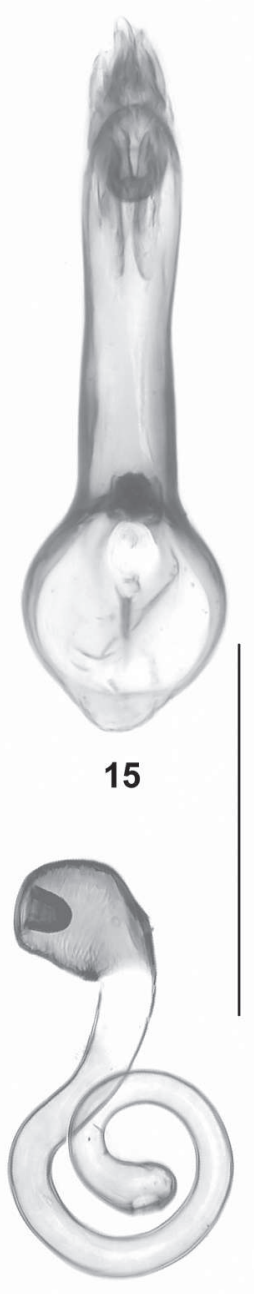

21

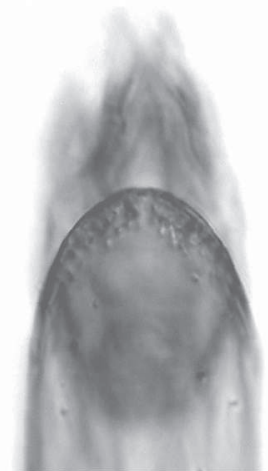

19

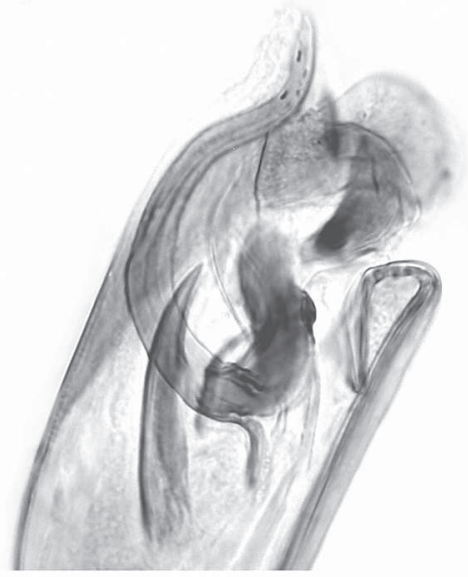

17

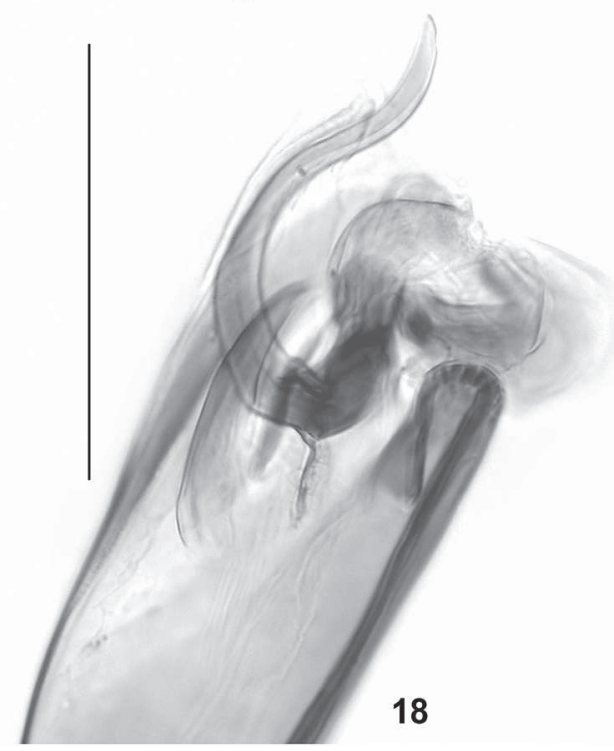

Figs 10-22: Oxypoda fusina from Spain $(10,17)$, Sardinia $(11-12,15,19,22)$, and Corsica $(13-14,16,18,20-21)$ : median lobe of aedeagus in lateral and in ventral view (10-16); apex of median lobe of aedeagus in lateral and in ventral view (17-19); paramere (20); spermatheca (21-22). Scale bars: 10-16, 20-22: 0.2 mm; 17-18: $0.1 \mathrm{~mm}$; 19: $0.05 \mathrm{~mm}$. 
Ruggio, 22.VI.1985, leg. Angelini (cAss). Calabria: $10^{\star}$, Monte Pollino (CS), between Colle Gaudolino and Pollinello, 1750 m, 21.VII.2000, leg. Zanetti (cZan).

Diagnosis: Winged species of variable coloration and size. Populations from the Iberian Penisula and Southwest France usually pale-reddish, material from Corsica often dark-coloured.

$\sigma^{7}$ : median lobe of aedeagus (Figs 10-19) $0.32-0.36 \mathrm{~mm}$ long; ventral process apically not truncate in lateral view and distinctly convex in ventral view; internal sac with very short filiform apico-ventral structure; apical lobe of paramere (Fig. 20) basally rather weakly dilated and very long, approximately three-fourths as long as basal portion; basal portion of paramere (Fig. 20) without distinct modifications, without dark structure.

i : spermatheca (Figs 21-22) of variable shape, with bellshaped apical cuticular invagination.

Distribution and natural history: The distribution of O. fusina (Maps 1,4) is much more restricted than indicated in the Palaearctic Catalogue (Schülke \& SMEtana 2015). The species is common and widespread in the Iberian Peninsula, though very rare in South Spain (only a single specimen known from Andalucía). In addition, it was frequently recorded from Corsica and Sardinia, with some records known also from South France. Based on currently available material, the population in South Italy (Basilicata, Abruzzi, Campania) appears to be rather isolated. The female-based record from Morocco requires confirmation. Previous records of O. praecox from the Pyrenees (Tronquet 2006) refer to O. fusina.

The examined material was mostly sifted from leaf litter and roots in various forest (deciduous, coniferous, mixed) and bush habitats, often in large numbers, but also found in meadows, on stream banks, etc. The altitudes range from 300 to $2000 \mathrm{~m}$. The specimens were found nearly throughout the year: February (6 records); March (17); April (31); May (22); June (15); July (24); August (1); October (9); November (1); December (7). The respective number of records, however, probably reflects the collecting activity more than the actual phenology of the species. Teneral beetles were observed in July.

\section{Oxypoda (Baeoglena) nova Bernhauer, 1902 (Figs 23-28, Maps 1, 5)}

Oxypoda (Baeoglena) nova Bernhauer, 1902: $180 \mathrm{f}$. Oxypoda (Mycetodrepa) giachinoi PACE, 2001: 131 f.; syn. nov.

Type material examined: Lectotype ${ }^{\star}$ [dissected prior to present study], present designation: "nova Brnh, Türkei, Merkl / Chicago NHMus, M. Bernhauer Collection / Lectotypus ox Oxpoda nova Bernhauer / Oxypoda nova Bernhauer, det. V. Assing 2018” (NHMW). Paralectotypes: 1 ex. [abdomen missing]: "Saj / nova Brh. Bosnien, Type.
/ Chicago NHMus, M. Bernhauer Collection” (FMNH); 1 ex. [abdominal apex missing]: "[locality illegible] / nova Brh. Bosnien, Type. / type / ex coll. Skalitzky / Typus Oxypoda nova M. Bernhauer / Oxypoda nova Bernhauer, det. V. Assing 2018” (NHMW); 1 o : "Dalmatia, Reitter. / praecox Er?, nach Smlg Epp / nova Brh Type / Chicago NHMus, M. Bernhauer Collection” (FMNH).

Comment: The original description of O. nova is based on an unspecified number of syntypes from "Bosnien (... Sarajevo ...), Dalmatien und der Türkei” and from "Italien und Spanien" (Bernhauer 1902). Thus, as can be inferred from the revised distributions, the type material includes at least two, possibly even three, or even four species. Four specimens qualifying as syntypes were located in the Bernhauer collection and in the collections of the NHMW. A syntype from Turkey, the only male in fairly good condition among them, is designated as the lectotype.

PACE (2001) described O. giachinoi based on a male holotype and female paratype from "Grecia, nom. Fthiótida, O. Oxià, str. Gardiki-Gramméni Oxià” and erroneously assigned the species to the subgenus Mycetodrepa Thomson, 1859. The illustrations of the aedeagus leave no doubt that $O$. giachino i is conspecific with O. nova.

Material examined: Italy: Calabria: $1{ }^{\star}$, Serra S. Bruno, $6 \mathrm{~km}$ W Mongiana, 1100 m, 21.VI.2001, leg. Wolf (cAss); $1 \sigma^{\star}, 14$ exs., Calabria, Catena Costiera, L. Trifoglietti, 1300 m, leg. Angelini \& Sabella (MNB, cAss); 1 ㅇ, Catena Costiera, Fuscaldo (CS), 900 m, 5.VII.1988, leg. Angelini (cAss); 1 ㅇ [teneral], Monti Orsomarso, Grisolia, Pantanelli (CS), 670 m, 4.VIII.1989, leg. Angelini (cAss); 1 운, Le Serre, M. Pecoraro (CZ), 1250 m, 27.VI.1987, leg. Angelini (cAss); $1 \sigma^{\star}, 1$ 우, Aspromonte, Santuario della Madonne di Polsi, 1000 m, 23.VI.1987, leg. Angelini (cAss); $1 \sigma^{*}$, Piano Aspromonte (RC), 1000 m, swamp, 1.V.1993, leg. Angelini \& Sabella (cAss); $10^{*}, 2$ exs., same data, but 12.VIII.1988, leg. Angelini (cAss); 1 o $^{\star}$, 3 exs., Aspromonte, Gambarle, M. Basilico, $1100 \mathrm{~m}$, 1.V.1993, leg. Angelini (cAss); $1 \sigma^{*}$, Aspromonte, Passo di Cancelo, 950 m, 19-30.VI.2001, leg. Wolf (MNB); $10^{\star}$, 1 ․ Fagnano Castello (CS), Lago Due Uomini, 39³3' $\mathrm{N}$, $16^{\circ} 01^{\prime} \mathrm{E}, 1000 \mathrm{~m}, 25 . \mathrm{IV} .2015$, leg. Tagliapietra \& Zanetti (cZan); 8 exs., Aspromonte (FMNH, NHMW); 7 exs., locality not specified (NHMW).

Croatia: $70^{\top} o^{\top}, 8$ 우 우, Biokovo Planina, road to Sveti Jure, 1500 m, 9.VI.2006, leg. Schuh (cAss); 8 exs., Istria, Rijeka region, Primorje Gorski Kotar, Ucka, 1300-1350 m, 5.VI.2014, leg. Wolf (MNB, cAss); $2 \sigma^{\star} \sigma^{\star}, 2$ exs., Mount Biokovo, leg. Besuchet (MHNG, cAss); $10^{\star}, 1$ ㅇ, Velebit, "Costaria S", leg. Leonhard (FMNH).

Montenegro: $2 \sigma^{\star} \sigma^{*}, 6$ exs., Radoštak, leg. Paganetti (NHMW, cAss); 1 우, locality not specified, leg. Reitter (NHMW); 2 우 우, Kameno, leg. Paganetti (FMNH); $1 \sigma^{\star}, 5$ exs., Krivošije, leg. Paganetti, Bierig, Matcha (FMNH). 
Bosnia-Herzegovina: $1 \sigma^{\star}$, Mostar, Nevesinje, 14.VIII. 1977, leg. Lohse (cAss); 1 ơ, Gromiljak, 400 m, 5.V.1990, leg. Wunderle (cWun); 2 exs., Bjelasnica Planina, leg. Leonhard (NHMW, cAss); 1 ex., Bjelasnica Planina, 1901 (FMNH); 1 ex., Majevica Planina (NHMW); 4 우 우, Sarajevo, V.1907 (NHMW); $10^{\star}, 1$ 우, Jablanica, leg. Formanek, Paganetti (FMNH); $1 \sigma^{*}$, Trebevic, leg. Bierig (FMNH).

Greece: $13 \sigma^{\star} o^{\star}, 16$ ㅇ ․, Makedhonía, NW Kavála, Pangéo, near ski resort, beech forest, 1700 m, 24.V.1999, leg. Assing (cAss); $7 o^{\star} o^{\star}, 8$ 우 우, same data, but $1650 \mathrm{~m}$ (cAss); $3 o^{-1} o^{*}, 1$ ㅇ, same data, but $1650 \mathrm{~m}, 28 . \mathrm{V} .1999$ (cAss); $2 o^{\top} o^{\top}, 1$ 우 , same data, but $1200 \mathrm{~m}, 28$. V.1999 (cAss); $1 \sigma^{\star}$, same data, but road to ski resort, $1350 \mathrm{~m}$, 24.V.1999 (cAss); 4 o o, 5 우, Makedhonía, Dráma, Falakró, road to ski resort, 1500 m, pine forest, 26.V.1999, leg. Assing (cAss); $2 \sigma^{\star} \sigma^{\star}$, Makedhonía, Athos, leg. Schatzmayr (FMNH); $2 \sigma^{\star} \sigma^{\star}, 4$ 우 우, same data, but $1000 \mathrm{~m}$, beech forest (cAss); 3 exs., Makedhonía, Drama region, West Rodopi, N Skaloti, Elatia Old Forest Village env., 1600 m, 13-14.VI.2002, leg. Wolf (MNB, cAss); $1 \sigma^{\star}$, E Thessaloniki, Chortiatis, 28.VI.1960, leg. Kühnelt (NHMW); 7 exs., Chalkidiki, $12 \mathrm{~km}$ NW Megali Panagia, $40^{\circ} 28^{\prime} \mathrm{N}$, $23^{\circ} 34^{\prime} \mathrm{E}, 1000$ m, 21.V.2005, leg. Bayer (MNB, cAss); 1 우, $19 \mathrm{~km}$ NW Drama, Falakro, road to ski resort, $41^{\circ} 18^{\prime} \mathrm{N}$, $24^{\circ} 00^{\prime} \mathrm{E}, 1200 \mathrm{~m}, 14 . \mathrm{V} .2005$, leg. Bahr et al. (MNB); $20^{\star} 0^{\star}, 5$ 우 우, Evvoia, Oros Dirfys, N Kato Steni, $38^{\circ} 37^{\prime} \mathrm{N}$, $23^{\circ} 52^{\prime} \mathrm{E}, 1120 \mathrm{~m}$, fir forest, 8.IV.2001, leg. Assing (cAss); 4 exs., Evvoia, Limni, Drimona, Oros Xiros, above waterfall, 22-26.III.2016, leg. Esser \& Niefeld (MNB, cAss); $6{o^{\star}}^{\star} o^{\star}, 8$ 우 우, 4 exs., Fokis, 25 km SSW Lamia, Kaloskopi, $38^{\circ} 42^{\prime} \mathrm{N}, 22^{\circ} 18^{\prime} \mathrm{E}, 1200 \mathrm{~m}$, fir forest, 6.IV.2001, leg. Assing $\&$ Wunderle (cAss, cWun); $10^{*}$, Fokis, Oros Lidorikiou, 1170 m, 11.VI.1991, leg. Giachino \& Vailati (cAss); $10^{*}$, Fokis, Oros Giona, $38^{\circ} 40^{\prime} \mathrm{N}, 2^{\circ}{ }^{\circ} 19^{\prime} \mathrm{E}, 1650 \mathrm{~m}$, 17.IV.2000, leg. Wunderle (cWun); $10^{\star}, 1$ ㅇ, Fokis, Oros Vardousia, W Diakofto, $38^{\circ} 42^{\prime} \mathrm{N}, 22^{\circ} 10^{\prime} \mathrm{E}, 1540 \mathrm{~m}$, fir forest, 18.IV.2000, leg. Wunderle (cWun); $10^{*}, 2$ 우 오․ Fthiotis, SSE Lamia, Oros Kallidromo, $38^{\circ} 44^{\prime} \mathrm{N}, 22^{\circ} 32^{\prime} \mathrm{E}, 1250 \mathrm{~m}$, fir forest, 7.IV.2001, leg. Assing (cAss); $100^{\top} 0^{*}, 6$ 우 우, 5 exs., SSE Lamia, Oros Kallidromo, $38^{\circ} 45^{\prime} \mathrm{N}, 22^{\circ} 32^{\prime} \mathrm{E}$, $1200 \mathrm{~m}$, fir forest, 7.IV.2001, leg. Assing \& Wunderle (cAss, cWun).

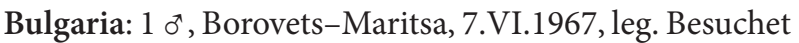
(MHNG); 1 ㅇ, Skakawitza, leg. Blagoeff (MHNG).

Turkey: Kirklareli: $1 o^{\star}, 1$ ex., Yeniceköy-Demirköy, 31.VII.1969, leg. Besuchet (MHNG, cAss). Canakkale: $20^{\top} o^{\star}$, Kaz Dağ 1 , Ayazma, $39^{\circ} 45^{\prime} \mathrm{N}, 26^{\circ} 54^{\prime} \mathrm{E}, 440 \mathrm{~m}$, 11.IV.2010, leg. Brachat \& Meybohm (cAss); 2 우 우, Kurucam Tepe, $39^{\circ} 43^{\prime} \mathrm{N}, 27^{\circ} 11^{\prime} \mathrm{E}, 430 \mathrm{~m}, 14 . I V .2010$, leg. Brachat \& Meybohm (cAss). Balıkesir: 1 우, Kaz Dağı, Ayazma, $39^{\circ} 45^{\prime} \mathrm{N}, 26^{\circ} 51^{\prime} \mathrm{E}, 500 \mathrm{~m}, 11 . I V .2010$, leg. Brachat \& Meybohm (cAss); 1 ㅇ, Kurucam Tepe, $39^{\circ} 41^{\prime} \mathrm{N}, 27^{\circ} 10^{\prime} \mathrm{E}, 740 \mathrm{~m}, 13 . \mathrm{IV} .2010$, leg. Brachat \& Meybohm (cAss). Bursa: 2 우 오, Karaorman, 39 $55^{\circ} \mathrm{N}$, $28^{\circ} 28^{\prime} \mathrm{E}, 440 \mathrm{~m}, 15 . \mathrm{IV} .2010$, leg. Brachat \& Meybohm (cAss); $1 \mathrm{o}^{\top}$, Devecikonağ $1,39^{\circ} 50^{\prime} \mathrm{N}, 28^{\circ} 26^{\prime} \mathrm{E}, 640 \mathrm{~m}$, 16.IV.2010, leg. Brachat \& Meybohm (cAss); 1 ㅇ, Uludağ, $40^{\circ} 08^{\prime \prime} \mathrm{N}, 2^{\circ} 02^{\prime} \mathrm{E}, 1030 \mathrm{~m}, 17 . I V .2010$, leg. Brachat \& Meybohm (cAss); 11 exs., Uludağ, 1300 m, 12.V.1976, leg. Besuchet \& Löbl (MHNG, cAss); 5 exs., Uludağ, $1500 \mathrm{~m}, 13 . \mathrm{V} .1976$, leg. Besuchet \& Löbl (MHNG); 2 우 오, Soğukpınar, $40^{\circ} 05^{\prime} \mathrm{N}, 2^{\circ} 05^{\prime} \mathrm{E}, 1220 \mathrm{~m}, 18 . \mathrm{IV} .2010$, leg. Brachat \& Meybohm (cAss); $20^{\top} o^{\top}, 1$ ㅇ, Kocayayla, $39^{\circ} 56^{\prime} \mathrm{N}, 29^{\circ} 16^{\prime} \mathrm{E}, 1200 \mathrm{~m}, 18 . \mathrm{IV} .2010$, leg. Brachat

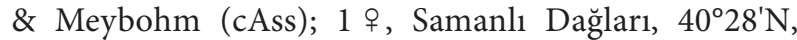
$29^{\circ} 49^{\prime} \mathrm{E}, 780 \mathrm{~m}$, 19.IV.2010, leg. Brachat \& Meybohm

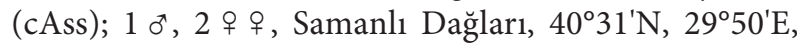
$720 \mathrm{~m}, 19 . \mathrm{IV} .2010$, leg. Brachat \& Meybohm (cAss); $10^{\star}, 1$ 우, Elmall, $40^{\circ} 31^{\prime} \mathrm{N}, 29^{\circ} 54^{\prime} \mathrm{E}, 1000 \mathrm{~m}, 20 . I \mathrm{~V} .2010$, leg. Brachat \& Meybohm (cAss); 4 exs., S Bursa, 500 m, 12.V.1976, leg. Besuchet \& Löbl (MHNG); 1 ex., S Bursa, 22.VII.1969, leg. Besuchet (MHNG). Kocaeli: 1 ㅇ, N Hereke, $40^{\circ} 49^{\prime} \mathrm{N}, 29^{\circ} 41^{\prime} \mathrm{E}, 460 \mathrm{~m}, 6 . \mathrm{V} .2012$, leg. Brachat \& Meybohm (cAss). Sakarya: $10^{\star}, 2$ 우 잉 $17 \mathrm{~km}$ $\mathrm{N}$ Hendek, $40^{\circ} 53^{\prime} \mathrm{N}, 30^{\circ} 46^{\prime} \mathrm{N}, 650 \mathrm{~m}, 3 . \mathrm{V} .2012$, leg. Brachat \& Meybohm (cAss). Bolu: 1 , Yedigöller Milli Park, $40^{\circ} 53^{\prime} \mathrm{N}, 31^{\circ} 41^{\prime} \mathrm{N}, 1720 \mathrm{~m}, 28 . I V .2012$, leg. Brachat \& Meybohm (cAss); $10^{\star}, 2$ 우 우, $25 \mathrm{~km} \mathrm{~S}$ Bolu, $40^{\circ} 38^{\prime} \mathrm{N}$, $31^{\circ} 37^{\prime} \mathrm{N}, 1580 \mathrm{~m}, 30 . I V .2012$, leg. Brachat \& Meybohm (cAss); 2 우 우, Kartalkaya, $40^{\circ} 36^{\prime} \mathrm{N}, 31^{\circ} 48^{\prime} \mathrm{E}, 1820 \mathrm{~m}$, 25.IV.2014, leg. Brachat \& Meybohm (cAss); $4 o^{\top} o^{\top}$, 4 우 우, Kartalkaya, $40^{\circ} 37^{\prime} \mathrm{N}, 31^{\circ} 49^{\prime} \mathrm{E}, 1720$ m, 25.IV.2014, leg. Brachat \& Meybohm (cAss); 6 exs., 9 km N Mengen, $750 \mathrm{~m}, 23 . \mathrm{V} .1976$, leg. Besuchet \& Löbl (MHNG); 13 exs., Abant, 1500-1600 m, 22.V.1976, leg. Besuchet \& Löbl (MHNG); 2 exs, Abant, 1400 m, 25.V.1967, leg. Besuchet (MHNG); 1 ex., Bolu env., Ömerler, 800 m, 21.V.1976, leg. Besuchet \& Löbl (MHNG). Zonguldak: $2 o^{\top} o^{\star}, 1$ 우, ca. $15 \mathrm{~km}$ W Devrek, $41^{\circ} 14^{\prime} \mathrm{N}, 31^{\circ} 52^{\prime} \mathrm{E}$, $700 \mathrm{~m}$, beech forest with Rhododendron undergrowth, litter sifted, 26.III.2010, leg. Assing (cAss); 18 exs., S Zonguldak, 500 m, 23.V.1976, leg. Besuchet \& Löbl (MHNG); 2 exs., 17 km N Safranbolu, 1000 m, 16.V.1976, leg. Besuchet \& Löbl (MHNG). Karabük: 1 ㅇ , pass W Eskipazar, $40^{\circ} 58^{\prime} \mathrm{N}, 33^{\circ} 23^{\prime} \mathrm{E}, 1400 \mathrm{~m}$, beech forest, litter sifted, 26.III.2010, leg. Assing (cAss). Kastamonu: 1 ㅇ, Ilgaz Dağ 1 , Ballık, $41^{\circ} 12^{\prime} \mathrm{N}, 33^{\circ} 48^{\prime} \mathrm{E}, 1040 \mathrm{~m}$, 26.IV.2014, leg. Brachat \& Meybohm (cAss); 1 ㅇ, Ilgaz Dağ 1 , Tüfekçi, $41^{\circ} 06^{\prime} \mathrm{N}, 33^{\circ} 44^{\prime} \mathrm{E}, 1380 \mathrm{~m}, 27 . I V .2014$, leg. Brachat \& Meybohm (cAss); $10^{\star}, \mathrm{S}$ Küre, $41^{\circ} 45^{\prime} \mathrm{N}$, $33^{\circ} 42^{\prime} \mathrm{E}, 1060 \mathrm{~m}, 27 . I V .2014$, leg. Brachat \& Meybohm (cAss); $3 o^{\star} o^{\star}, 4$ 우 우, N Küre, $41^{\circ} 53^{\prime} \mathrm{N}, 33^{\circ} 42^{\prime} \mathrm{E}, 880 \mathrm{~m}$, 27-28.IV.2014, leg. Brachat \& Meybohm (cAss); 4 exs., Inebolu-Küre, 700 m, 18.V.1976, leg. Besuchet \& Löbl (MHNG); 28 exs., 5 km S Küre, 1200 m, 18.V.1976, leg. Besuchet \& Löbl (MHNG, cAss); 5 exs., 5 km N Küre, 600 m, 18.V.1976, leg. Besuchet \& Löbl (MHNG); 4 exs., S Inebolu, $600 \mathrm{~m}, 18 . V .1976$, leg. Besuchet \& Löbl (MHNG); 3 exs., pass 15 km N Tosya, Ilgaz Dağı, 16001700 m, 19.V.1976, leg. Besuchet \& Löbl (MHNG); 1 ex., Ilgaz Dağ1, Diphan, 1300 m, 17.V.1976, leg. Besuchet \& Löbl (MHNG); 7 exs., 32 km N Tosya, Karadere, 1400 m, 19.V.1976, leg. Besuchet \& Löbl (MHNG); 5 exs., 13 km E Ağli, 1200 m, 18.V.1976, eg. Besuchet \& Löbl (MHNG). Sinop: 1 ․ SSW Sinop, $41^{\circ} 43^{\prime} \mathrm{N}$, 
$34^{\circ} 55^{\prime} \mathrm{E}, 660 \mathrm{~m}$, oak forest, sifted, 29.III.2009, leg. Assing (cAss); $10^{\star}, 1$ ㅇ, ca. $30 \mathrm{~km} \mathrm{~S} \mathrm{Ayancık,} \mathrm{Çangal} \mathrm{Dağ} 1$, $41^{\circ} 49^{\prime} \mathrm{N}, 34^{\circ} 39^{\prime} \mathrm{E}, 920 \mathrm{~m}$, beech forest, sifted, 1.IV.2009, leg. Assing (cAss); 8 exs., Çangal Dağı, V.1957, leg. Schubert (NHMW); 6 exs., same data, but VI.1959; 11 exs., same data, but VI.1960 (NHMW); 2 exs., $29 \mathrm{~km}$ N Boyabat, Bürnük, 1250 m, 20.V.1976, leg. Besuchet \& Löbl (MHNG); 10 exs., 23 km N Boyabat, S Bektaş, $1100 \mathrm{~m}, 20 . V .1976$, leg. Besuchet \& Löbl (MHNG). Samsun: 1 ㅇ, $21 \mathrm{~km}$ NNE Havza, $41^{\circ} 09^{\prime} \mathrm{N}, 35^{\circ} 45^{\prime} \mathrm{E}$, $950 \mathrm{~m}$, Quercus-Carpinus forest, litter and moss sifted, 19.VII.2008, leg. Assing (cAss); 1 ㅇ, $25 \mathrm{~km} \mathrm{~S} \mathrm{Samsun,}$ $\mathrm{NE}$ Asarc1k, $41^{\circ} 05^{\prime} \mathrm{N}, 36^{\circ} 16^{\prime} \mathrm{E}, 880 \mathrm{~m}$, Fagus-QuercusCarpinus forest, litter in dry stream sifted, 20.VII.2008,

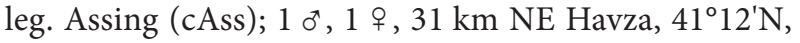
$35^{\circ} 52^{\prime} \mathrm{E}, 670 \mathrm{~m}$, beech forest, litter sifted, 19.VII.2008, leg. Assing (cAss). Ordu: 1 ex., $34 \mathrm{~km}$ SSE Gölköy, W Mesudiye, $40^{\circ} 26^{\prime} \mathrm{N}, 37^{\circ} 47^{\prime} \mathrm{E}, 1520 \mathrm{~m}, 14$. VII.2008, leg. Schülke (MNB). Gümüşhane: 1 ㅇ, 1 ex., ca. $25 \mathrm{~km}$ SW Gümüşhane, Tersundağ $\mathrm{Geçidi,} 40^{\circ} 18^{\prime} \mathrm{N}, 39^{\circ} 18^{\prime} \mathrm{E}$, $2070 \mathrm{~m}, \mathrm{~N}$-slope, spruce forest, litter and dead wood sifted, 24.VII.2006, leg. Assing (cAss, MNB); $10^{\text {}}$, ca. $50 \mathrm{~km}$ SW Trabzon, NE Kürtün, $40^{\circ} 42^{\prime} \mathrm{N}, 39^{\circ} 15^{\prime} \mathrm{E}$, 1750 m, 27.VII.2006, leg. Schülke (MNB).
Diagnosis: Winged species of mostly reddish coloration. $0^{7}$ : median lobe of aedeagus (Figs 23-26) of distinctive shape, relatively large, approximately $0.45 \mathrm{~mm}$ long; ventral process strongly curved in lateral view and apically concave in ventral view; crista apicalis very small; crista basalis very narrow; apical lobe of paramere (Fig. 27) relatively short, barely half as long as basal portion; basal portion of paramere (Fig. 27) with distinctive dark structure and relatively short apical lobe.

क: spermatheca (Fig. 28) of variable shape, relatively small, with bell-shaped apical cuticular invagination.

Distribution and natural history: The revised distribution of $O$. nova is remarkably discontinuous (Maps 1,5). In Italy, it is confined to the extreme south (Calabria). This population is separated from the geographically closest populations in the Balkans by the Adriatic and Ionian Seas or by approximately $1000 \mathrm{~km}$ on land (North Croatia). In the Balkans, it is distributed in the northwest (Croatia, Bosnia-Herzegovina, Montenegro) and in the southeast (Northeast Greece, Bulgaria); moreover, there is an enclave in the south of mainland Greece. These regions are separated by the distributional range of O. kuehnelti. From the southeastern Balkans, the

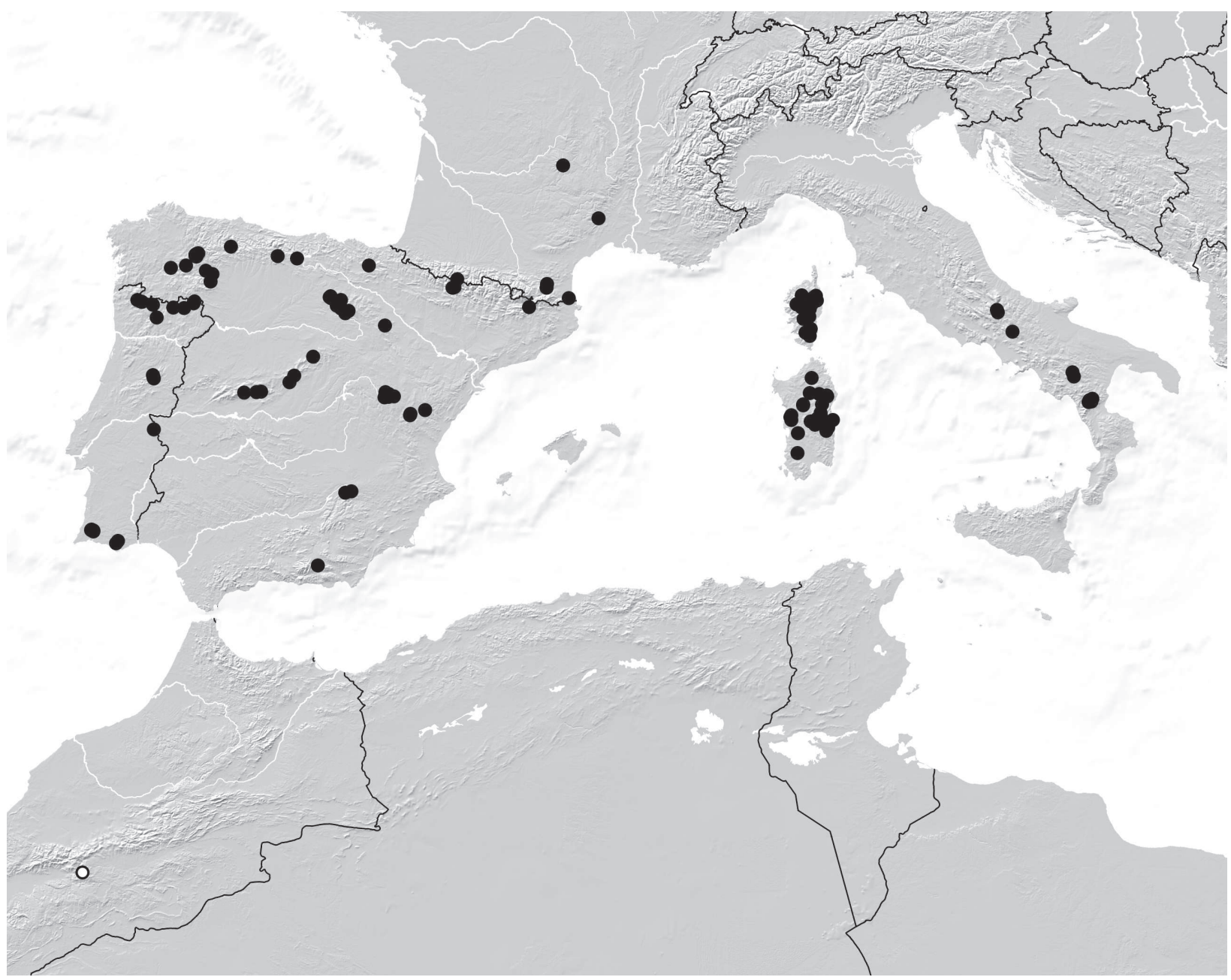

Map 4: Distribution of Oxypoda fusina in the West Mediterranean, based on revised records (white circle: doubtful female-based record from Morocco). 


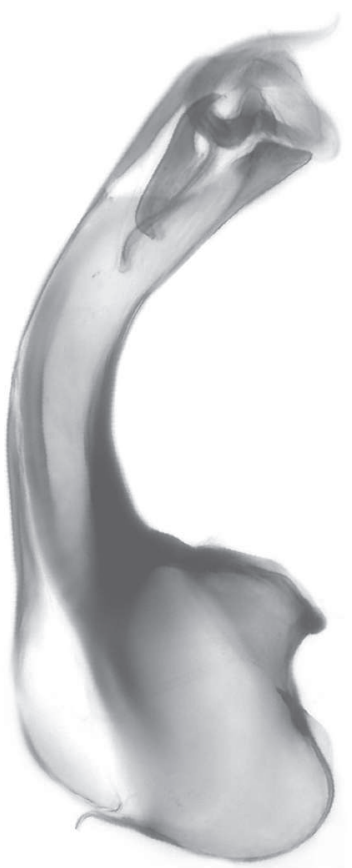

23

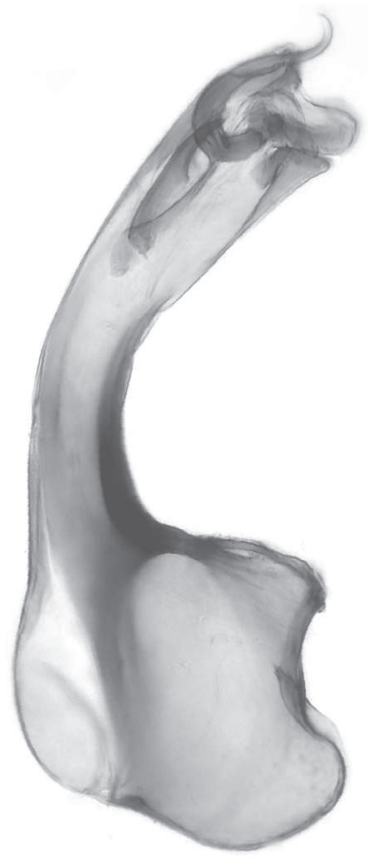

24

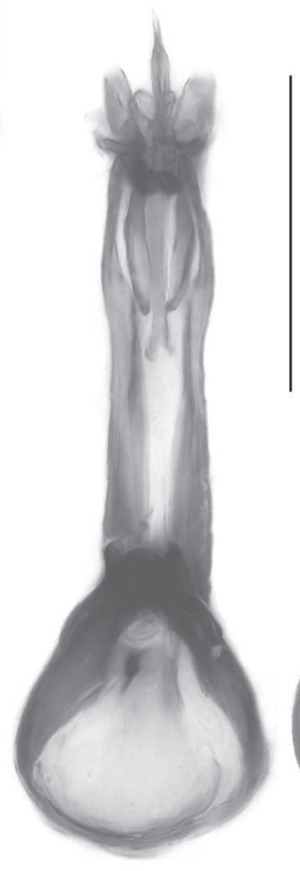

25

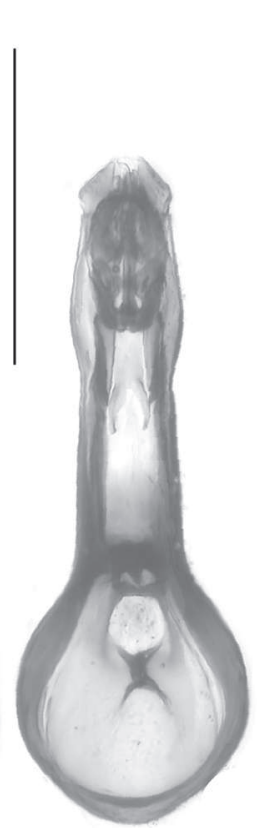

33

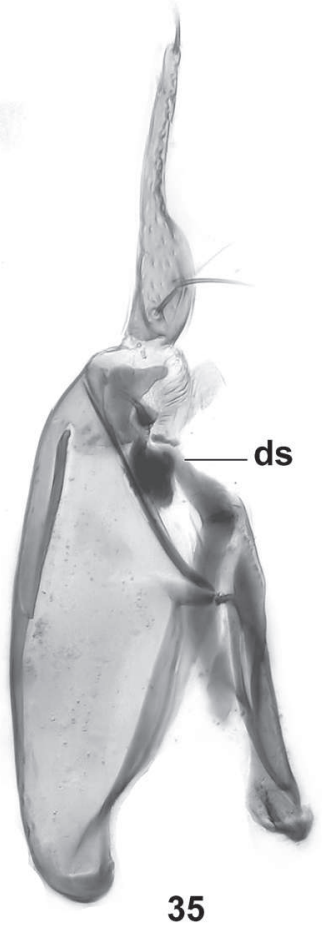

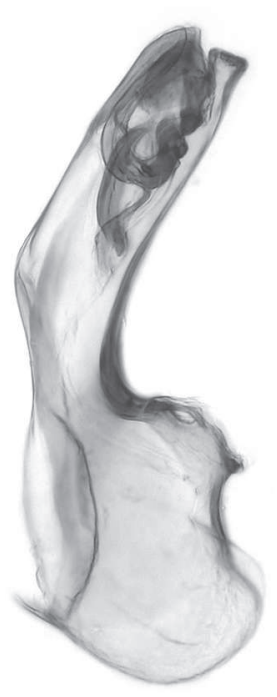

29

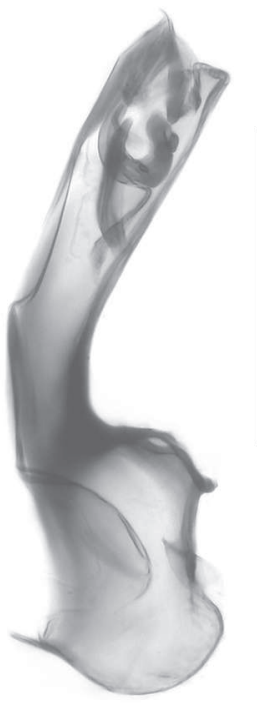

30

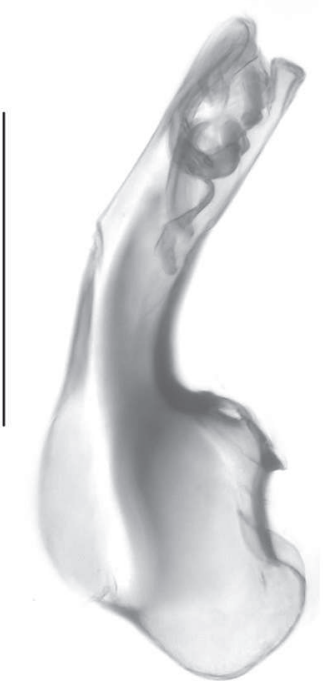

31

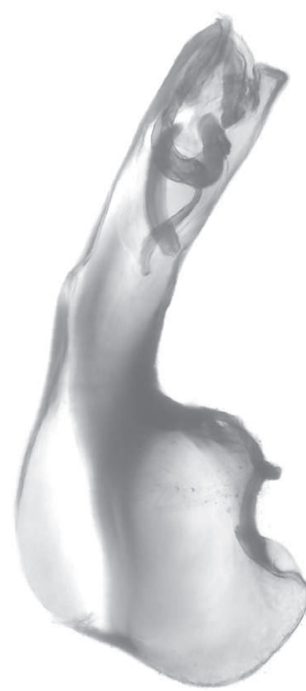

32

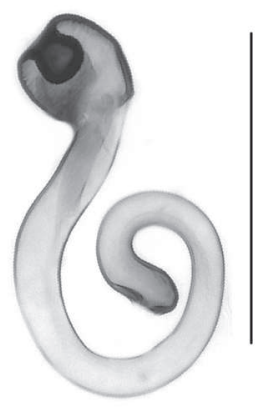

28

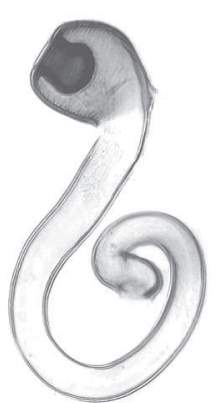

36

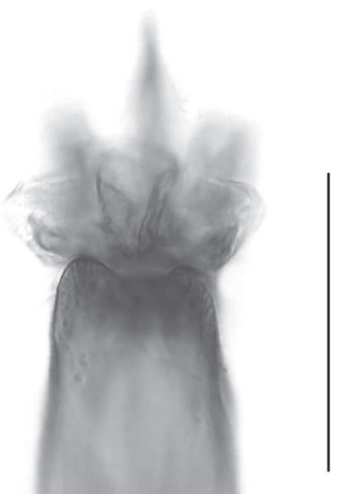

26

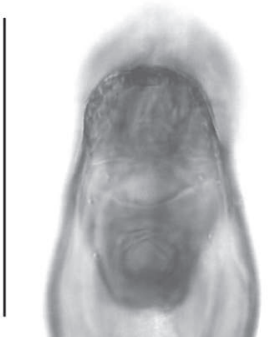

34

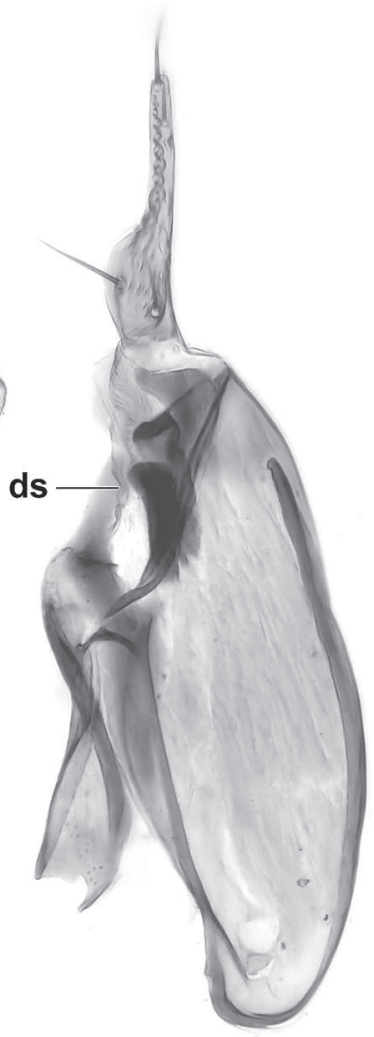

27

Figs 23-36: Oxypoda nova from South Italy $(23,25-27)$ and Croatia $(24,28)$, and O. kuehnelti $(29-36)$ : median lobe of aedeagus in lateral and in ventral view (23-25, 29-33); apex of median lobe of aedeagus in ventral view $(26,34)$; paramere $(27,35$; "ds" = dark structure); spermatheca $(28,36)$. Scale bars: 23-25, 27-33, 35-36: $0.2 \mathrm{~mm} ; 26,34: 0.1 \mathrm{~mm}$. 
range continues along North Anatolia eastwards to Gümüşhane and overlaps with that of O. caucasica. The only plausible explanation of this remarkable distribution pattern is interspecific competition with other Baeoglena species (O. fusina, O. kuehnelti, O. rectacia, possibly also O. caucasica).

The examined material was mostly sifted from leaf litter and roots in various forest (deciduous, coniferous, mixed) and bush habitats, but was also found in swamps and on stream banks. The altitudes range from 400 to $2070 \mathrm{~m}$. The specimens were found in March (4 records), April (28), May (35), June (12), July (9), and August (3). The respective number of records most likely reflects the collecting activity more than the actual phenology of the species.

\section{Oxypoda (Baeoglena) kuehnelti ScheERPELTz, 1963 (Figs 29-39, Maps 1, 5)}

Oxypoda (Baeoglena) Kühnelti Scheerpeltz, 1963: 449 ff.

Type material examined:Lectotype $\sigma^{\star}$, present designation: "o* / Prioni, 1600 m, Olymp, 3.V.60 / Griechenland, W. Kühnelt / Oxypoda (Baeoglena) Kühnelti nov. spec. / ex coll. Scheerpeltz / Typus Oxypoda (Baeoglena) Kühnelti O. Scheerpeltz / Oxypoda praecox Erichson, M. Tronquet det. 2000 / Lectotypus o Oxypoda kuehnelti Scheerpeltz, desig. V. Assing 2018 / Oxypoda kuehnelti Scheerpeltz, det. V. Assing 2018" (NHMW). Paralectotype of [dissected prior to present study; specimen completely bleached, pale-yellow]: same data as lectotype (NHMW).

Comment: The original description is based on " $10^{\star}$, 1 , Typen" (i.e., syntypes) from "Prioni ... im OlympMassiv" (ScheERPeltz 1963). The male is designated as the lectotype.
Material examined: Serbia: $10^{\star}, 3$ ㅇ + , Niš region, Suva

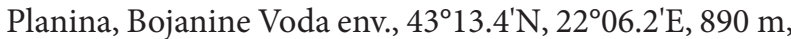
sifted, 2.VII.2006, leg. Hlaváč (cAss); 3 우, same data, but 1100-1200 m, 3.VII.2006 (cAss); 5 exs., Niš region, Suva Planina, Bojanine Voda env., $43^{\circ} 13.4^{\prime} \mathrm{N}, 22^{\circ} 06.2^{\prime} \mathrm{E}$, $850 \mathrm{~m}$, forest litter sifted, 2.VII.2006, leg. Cooter (cAss); 1 ex., Suva Planina, Bojanine Voda env.,1100 m, 3.VII.2006, leg. Cooter (cAss); 1 o , 1 오, Suva Planina, Smrdan, 1650 m, 17.V.2007, leg. Stévanović (cAss); $2 \sigma^{*} o^{*}$, Svrljiške Planina, Lozan, Bare, 10.VII.2009, leg. Stévanović (cAss); 1 ex., $2 \sigma^{\star} o^{\star}$, Svrljiške Planina, Ribarska korita, 3.VII.2006, leg. Cooter (cAss); 2 o $^{\star} o^{\star}, 5$ 우 우, Surijiške Planina, Prekonoge env., 4.VII.2006, leg. Hlaváč (cAss); $10^{\star}, 4$ 우, Niš region, Babička Gora, Donji

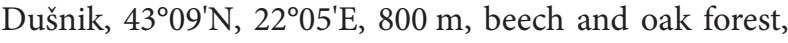
5.VII.2006, leg. Hlaváč (cAss); 5 exs., Niš region, Babička Gora, Siroka Padina, 750 m, beech litter, 5.VII.2006, leg. Cooter (cAss); 1 đ , Babička Gora, Zelenika, 20.VII.2006, leg. Stevanovič (cFel); $1 \sigma^{\star}, 1$ ㅇ, Kapaonik mts., Jaram, 1550 m, 6.VI.2009, leg. Stévanović (cAss); 1 ơ, Javorak Planina, Grza, 720 m, 8.IV.2007, leg. Stévanović (cAss); 1 o , 2 exs., Rtanj Planina, 600 m, 27.V.1984, leg. Besuchet (MHNG, cAss).

Albania: 3 exs., Tiranë, 10 km ENE Tiranë, Mali Dajti, $41^{\circ} 22^{\prime} \mathrm{N}, 19^{\circ} 55^{\prime} \mathrm{E}, 1160 \mathrm{~m}$, mixed beech forest, litter sifted, 21.V.2010, leg. Assing (cAss); 3 exs., Elbasan, $13 \mathrm{~km}$ SE Elbasan, Mali i Shpatit, $41^{\circ} 03^{\prime} \mathrm{N}, 20^{\circ} 13^{\prime} \mathrm{E}, 1500 \mathrm{~m}$, beech forest margin, sifted, 22.V.2010, leg. Assing (cAss); 6 exs., Librazhd, $25 \mathrm{~km}$ ESE Elbasan, Mali i Polisit, $41^{\circ} 04^{\prime} \mathrm{N}, 20^{\circ} 22^{\prime} \mathrm{E}, 1700 \mathrm{~m}$, old beech forest with snow, litter sifted, 23.V.2010, leg. Assing (cAss); 12 exs., Korçë, $37 \mathrm{~km}$ W Korçë, Mali i Ostrovikës, $40^{\circ} 40^{\prime} \mathrm{N}, 20^{\circ} 21^{\prime} \mathrm{E}$, $1340 \mathrm{~m}$, beech forest margin, beech litter and fern roots sifted, 26.V.2010, leg. Assing (cAss); 19 exs., Korçë, 10 km S Korçë, Mali i Gramozit, $40^{\circ} 32^{\prime} \mathrm{N}, 20^{\circ} 48^{\prime} \mathrm{E}, 1570 \mathrm{~m}$, beech forest with poplar and juniper, sifted, 27.V.2010, leg. Assing (cAss, MNB); 7 exs., Korçë, 10 km S Korçë, Mali i Gramozit, $40^{\circ} 31^{\prime} \mathrm{N}, 20^{\circ} 48^{\prime} \mathrm{E}, 1550 \mathrm{~m}$, moist beech

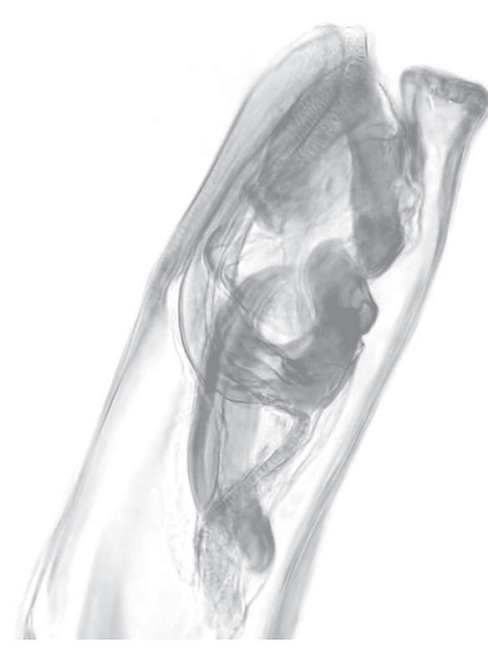

37

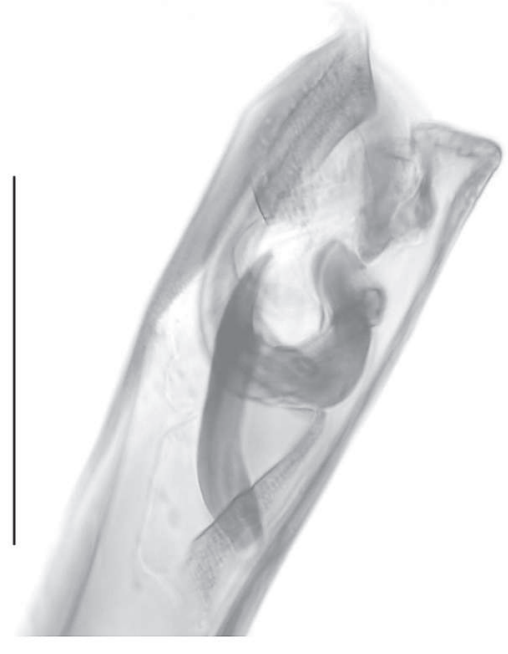

38

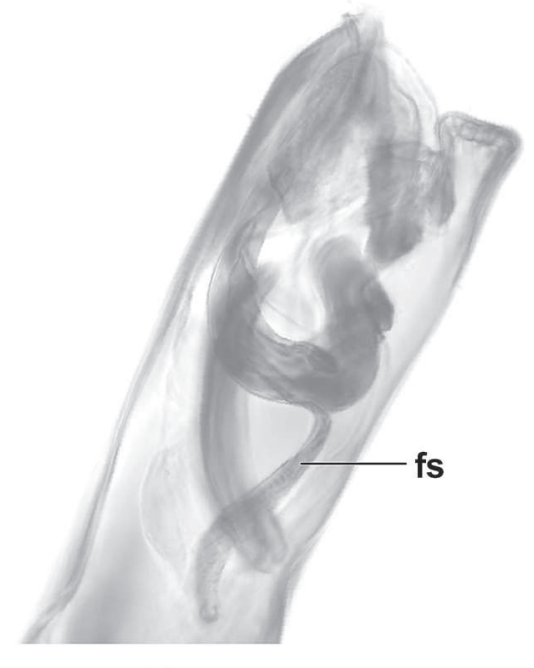

39

Figs 37-39: Oxypoda kuehnelti: apex of median lobe of aedeagus in lateral view; "fs" = apico-ventral filiform structure. Scale bar: $0.1 \mathrm{~mm}$. 
forest margin, beech, fern, etc. sifted, partly near temporary stream, 27.V.2010, leg. Assing (cAss); 10 exs., Mount Tomorr [ $\left.40^{\circ} 42^{\prime} \mathrm{N}, 20^{\circ} 08^{\prime} \mathrm{E}\right]$, Kulmak pass, V.1931, leg. Winkler (NHMW, cAss).

Macedonia: $6 \sigma^{\top} \sigma^{\star}$, Bistra Mts., Mavrovo env., $1540 \mathrm{~m}$, sifted, 16.VI.2011, leg. Hlaváč (cAss); 1 o , Šar planina, Popova Šapka, 16.VII.1935, leg. Fodor (HNHM); 6 exs., Šar planina, Popova Šapka, 5-11.VI.1955, leg. Schubert (NHMW); $10^{*}, 1$ ㅇ, E Ohrid lake, Galičica, 18002000 m, beech litter sifted, 18-21.VII.1964, leg. Knappe (NHMW).

Greece: 17 exs., N-Pindos, ca. 40 km NNE Konitsa, Oros Gramos range, S Oros Arénes, SE Aetomilitsa, $40^{\circ} 18^{\prime} \mathrm{N}$, $20^{\circ} 54^{\prime} \mathrm{E}, 1720 \mathrm{~m}$, beech forest, 25.V.2005, leg. Assing (cAss); 55 exs., N-Pindos, ca. 40 km NNE Konitsa, Oros Gramos range, S Oros Arénes, SE Aetomilitsa, $40^{\circ} 18^{\prime} \mathrm{N}$, $20^{\circ} 53^{\prime} \mathrm{E}, 1650 \mathrm{~m}$, beech forest, sifted, 25.V.2005, leg. Assing (cAss); 1 ex., same data, but grass near forest margin sifted (cAss); 2 exs., Ioannina, ca. $15 \mathrm{~km}$ SE Konitsa, Oros Timfi, W Vrisochori, 39²59'N, $20^{\circ} 53^{\prime} \mathrm{E}, 1280 \mathrm{~m}$, beech forest, 26.V.2005, leg. Assing (cAss); 1 ㅇ, Ioánnina, Oros Smólikas, above Palioseli, $40^{\circ} 04^{\prime} \mathrm{N}, 20^{\circ} 53^{\prime} \mathrm{E}, 1630 \mathrm{~m}$, subterranean pitfall trap, 17.VI.2007-16.VI.2008, leg. Giachino \& Vailati (cAss); $1 \mathrm{o}^{\star}$, Ioánnina, Oros Timfi, road to Vovoússa, $1480 \mathrm{~m}$, subterranean pitfall trap, 27.VI.2004-16.VI.2005, leg. Giachino \& Vailati (cAss); 2 exs., Ioánnina, Notia Pindos, Anilio, road to ski resort, $39^{\circ} 45^{\prime} \mathrm{N}, 21^{\circ} 12^{\prime} \mathrm{E}$, 16.IV.2018, leg. Schülke (MNB, cAss); $10^{\star}$, Ioánnina, Notia Pindos, Metsovo, 1400 m, 24.VI.1997, leg. Bayer

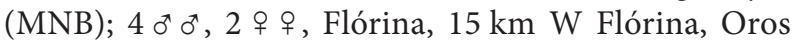
Varnous, $40^{\circ} 48^{\prime} \mathrm{N}, 21^{\circ} 15^{\prime} \mathrm{E}, 1600 \mathrm{~m}$, 23.IV.2000, leg. Assing \& Wunderle (cAss, cWun); $30^{\top} o^{\star}, 2$ 우 우, 6 exs., Flórina, $15 \mathrm{~km}$ S Flórina, Oros Vitsi, $40^{\circ} 39^{\prime} \mathrm{N}, 21^{\circ} 23^{\prime} \mathrm{E}$, 1850-1880 m, 25.IV.2000, leg. Assing \& Wunderle (cAss, cWun); $10^{\star}$, Flórina, Oros Vitsi, above Drosopigi, $40^{\circ} 39^{\prime} \mathrm{N}, 21^{\circ} 24^{\prime} \mathrm{E}, 1340 \mathrm{~m}$, subterranean pitfall trap, 19.VI.2007-17.VI.2008, leg. Giachino \& Vailati (cAss); $2 o^{\star} o^{\star}, 6$ exs., Flórina, 40 km NE Flórina, Oros Voras, $40^{\circ} 53^{\prime} \mathrm{N}, \quad 21^{\circ} 48^{\prime} \mathrm{E}, 1650 \mathrm{~m}, 22 . I V .2000$, leg. Assing \& Wunderle (cAss, cWun); $20^{\star} \sigma^{*}, 2$ 우 우, Makedhonía, Imatia, Vermio, above Seli, $40^{\circ} 33^{\prime} \mathrm{N}, 22^{\circ} 00^{\prime} \mathrm{E}$, $1500 \mathrm{~m}$, 11.IV.1998, leg. Assing \& Schülke (cAss, MNB); 2 exs., Makedhonía, Imathia, Vermio, NW Kato Vermio, 1300 m, 6.VI.1999, leg. Brachat (MNB, cAss); 8 exs., Sedi near Katovermio, 1300-1500 m, V.1979, leg. Franz (NHMW); 1 ơ, 1 , Makedhonía, Grevena, Smolikas Oros, SW Smixi, 1450 m, 18.VI.2002, leg. Brachat (cAss); $10^{*}, 1$ ㅇ, Makedhonía, Kilkis, Oros Páiko, Livadia, $40^{\circ} 59^{\prime} \mathrm{N}, 22^{\circ} 18^{\prime} \mathrm{E}, 1115 \mathrm{~m}$, subterranean pitfall trap, 14.IX.2015-7.VI.2017, leg. Giachino \& Vailati (cAss); $2 \sigma^{\star} o^{\star}, 6$ exs., Katara pass, $1500 \mathrm{~m}$, 13.V.1997, leg. Wolf (MNB); 2 o $^{\top}$ o $^{\star}, 7$ exs., Makedhonía, Grevena, Smolika range, pass between Samarina and Fourka, 1700 m, 21.VI.2002, leg. Wolf (MNB, cAss); 10 exs., Grevena, Smolika range, above Smixi, 1450 m, 18-20.VI.2002, leg. Wolf (MNB, cAss); 1 ex., Grevena, Smolika range, W Fourka, 1550 m, 21.VI.2002, leg.
Wolf (MNB); 5 o $^{\star}$, 4 우 ㅇ , 17 exs., Makedhonía, Kozani, Pieria Oros, E Katafigi, $40^{\circ} 16^{\prime} \mathrm{N}, 2^{\circ} 09^{\prime} \mathrm{E}, 1450 \mathrm{~m}$, pine forest, 9.IV.1998, leg. Assing, Schülke \& Wunderle (cAss, cWun, MNB); 7 o $^{\star} \sigma^{\star}, 7$ , 2 exs., Pieria Oros, W Skotina, $40^{\circ} 12^{\prime} \mathrm{N}, 22^{\circ} 10^{\prime} \mathrm{E}, 1000 \mathrm{~m}, 9 . I V .1998$, leg. Assing, Schülke \& Wunderle (cAss, cWun, MNB); $2 \sigma^{\star} \sigma^{*}$, Pieria Oros, above Skotina, $650 \mathrm{~m}$, beech forest, 9.IV.1998, leg. Wunderle (cWun); 1 오 Tríkala, road Píli-Stournaráiika-Mesohóra, Vatirreuma, $920 \mathrm{~m}$, subterranean pitfall trap, 24.VI.2004-14.VI.2005, leg. Giachino \& Vailati (cAss); 4 exs., Trikala, Notia Pindos, W Stournaraika, $39^{\circ} 24^{\prime} \mathrm{N}, 21^{\circ} 28^{\prime} \mathrm{E}, 1330 \mathrm{~m}, 19 . \mathrm{IV} .2018$, leg. Schülke (MNB, cAss); $10^{\star}$, Kardítsa, Belomíti, Kremasta valley near Spilia Ghaki, $39^{\circ} 14^{\prime} \mathrm{N}, 21^{\circ} 43^{\prime} \mathrm{E}$, $1060 \mathrm{~m}$, subterranean pitfall trap, 12.VI.2008-11. VI.2010, leg. Giachino \& Vailati (cAss); $10^{*}$, Kastoriá, Oros Vório, Kotíli, 40²0'N, $21^{\circ} 00^{\prime} \mathrm{E}, 1465 \mathrm{~m}$, subterranean pitfall trap, 17.VI.2008-21.VI.2010, leg. Giachino \& Vailati (cAss); $10^{\star}, 4$ 우, Kastoriá, Oros Vório, Kotíli, $40^{\circ} 21^{\prime} \mathrm{N}, 21^{\circ} 02^{\prime} \mathrm{E}, 1325 \mathrm{~m}$, subterranean pitfall trap, 17.VI.2007-16.VI.2008, leg. Giachino \& Vailati (cAss); $10^{\star}, 1$ q , Kastoriá, Oros Vório, above Kotili, $40^{\circ} 20^{\prime} \mathrm{N}, 21^{\circ} 01^{\prime} \mathrm{E}, 1440 \mathrm{~m}$, subterranean pitfall trap, VI.2017-VI.2018, leg. Giachino \& Vailati (cAss); 1 ơ, Kastoriá, 7 km E Eptachori, 1240 m, 22.V.1999, leg. Brachat (MNB); $4 o^{\star} o^{\star}, 2$ 우 오, Piera, Pieria Oros, W Ritini, 600 m, beech forest, 18.IV.2006, leg. Vít (cAss); $1 \sigma^{\star}$, Piera, Pieria Oros, W Ritini, 600 m, hollow beech trunk, 18.IV.2006, leg. Vít (cAss); 1 †, Pieria, Olympos, W Lithohoro, $400 \mathrm{~m}$, moss and fern sifted, 15.IV.2006, leg. Vít (cAss); $10^{\star}, 1$ ㅇ, Pieriá, Oros Olympos, above Litóhoro, $40^{\circ} 06^{\prime} \mathrm{N}, 22^{\circ} 28^{\prime} \mathrm{E}, 1090 \mathrm{~m}$, subterranean pitfall trap, VI.2017-VI.2018, leg. Giachino \& Vailati; $3 \sigma^{\star} \sigma^{*}$, 5 exs., Pieria, Olympos, Prionia, $1200 \mathrm{~m}$, beech forest, litter sifted, 29.V.2004, leg. Schuh (cAss); $3 \sigma^{\star} \sigma^{\star}, 3$ 우 우, Makedhonía, Olympos, NW Stavros, 4007'N, 22²6'E, $1500 \mathrm{~m}$, 7.IV.1998, leg. Assing \& Wunderle (cAss, cWun); $1 \sigma^{\star}$, Olympos, 900 m, beech forest, 18.VI.1976, leg. Casale (cZan); 1 o $^{\star}, 1$ ㅇ, Ossa Oros, $1000 \mathrm{~m}$, 18.V.1997, leg. Wolf (MNB); 1 ex., Ossa Oros, 1000 m, 17.VII.1976, leg. Casale (cZan); 1 ex., Thessalía, Pílion, N Tsangaridha, $550 \mathrm{~m}$, Platanus trunk, 9.IV.2004, leg. Vít (cAss); 3 ๙ $^{\star}, 2$ 우 우, N Larissa, Kato Olympos, E Kalipefki, $39^{\circ} 58^{\prime} \mathrm{N}, 2^{\circ} 29^{\prime} \mathrm{E}, 1450 \mathrm{~m}$, 6.IV.1998, leg. Assing, Schülke \& Wunderle (cAss, cWun, MNB); 2 o $^{\top}$, same data, but $1580 \mathrm{~m}, 12$. IV.1998, leg. Assing \& Schülke (cAss, MNB).

Diagnosis: Coloration usually reddish. Hind wings either fully developed or of somewhat reduced length (submacropterous morph).

$0^{\star}$ : median lobe of aedeagus (Figs 29-34, 37-39) 0.40$0.43 \mathrm{~mm}$ long; ventral process nearly straight in lateral view, apically distinctly truncate in lateral view and convex in ventral view; internal sac with pair of relatively large apical spines and with curved apico-ventral filiform structure ("fs" in Fig. 39); apical lobe of paramere (Fig. 35) nearly three-fourths as long as basal portion; 


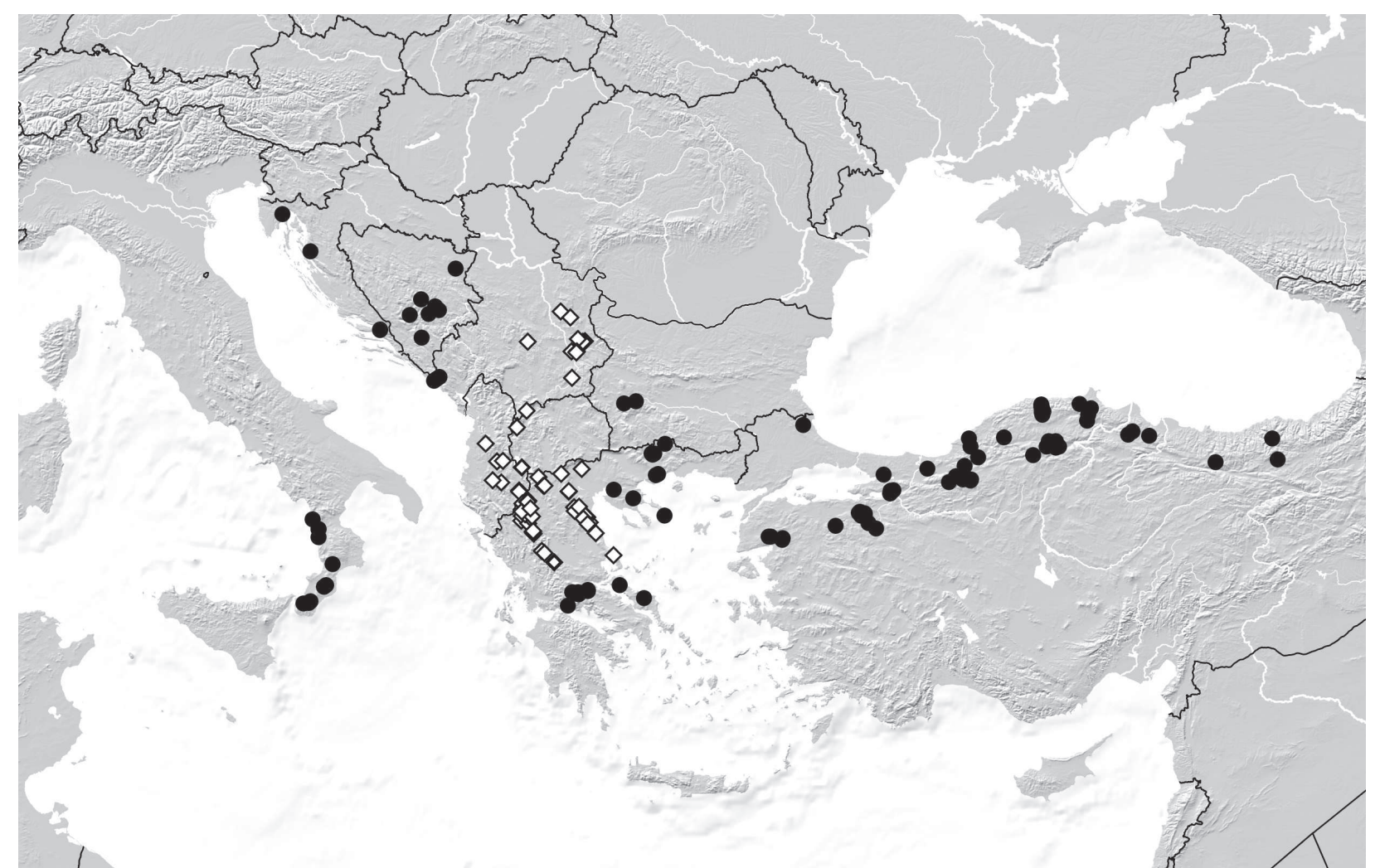

Map 5: Distributions of Oxypoda nova (black circles) and O. kuehnelti (white diamonds) in the East Mediterranean, based on revised records.

basal portion of paramere (Fig. 35) with dark structure of subcircular to trapezoid shape ("ds" in Fig. 35).

+ : spermatheca (Fig. 36) of variable shape, with bellshaped apical cuticular invagination.

Distribution and natural history: The revised distribution of O. kuehnelti is continuous and confined to the Balkans from Serbia in the north across Albania and Macedonia to the south of mainland Greece, where it borders on the ranges of $O$. nova and $O$. rectacia (Maps 1,5 ).

The examined material was sifted from leaf litter, roots, moss, and hollow tree trunks in various forest (deciduous, coniferous, mixed) habitats, The altitudes range from 400 to $1880 \mathrm{~m}$. The specimens were found in April (15 records), May (20), June (12), and July (13).

\section{Oxypoda (Baeoglena) rectacia spec. nov. urn:Isid:zoobank.org:act:8E5A6F28-9AD3-474E-99F8-9CBA0F85C4DC (Figs 40-54, Maps 1, 6)}

Type material: Holotype o*: "Greece: Karpathos [8],

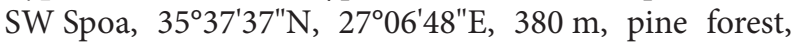
litter \& grass sift., 25.XII.2015, V. Assing / Holotypus o Oxypoda rectacia sp. n. det. V. Assing 2018” (cAss).

Paratypes: Greece: Karpathos: $10^{\star}$ : "Greece: Karpathos [7], S Spoa, 3536' $28^{\prime \prime} \mathrm{N}, 27^{\circ} 08^{\prime} 43^{\prime \prime} \mathrm{E}, 250 \mathrm{~m}$, stream valley, sifted, 25.XII.2015, V. Assing” (cAss); 2 우 우 “Greece:

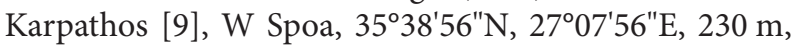
stream valley, sifted, 25.XII.2015, V. Assing” (cAss); 1 ơ:

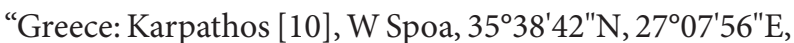
$210 \mathrm{~m}$, stream valley, sifted, 25.XII.2015, V. Assing"

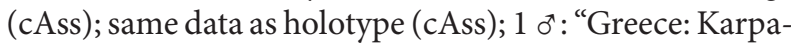

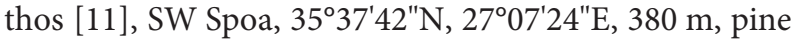
forest, litter \& grass sift., 25.XII.2015, V. Assing" (cAss); $4 o^{\star} o^{\star}, 9$ 우 우 : "Greece: Karpathos [21], 3 km SSW Spoa, $35^{\circ} 36^{\prime} 42^{\prime \prime} \mathrm{N}, 27^{\circ} 08^{\prime} 08^{\prime \prime} \mathrm{E}, 760 \mathrm{~m}$, pine litter, 30.XII.2015, V. Assing” (cAss); 1 o", 1 ㅇ: "Greece: Karpathos [22],

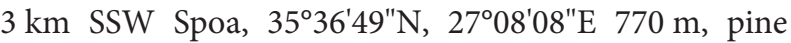
litter, 30.XII.2015, V. Assing" (cAss). Crete: 1 ex., "GR Crete [26], S Anogia, Psiloritis, 35²14'02"N, 2453'13"E, $1280 \mathrm{~m}$, sifted, 2.IV.2012, V. Assing" (cAss); 1 ex.:

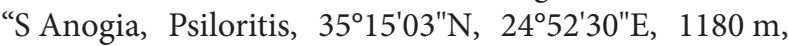
rocky N-slope with oak, litter and grass near snow sifted, 4.IV.2012, leg. Assing" (cAss); $10^{\text {* }}$ : "Kouloukonas, E Perama, $35^{\circ} 22^{\prime} 56^{\prime \prime} \mathrm{N}, 24^{\circ} 50^{\prime} 00^{\prime \prime} \mathrm{E}, 370 \mathrm{~m}$, calcareous $\mathrm{N}$-slope with secondary forest, litter sifted, 17.IV.2014, leg. Assing" (cAss); 1 \%: "GR - Crete [15], S Kissamos, NE Sirikari, 35²4'48"N, 2339'04"E, 500 m, soil washing, 19.III.2018, V. Assing” (cAss); 1 \%: same data, but "[15a] ... dead chestnut ..." (cAss); 2 ㅇ : “GR - Crete [24a],

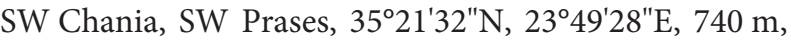
litter sifted, 20.III.2018, V. Assing” (cAss); 1 \%: “GR Crete [51], SE Kandanos, 35¹9'18"N, 2345'41"E, 690 m, old oak tree, soil washing, 25.III.2018, V. Assing" (cAss);

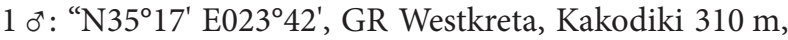
Meybohm 16.3.2001" (cAss); 1 ㅇ: “24.3.86 Westkreta, Samaria-Schlucht, leg. H. Meybohm” (cAss); 1 ㅊ, 3 우 우 "GR - Crete [4], S Kissamos, NW Elos, Limni, 35²2'16"N, 2337'55"E, 580 m, old Platanus, 23.XII.2018, V. Assing” 
(cAss); 1 \%: "GR - Crete [14], ESE Rethimno, W Arkadi M. $35^{\circ} 18^{\prime} 30^{\prime \prime} \mathrm{N}, 24^{\circ} 36^{\prime} 43^{\prime \prime E}, 500 \mathrm{~m}$, soil washing, 26.XII.2018, V. Assing" (cAss); $1 \mathrm{o}^{\text {*: }}$ "GR-Crete [15],

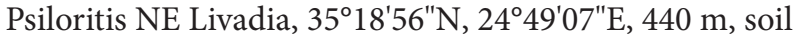
washing near stream, 26.XII.2018, V. Assing" (cAss); $10^{\star}$ : "GR - Crete [25], NE Lassithi Plateau, 35²12'45"N, $25^{\circ} 31^{\prime} 53^{\prime E} 850 \mathrm{~m}$, soil washing, 30.XII.2018, V. Assing”

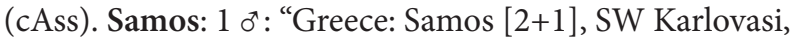
Oros Kerkis: NE-slope, 670 m, 37²4'14"N, 26³9'04"E,

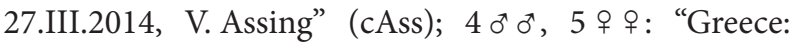
Samos [16+1], Oros Kerkis: NW-slope, E Kalithea, $37^{\circ} 44^{\prime} 36^{\prime \prime} \mathrm{N}, 26^{\circ} 37^{\prime} 02^{\prime \prime E}, 580 \mathrm{~m}, 1 . I V .2014$, V. Assing"

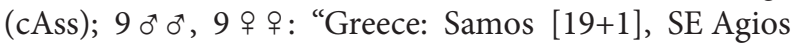
Konstantinos, $37^{\circ} 46^{\prime} 37^{\prime \prime} \mathrm{N}, 26^{\circ} 48^{\prime} 53^{\prime \prime E}, 790$ m, forest litter, 2.IV.2014, V. Assing" (cAss, MNB); $50^{\star} 0^{\star}, 5$ ㅇ 우 : “Greece: Samos [20+1], SE Agios Konstantinos, 3746'41"N, $26^{\circ} 48^{\prime} 28^{\prime \prime E}, 930 \mathrm{~m}$, forest litter, 2.IV.2014, V. Assing" (cAss, MNB); 17 ơ ơ 22 우 우 “Greece: Samos [33b+1], Or. Ambelos: N-slope, 3746'33"N 2648'39"E, 940 m, pine litter sifted, 5.IV. 2014, V. Assing" (cAss, MNB); 1 우 "GREECE: Samos [2], Oros Kerkis: Prof. Ilias, 37²43'43"N, $26^{\circ} 38^{\prime} 03^{\prime E} \mathrm{E}, 1210 \mathrm{~m}$, sifted, 7.IV.2017, V. Assing” (cAss); $60^{\top} o^{\top}, 7$ 우 우: “GREECE: Samos [4a], Oros Ambelos: $\mathrm{N}$-slope, 3746'33"N, 26²48'39"E, 940 m, sifted, 8.IV.2017, V. Assing" (cAss); $30^{\star} o^{\star}, 7$ 우 일 same data, but “[4b]" (cAss); 6 ㅈ $\sigma^{\star}, 10$ 우 우: same data, but “[4d] ... 15.IV.2017” (cAss, MNB); 5 o $^{\star}, 2$ 우 오 : same data, but “[4e]” (cAss); 1 \%: "GREECE: Samos [30], Oros Kerkis, NW-slope, $37^{\circ} 44^{\prime} 35^{\prime \prime N}, 26^{\circ} 37^{\prime} 02^{\prime \prime E}, 630 \mathrm{~m}$, Platanus sifted, 16.IV. 2017, V. Assing" (cAss); 1 ㅇ: "GREECE: Samos [31], Oros Ambelos: N-slope, 37²7'13"N, 2648'03"E, 830 m, litter sifted, 17.IV.2017, V. Assing” (cAss); 1 : "GR - Samos, s/w Kosmadei, ca. 560 m, N3745'26"E26²9'31"E, 30. IV.-1.V.2003, leg. Meybohm/Brachat" (cAss). Ikaría: $10^{\top}$ : "GREECE: Ikaría [7], S Evdilos, pass env., 37³5'20"N, $26^{\circ} 10^{\prime} 22^{\prime \prime E}, 570 \mathrm{~m}$, sifted, 10.IV.2017, V. Assing" (cAss); $20^{\star} 0^{\star}, 3$ ㅇ ㅇ: "GREECE: Ikaría [14], SE Evdilos, $37^{\circ} 35^{\prime} 46^{\prime \prime} \mathrm{N}, 26^{\circ} 12^{\prime} 51^{\prime \prime E}, 780 \mathrm{~m}$, debris \& grass sifted, 11. IV.2017, V. Assing” (cAss); 1 o", 2 ㅇ 우: same data, but “14.

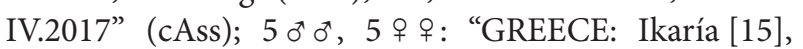
SE Evdilos, 37³6'00"N, 2612'34"E, 670 m, moist litter sifted, 11.IV.2017, V. Assing” (cAss); 2 우 우 “GREECE: Ikaría [20], NNE Pezi, 37³5'11"N, 2604'15"E, 570 m,

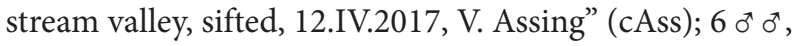
6 우 ㅇ: "GREECE: Ikaría [23], N Agios Kirikos, 37³7'49"N, $26^{\circ} 16^{\prime} 12$ "E, 790 m, phrygana sifted, 13.IV.2017, V. Assing” (cAss); 1 ㅇ: "GREECE: Ikaría [24], S Monikampi, $37^{\circ} 38^{\prime} 24^{\prime \prime} \mathrm{N}, 26^{\circ} 16^{\prime} 10^{\prime} \mathrm{E}, 560 \mathrm{~m}$, forest litter sifted, 13. IV.2017, V. Assing" (cAss). Greece: mainland: $3 o^{-1} o^{-1}, 1$ : "GR. Fthiotis, 1100 m, 1, Oros Kallidromo, SSE Lamia, Abies wood, $38^{\circ} 44^{\prime} 01 \mathrm{~N}, 22^{\circ} 33^{\prime} 32 \mathrm{E}, 02 . \mathrm{IV} .2001$, V. Assing” (cAss); 2 우 우: same data, but leg. Wunderle (cWun); 1 우 "GR. Fthiotis, 940 m, 2, Oros Kallidromo, SSE Lamia, Abies wood, $38^{\circ} 45^{\prime} 29 \mathrm{~N}, 22^{\circ} 28^{\prime} 02 \mathrm{E}, 02 . \mathrm{IV} .2001$, V. Assing” (cAss); 1 ex.: same data, but leg. Wunderle (cWun); 5 o $0^{*}$, 1 ㅇ: "GR. Fthiotis, 940 m, 11, Oros Kallidromo, SSE Lamia, Abies wood, $38^{\circ} 45^{\prime} 29 \mathrm{~N}, 22^{\circ} 28^{\prime} 02 \mathrm{E}, 06 . I V .2001$, V. Assing" (cAss); 18 exs.: same data, but leg. Wunderle
(cWun); $5 o^{\star} o^{\star}, 4$ ㅇ : "GR. Voiotia, 900 m, 6, Oros Elikonas, NE Kiriaki, Abies wood, $38^{\circ} 22^{\prime} 40 \mathrm{~N}, 22^{\circ} 49^{\prime} 16 \mathrm{E}, 04 . \mathrm{IV}$. 2001, V. Assing” (cAss); 6 exs.: same data, but leg. Wunderle (cWun). Evvoia: 1 o $^{*}$ : "GR. N-Evvoia, 19, Oros Lichas, $525 \mathrm{~m}$, Pinus wood, $38^{\circ} 51^{\prime} 11 \mathrm{~N}, 22^{\circ} 54^{\prime} 30 \mathrm{E}, 08$. IV.2001, V. Assing” (cAss). Pelopónnisos: $10^{\star}:$ :N3705'13 E22 ${ }^{\circ} 16^{\prime} 44$, GR Peloponnes (43), Taygetos ö Pass 1100 m, Meybohm 17.4.2018” (cAss); 1 ơ, 1 우: “N3708'28 E22 ${ }^{\circ} 15^{\prime} 56$, GR Peloponnes 23.4.2017, Taygetos s Neochori 1350 m, leg. Brachat \& Meybohm" (cAss); 1 ex., "GR, Peloponnes, Taygetos, Straße zum Prof. Ilias, 11-1400 m, 16.6.96, P. Wunderle" (cWun); 6 exs.: "GR, Peloponnes, Parnon-geb., 1100-1450 m, Pinus, Abies, 13.6.96,

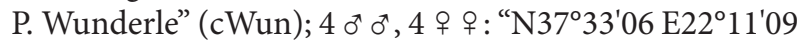
(25), GR Peloponnes 20.4.2017, Ditiko Menalo 1280 m, leg. Brachat \& Meybohm” (cAss); 1 ơ, 1 i : “N37³9'20 E22 ${ }^{\circ} 09^{\prime} 00$, GR Peloponnes, Ditiko Melano [sic] $1050 \mathrm{~m}$, 20.4.2017, leg. Brachat \& Meybohm" (cAss); $10^{\star}, 1$ i : “N37²40'28 E22 ${ }^{\circ} 13^{\prime}$ 04, GR Peloponnes, Mt. Melano [sic] $1470 \mathrm{~m}, 20.4 .2017$, leg. Brachat \& Meybohm" (cAss); $1 \mathrm{o}^{\top}$, 1 ㅇ: Menalo, 37 $41^{\prime} 23^{\prime \prime} \mathrm{N}, 22^{\circ} 12^{\prime} 45^{\prime \prime} \mathrm{E}, 1250 \mathrm{~m}, 19 . \mathrm{IV} .2017$, leg. Brachat \& Meybohm (cAss); 3 우: Ditiko Menalo, $37^{\circ} 36^{\prime} 02^{\prime \prime N}, 22^{\circ} 09^{\prime} 37^{\prime \prime E}, 1230 \mathrm{~m}, 20 . I V .2017$, leg. Brachat

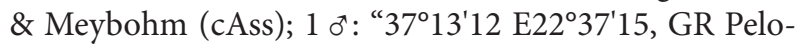
ponnes 22.4.2017, Parnon $1450 \mathrm{~m}$, leg. Brachat \& Meybohm" (cAss); 1 đ , 3 우: “GR. Pelopónnisos, Aroania-Geb., $1450 \mathrm{~m}, 10 \mathrm{~km}$ E Kalavrita, $38^{\circ} 01^{\prime} 02 \mathrm{~N}$, $22^{\circ} 10^{\prime} 21 \mathrm{E}, 29 . \mathrm{III} .1997, \mathrm{~V}$. Assing” (cAss); 4 o $^{\star x}, 9$ 우 우: "GR. Pelopónnisos, Erimanthos, Abies, oberh. Kalendzi, $1200 \mathrm{~m}, \quad 37^{\circ} 57^{\prime} 02 \mathrm{~N}, 21^{\circ} 46^{\prime} 34 \mathrm{E}, 1 . I V .1997$, V. Assing”

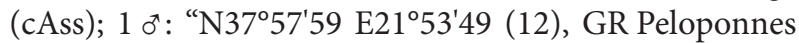
16.4.2017, Erimanthos Vlasia $1150 \mathrm{~m}$, leg. Brachat \&

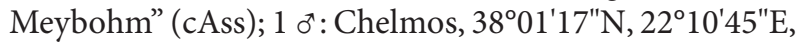
1550 m, 13.IV.2017, leg. Brachat \& Meybohm (cAss); 1 우

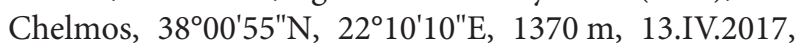
leg. Brachat \& Meybohm (cAss); 1 : Erimanthos, Kalen-

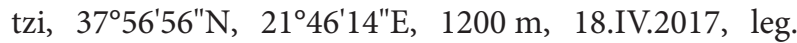
Brachat \& Meybohm (cAss); 5 đ` $0^{\star}, 2$ 우 우 : “GR. Pelopónnisos, Killini-Gebirge, $1300 \mathrm{~m}$, S Trikalon, N-Hang, 375'이, 22²7'27E, 31.III.1997 V. Assing" (cAss); $4 \sigma^{\top} o^{\top}, 4$ ㅇ ㅇ, 9 exs.: "GR. Nord-Peloponnes, Killini-geb., Ano Trikala, 1450 m, Pinusstreu, 7.6.1996, P. Wunderle"

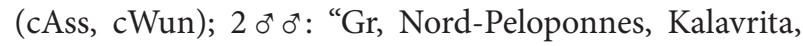
1450 m, Str. zum Xerokambos, 06.06.1996, P. Wunderle" (cWun); $1 \mathrm{o}^{\mathrm{t}}$ : "Greece: Peloponnes, Parnon, $25 \mathrm{~km} \mathrm{NE}$ Sparti, S Katafigio Agrias, Zois, $37^{\circ} 13^{\prime} 03.8^{\prime \prime} \mathrm{N}$, $22^{\circ} 37^{\prime} 19.5^{\prime \prime E}, 1440 \mathrm{~m}$, Abies and Pinus forest, sifted, 23.IV. 2015, leg. M. Schülke” (MNB); 1 ð 3 exs.: “Greece: Peloponnes, Aroania, E Kalavrita, $1440 \mathrm{~m}$, 3801'03"N, $22^{\circ} 10^{\prime} 33 " \mathrm{E}$, Gesiebe, 2.IV.2016, leg. M. Schülke [GR1601]" (MNB); 1 ex.: "Greece: Peloponnes, Killini,

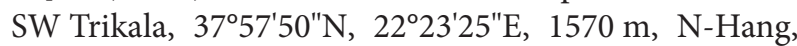
Tannenwald, Gesiebe, 3.IV.2016, leg. M. Schülke [GR1606]” (MNB); 1 ○`, 1 ex.: “Greece: Peloponnes, Killini, SW Trikala, $37^{\circ} 57^{\prime} 40^{\prime \prime} \mathrm{N}, \quad 22^{\circ} 23^{\prime} 16^{\prime \prime E}, 1550 \mathrm{~m}, \quad$ N-Hang, Tannenwald, Gesiebe, 3.IV.2016, leg. M. Schülke [GR1608]” (MNB). Rhodos: 1 ㅇ: "GR Rhodos (9), Ataviros 1000 m, N36² $12^{\prime} 14$ E27 $51^{\prime}$ 04, leg. Meybohm 5.4.2012” 
(cAss). Cyprus: $10^{\star}$ : "Cyprus - Troodos-Geb., SE Prodhromos, Quercus-Best., 1500 m, 01.IV.1995, Assing" (cAss); 1 o : "Cyprus - S Patres, Moniatis, Bachtal, Bachufer, 700 m, 12.IV.1995, Assing" (cAss); 1 : same data, but "02.IV.1995" (cAss); 1 : same data, but "07.IV. 1995” (cAss); 1 ㅇ: "Cyprus - 25 km SW Platres, Kidasi, ca. 300 m, Diarizo-Ufer, Auwald, 11.IV.1995, Assing” (cAss); 1 ㅇ: "GR. Zypern, Kaledonien Wasserfall, 15.III. 1994, leg. H. Meybohm” (cAss); 1 \%: “GR. Zypern, Neo Chorion, 19.III.1994, leg. H. Meybohm” (cAss); 2 우 ㅇ․ "GR. Zypern, Latchi, 09.III.1994, leg. H. Meybohm"

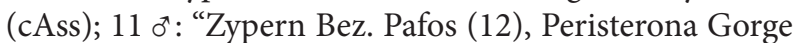
270 m, N3459'39 E32 38'56, 1. Meybohm, 23.IV.2015” (cAss); 2 ๙ $^{\star}$, 5 우 우 : “Zypern Paphos (3), Gefyra Kelefou $430 \mathrm{~m}, 22.3 .2010$ 1. Meybohm, N3453'19 E32 44'54” (cAss); 1 : “N345ㄴ'55 E032 52'13, Zypern 08/8, Troodos 1550 m, leg. Lompe 9.4.2008” (cAss); 5 exs.: "Cyprus occ., Peyia, ca. 11 km NW Paphos, 34.857380N, 32.364704E, sift under Mimosa bushes, 19.-27.III.2018, leg. A. Pütz" (cPüt, cAss); 1 ex.: "Chypre 20.VII.77, Baths of Aprodite, C. Besuchet" (MHNG); 6 exs.: "Chypre 1200 m. V. des Cèdres, 15.VII.77, C. Besuchet” (MHNG, cAss); 1 ex.: "Chypre 12.VII.77, s/ Ayia 950 m. C. Besuchet" (MHNG). Turkey: $1 o^{\star}$, 6 exs.: "Turquie Izmir, Bozdagh, $1200 \mathrm{~m}$, 29.IV.75, Besuchet Löbl” (MHNG, cAss); 1 ex.: "TmolosGbg., [= Boz Dağları] / Lydien. / West-Kleinasien. / Weirather, / Innsbruck / ex coll. Scheerpeltz / Cotypus Oxypoda phrygica O. Scheerpeltz" (NHMW); $2 o^{\star} o^{*}, 1$ i : “N38³2'51 E02725'14 (20), Türkei Manisa Sipil Dagi Milli Parki, 1250 m, 25.4.2006, 1. Brachat \& Meybohm" (cAss); 1 ㅇ: “TR Prov.: Manisa (21), Umgeb. Manisa, Nat. Park Sipil Dagi, 11080 m [sic], N38³2'32”, E2727'44”, leg. Meybohm \& Brachat” (cAss); 2 우 : “TR [8] - Aydın, $15 \mathrm{~km}$ NNE Aydın, Imambaba T., below peak, $1600 \mathrm{~m}$, $37^{\circ} 57^{\prime} 16 \mathrm{~N}, 27^{\circ} 53^{\prime} 55 \mathrm{E}, 5 . I V .2006$, V. Assing” (cAss); $1 \mathrm{o}^{\text {t: }}$ : "TR [9] - Aydın, $15 \mathrm{~km}$ NNE Aydın, Imambaba T., $1530 \mathrm{~m}, \quad 37^{\circ} 57^{\prime} 18 \mathrm{~N}, 27^{\circ} 54^{\prime} 08 \mathrm{E}, 5 . \mathrm{IV} .2006$, V. Assing"

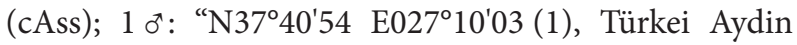
Dilek Dagi, Kanyon 70-370 m, 16.4.2006, 1. Brachat \&

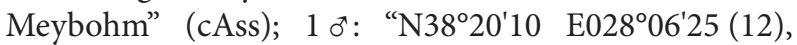
Türkei Aydin Bozdag, 1300-1560 m 21.4.2006, 1. Brachat

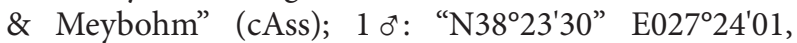
Türkei Izmir Nif Dagi, 1010 m 23.4.2006, 1. Brachat \& Meybohm” (cAss); 2 o $^{\star}$ o $^{\star}, 4$ 우 우: “N38 $24^{\circ} 13$ E027 $23^{\prime} 05$ (18), Türkei Izmir Nif Dagi, 1080 m, 24.4.2006, 1. Brachat \& Meybohm” (cAss); 1 o , 1 \%: “TR Prov.: Izmir (16), Umgeb.: Kemalpasa, Nif Dagi, 1010 m, 23.IV.2006, N38 23'30", E2724'2”, leg. Meybohm \& Brachat" (cAss); 2 우: “TR Prov:: Izmir (12), NO Ödemiz, Boz Dag 1560 m, 21.IV.2006, N38²0'11”, E286'26”, leg. Meybohm \& Brachat” (cAss); 1 ơ, 2 ㅇ 우 : “TR. - Muğla, 18, 1690 m, Gölgeli Dağları, $20 \mathrm{~km}$ NE Köyceğiz,above Ağla, $37^{\circ} 02^{\prime} 54 \mathrm{~N}, 28^{\circ} 49^{\prime} 24 \mathrm{E}, 6 . X .2002$, V. Assing” (cAss); 1 ㅇ: “TR. - Muğla, 19,600 m, Gölgeli Dağları, 20 km NE Köyceğiz, below Ağla, 3701'20N, 28 44'27E, 6.X.2002, V. Assing” (cAss); 1 o , 1 \%: “Türkei Umg. Antalya, Straße nach Saklikent, 1000 m, Eichenstreu,

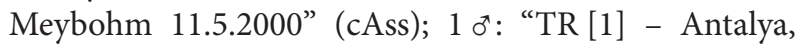

$25 \mathrm{~km}$ ESE Alanya, $900 \mathrm{~m}$, litter of deciduous trees, $36^{\circ} 31^{\prime} 53 \mathrm{~N}, 32^{\circ} 15^{\prime} 42 \mathrm{E}, 23 . X I I .2006$, V. Assing" (cAss); 1 ㅇ: "N3631'44 E32¹4'04 (37), TR Antalya AlanyaTaskent, $30 \mathrm{~km}$ von Alanya $1140 \mathrm{~m}$, Brachat \& Meybohm 25.4.2008” (cAss); 1 \%: “Umg. Akseki, 1300-1500 m / S-Anatolien, lg. H. Franz / Oxypoda praecox Er., det. R. Pace 1988" (NHMW); 1 ex.: "Turquie Antalya, 18 km S.-E. Gazipaşa, 27.IV.78, Besuchet Löbl” (MHNG); $80^{\star} o^{\star}$, 16 ㅇ ㅇ: “TR [6] - Afyon, Sultan Dağları, 15 km SE Çay, $38^{\circ} 31^{\prime} 39^{\prime \prime} \mathrm{N}, 31^{\circ} 11^{\prime} 07^{\prime \prime E}, 1430 \mathrm{~m}$, oak forest, 18.IV.2011, V. Assing” (cAss); 1 ơ , 10 exs.: "Emir-Dagh-Gbg. [= Emir Dağları; approx. $\left.38^{\circ} 50^{\prime} \mathrm{N}, 31^{\circ} 15^{\prime} \mathrm{E}\right]$, Asm.c., Galatien-Phrygien. Weirather, Innsbruck / Typus [or Cotypus] Oxypoda phrygica Scheerpeltz / Coll. L. Weirather Innsbruck" (MHNG, NHMW); 4 o o o $^{*} 3$ ㅇ ㅇ: “TR [16] - Konya,

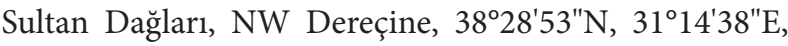
$1320 \mathrm{~m}$, oak for. sifted, 21.IV.2011, V. Assing” (cAss); $3 o^{\top} o^{\star}, \quad 20$ exs.: "N38²9'47 E31 ${ }^{\circ} 11^{\prime} 37$ (23), TR Konya, Sultan Dag, sw Sultandagi, $1730 \mathrm{~m}, 19 . \mathrm{IV} .2008$, leg. Brachat \& Meybohm” (cAss); 1 ㅇ: "N3802'33 E3137'41 (26), TR Konya, Sultan Dag, Destigin, 1540 m, 19.IV. 2008, Brachat \& Meybohm, 20.4.2008" (cAss); 2 exs.: "Turquie Konya, 18 km. S.-O. Beyşehir, 1200 m. 7-V-78,

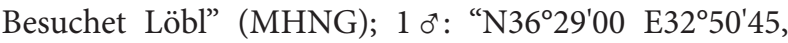
TR Karaman Ermenek, Schlucht s Kazanki 1390 m, Brachat \& Meybohm 22.4.2008” (cAss); 1 o": “TR Mersin, 18, $1400 \mathrm{~m}, \mathrm{~N}$ Pozanti Tekır, cedar wood, 4.V.2002, $37^{\circ} 18 \mathrm{~N}, 34^{\circ} 51 \mathrm{E}$, Meybohm \& Brachat" (cAss); 3 exs.: "TR [4] - Mersin, $970 \mathrm{~m}, 23 \mathrm{~km}$ N Silifke, $36^{\circ} 31^{\prime} 54 \mathrm{~N}, 33^{\circ} 56^{\prime} 18 \mathrm{E}, 18 . I V .2005$, leg. Brachat \& Meybohm” (cAss); 2 exs.: “TR [16] - Mersin, $21 \mathrm{~km}$ N Ermenek, N Anamur, 3654'20N, 3254'26E, 1620 m, 21.IV.2005, Brachat \& Meybohm" (cAss); 3 exs.: "TR [17] - Mersin, $2 \mathrm{~km}$ S Kasanc1, N Anamur, 36 $43^{\prime} 20 \mathrm{~N}$, $32^{\circ} 55^{\prime} 35 \mathrm{E}, 1580 \mathrm{~m}, 21 . I V .2005$, Brachat \& Meybohm"

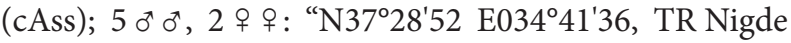
Madenköy E $1200 \mathrm{~m}$ 17.4.2011, Brachat \& Meybohm (9)"; 1 ơ: “TR - Niğde (9), Umg. Çiftehan ö. Maden, 1200 m, 17.IV.2011, N37²8'52, E34º41'36, leg. Meybohm \& Brachat" (cAss); 1 ơ: “TR - Adana (4), s/o Pozanti, Belemedik, 691 m, 27.IV.2011, N37²0'10, E3455'08, leg. Meybohm \& Brachat" (cAss); $10^{\star}, 1$ 우 : N37 $35^{\circ} 23$ E03453'05, TR Adana Kamisli, 1350 m, 18.4.2011, Brachat \& Meybohm (15)" (cAss); 2 exs.: "ostw. Osmaniye/A, 12-1700 m, Asm. VI.68, leg. F. Schubert” (NHMW); 1 ex.: "östl. Osmaniye, $1800 \mathrm{~m}$, Adana / 18.-27.5.70, leg. F. Schubert” (NHMW); 1 ex.: "Asm. Osmaniye, leg. F. Schubert / 5.67" (NHMW); $20^{\star} o^{\star}$, 2 ㅜ: “TR Adana (26), Nur Dag SE Osmaniye, Zorkum $1670 \mathrm{~m} / 36^{\circ} 58^{\prime} 8 \mathrm{~N}, 36^{\circ} 21^{\prime} 39 \mathrm{E}$ (26), 29.4.2004, leg. Brachat \& Meybohm” (cAss); 2 우: “TR [47] - $30 \mathrm{~km} \mathrm{SSW}$ Kahramanmaraş, 660 m, Uzunsöğut, 4.V.2005, 37²23'45N, $36^{\circ} 48^{\prime} 05 \mathrm{E}$, Brachat \& Meybohm” (cAss); 2 우 우 “TR Kahramanmaraş, $14 \mathrm{~km}$ SW Türkoğlu, 37²1'06N, $36^{\circ} 44^{\prime} 22$ E, 850 m, 19.IV.2005 [52], Brachat \& Meybohm" (cAss); 1 \%: “TR. - Antakya [1], 920 m, 19 km S Antakya,

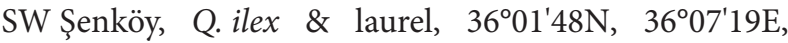
2.IV.2004, leg. V. Assing” (cAss); 1 ex.: “Turkey (Anta- 
kya): Ziyaret Dağı, 22 km S Antakya, SW Şenköy, 940 m, $36^{\circ} 00^{\prime} 32^{\prime \prime} \mathrm{N}, 36^{\circ} 07^{\prime} 13^{\prime \prime} \mathrm{E}$, N slope, oak, beech \& laurel shrubs, sifted, 2.IV.2004, leg. M. Schülke [T04-02]" (MNB); $10^{\star}:$ “TR. - Antakya [13], $920 \mathrm{~m}, 19 \mathrm{~km}$ S Antakya, SW Şenköy, Q. ilex \& laurel, $36^{\circ} 01^{\prime} 48 \mathrm{~N}$, 3607'19E, 5.IV.2004, leg. V. Assing" (cAss); 1 ex.: same data, but leg. Schülke (MNB); $10^{\star}, 4$ 우: “TR. Antakya [11] $1240 \mathrm{~m}, 9 \mathrm{~km}$ SE Iskenderun, $5 \mathrm{~km}$ NE Belen, forest, 36 $31^{\prime} 19 \mathrm{~N}, 36^{\circ} 14^{\prime} 50 \mathrm{E}$, 4.IV.2004, leg. V. Assing” (cAss); 1 ㅇ: "N362 $25^{\prime} 01$ E036 $06^{\prime} 40$, TR Hatay Kizildag, E Madenli $1120 \mathrm{~m}$ 11.4.2009, Brachat \& Meybohm” (cAss); 2 exs.: “TR Antakya (3), Ziyaret Dag, W Sungur $760 \mathrm{~m} / 35^{\circ} 59^{\prime} 34^{\prime \prime} \mathrm{N}, 36^{\circ} 5^{\prime} 18^{\prime \prime} \mathrm{E}, 21.4 .2004$, leg. Brachat \& Meybohm” (cAss); $10^{\star}$ : “N3601'56 E36 07'17, Türkei Hatay Senköy, $920 \mathrm{~m}, 13.4 .2009$, Brachat \& Meybohm (10)” (cAss); 1 ㅇ, “N36³'30,6 E36²'36,2 / TR - Hatay (8), s Harbiye, Umg. Senköy, 730 m, 13.IV.2009, leg. Meybohm \& Brachat" (cAss); 7 exs.: "Turquie Antakya, Kişlak - Şenköy, 800-850 m. 2.V.78, Besuchet Löbl” (MHNG). Syria: $10^{\top}$ : "Syria: 17.IV.2008, Jabal an Nusayriyah, Slunfeh, 544 m, Hlaváč lgt., N35³6.549' E36 $05.775^{\prime \prime}$ (cAss). Lebanon: $20^{\star} \sigma^{\star}, 2$ exs.: "Lebanon, Rayfoun, ca. $33^{\circ} 58^{\prime} \mathrm{N}, 35^{\circ} 42^{\prime} \mathrm{E}$, mix. oak for., $990 \mathrm{~m}$, 31.III.-9.IV.2016, pitfall trap, leg. Reuter" (cFel, cAss); 5 exs.: "Liban 3.IV.75, Hasroun près de Becharré, Besuchet 1500 m" (MHNG); 1 ex.: "Liban 2.IV.75, Les Cédres prés de Becharré, 1950-2000 m, Besuchet" (MHNG); 1 ex.: "Liban 27.III.75, Beit Eddine, Besuchet 900 m." (cAss); 3 exs.: "Liban 26.III.75, Jeita, Besuchet" (MHNG, cAss). Israel: 10 exs.: "Israel: Galilée, Eilon, N. Betzet, 22.IV.1982, Besuchet, Löbl” (MHNG, cAss); 12 exs.: same data, but 20.IV.1982 (MHNG, cAss); 1 ex.: "Israel: Galilée, Mt Meron, 700 m, 26.IV.1982, Besuchet, Löbl” (MHNG); 4 exs.: "Israel: Galilee, Mt. Meron, 900 m, 27.V.73, Löbl" (MHNG).

Etymology: The specific epithet is a noun in apposition composed of the adjective rectus (straight) and the noun acia (thread). It alludes to the straight filiform ventral structure in the internal sac of the aedeagus.

Description: Body length 2.3-3.0 mm; length of forebody 1.1-1.5 mm. Habitus as in Fig. 40. Coloration variable: head reddish to black, usually dark-brown to black; pronotum reddish to blackish-brown with paler margins, mostly reddish to reddish-brown; elytra dark-yellowish to dark-brown; abdomen uniformly reddish to blackish with the margins of the tergites and the apical segments reddish; legs yellow to reddish-yellow; antennae darkreddish to dark-brown with the basal 2-3 antennomeres yellowish to pale-reddish.

Head wedge-shaped; punctation fine and dense; interstices with distinct microreticulation. Eyes flat, approximately as long as postocular portion of head in dorsal view. Antenna approximately $0.6 \mathrm{~mm}$ long; antennomeres IV as long as broad or weakly transverse, $\mathrm{V}-\mathrm{X}$ of gradually increasing width and increasingly transverse, VIII-X nearly twice as broad as long.
Pronotum approximately $1.35-1.40$ times as broad as long and approximately 1.7 times as broad as head; punctation dense, more distinct than that of head; interstices with microreticulation.

Elytra approximately as long as pronotum, or shorter; punctation dense, more distinct than that of pronotum; interstices with superficial microreticulation. Hind wings fully developed. Metatarsomere I longer than the combined length of metatarsomeres II-IV.

Abdomen: punctation fine and dense, nearly as dense on tergite VII as on anterior tergites; microreticulation very shallow, often extensively obsolete, especially on posterior tergites; posterior margin of tergite VII with palisade fringe; posterior margin of tergite VIII strongly convex.

$\mathrm{o}^{*}$ : posterior margin of sternite VIII strongly convex; median lobe of aedeagus (Figs 41-50) approximately $0.30-0.33 \mathrm{~mm}$ long; ventral process straight and apically truncate in lateral view, slender and apically convex in ventral view; crista apicalis very small and sinuate; internal sac with straight filiform apico-ventral structure ("fs" in Fig. 48) and with pair of slender and weakly sclerotized spines; paramere (Fig. 51) 0.45-0.50 mm long; apical lobe weakly dilated basally, approximately three-fourths as long as basal portion.

i: posterior margin of sternite VIII convex; spermatheca (Figs 52-54) with moderately long and rather stout proximal portion and with relatively large bell-shaped apical cuticular invagination.

Intraspecific variation: This species is subject to pronounced intraspecific variation especially of size, coloration, and wing length, but also of other external characters such as punctation and microsculpture.

Comparative notes: Based on the similar general structure of the male and female primary sexual characters, O. rectacia is most closely allied to O. kuehnelti, from which it differs by on average slightly smaller body size, on average darker coloration, and particularly by the smaller median lobe of the aedeagus with a straight filiform apico-ventral structure and the pair of internal spines of different shapes, as well as by the absence of a dark subapical structure of the basal portion of the paramere.

Distribution: The distribution ranges from the Pelopónnisos and the southwest of mainland Greece across Crete, Karpathos, some South Aegean islands, South Anatolia, and Cyprus to the Middle East (Syria, Lebanon, North Israel) (Maps 1, 6).

The habitat requirements are evidently very similar to those of O. fusina, O. nova, and O. kuehnelti. The material was sifted from leaf litter, grass roots, moss, and other debris in various types of forest (deciduous, mixed, coniferous; floodplain) and shrub (phrygana) habitats, as well as on stream banks. The altitudes range from 210 to approximately $2000 \mathrm{~m}$. The specimens were found in March (16 records), April (93), May (8), June (5), July (3), October (2), and December (8). 


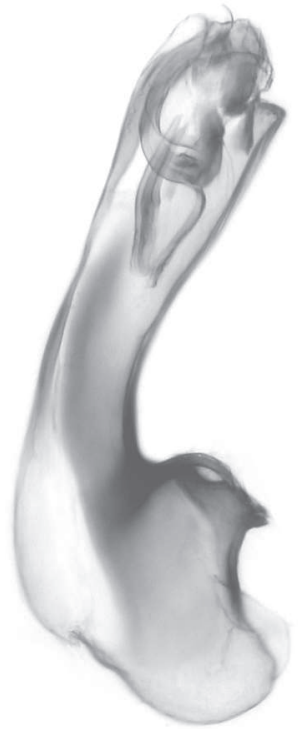

41

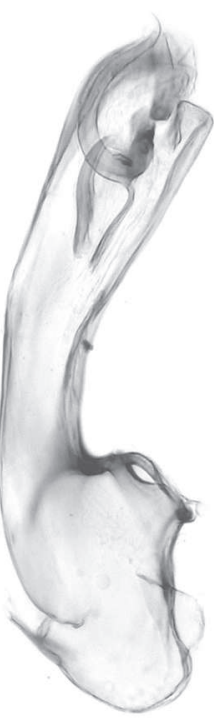

42

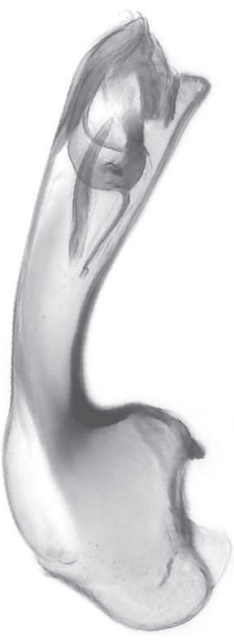

43
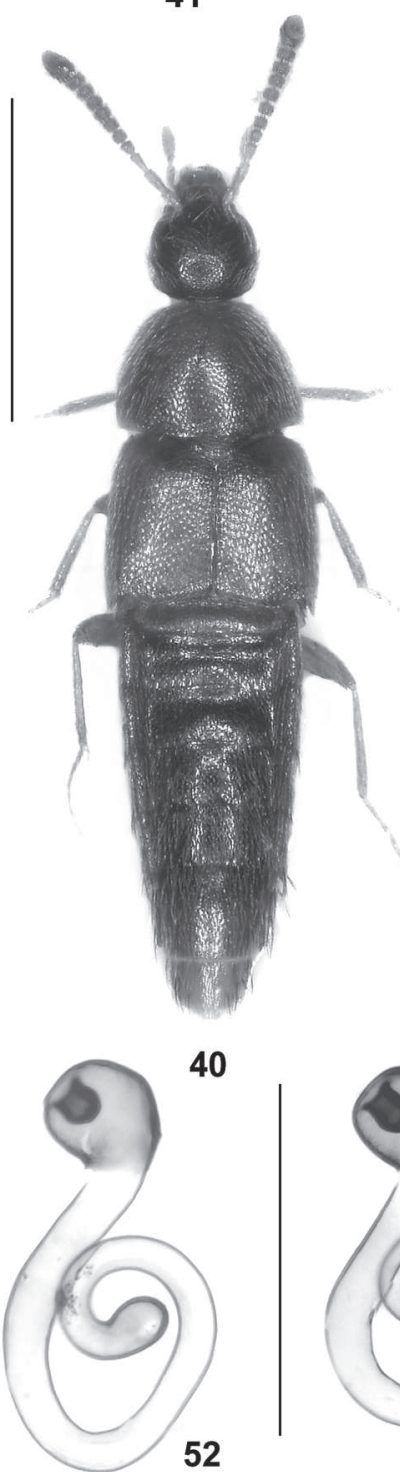

40

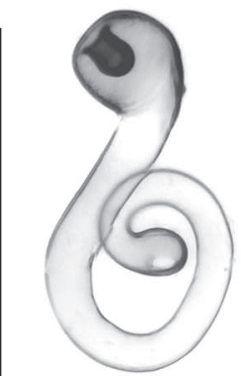

53
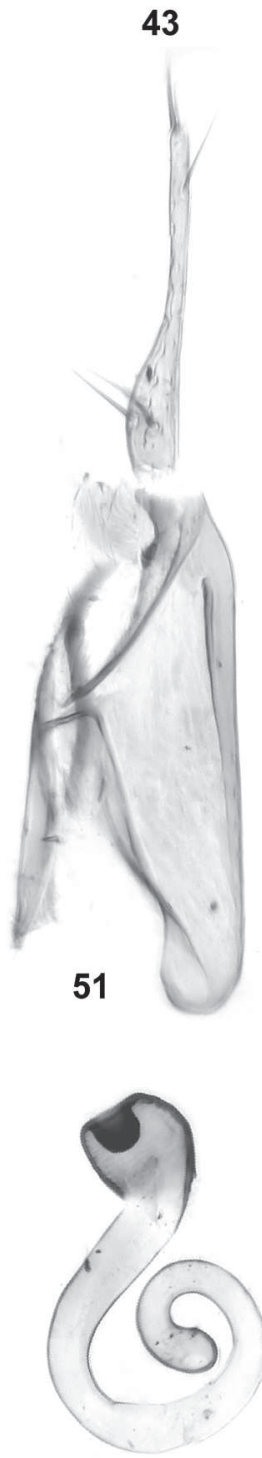

54

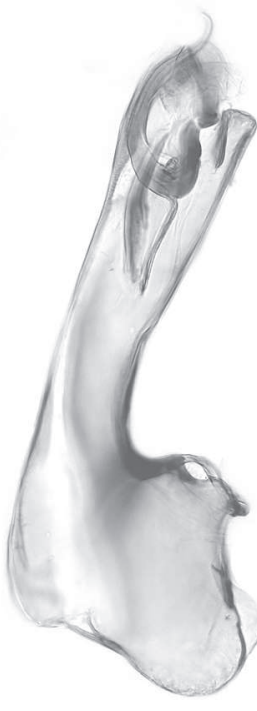

44

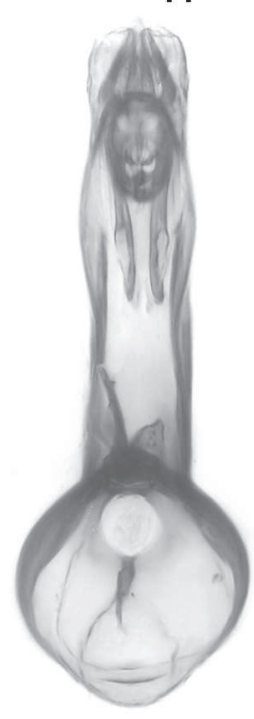

47

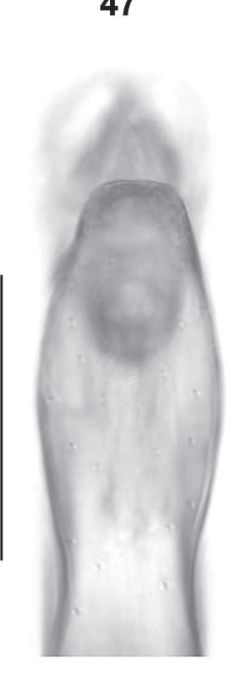

50

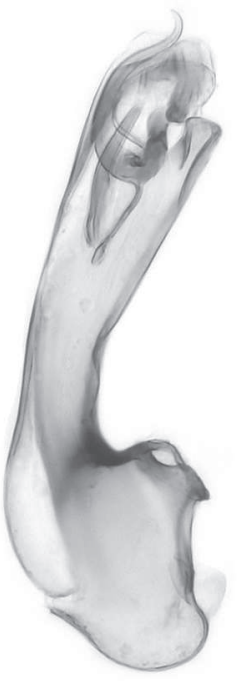

45

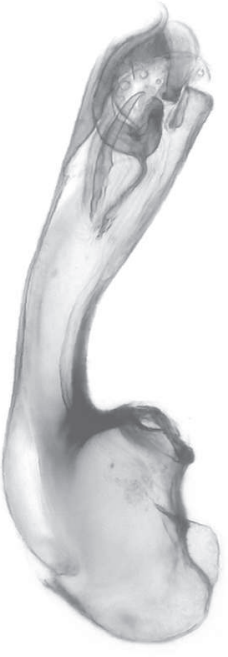

46

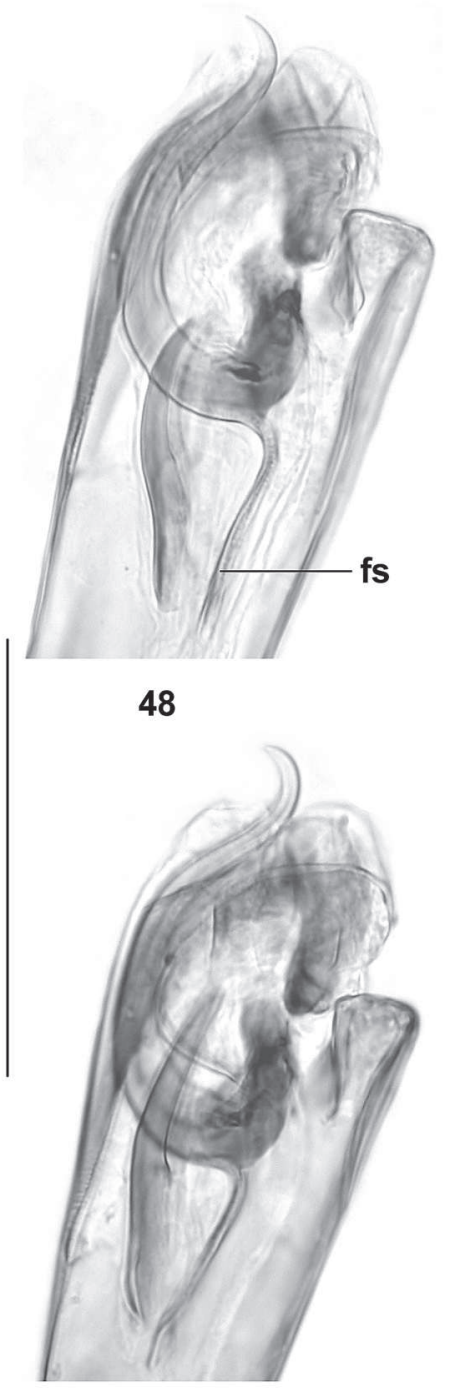

49

Figs 40-54: Oxypoda rectacia from Crete (41-42, 47-48, 50-51, 54), Karpathos (40, 45, 49, 52-53), Ikaría (43), Cyprus (44), and the Pelopónnisos (46): habitus (40); median lobe of aedeagus in lateral and in ventral view (41-47); apex of median lobe of aedeagus in lateral and in ventral view (48-50; "fs" = apico-ventral filiform structure); paramere (51); spermatheca (52-54). Scale bars: 40: $1.0 \mathrm{~mm}$; 41-47, 51-54: $0.2 \mathrm{~mm}$; 48-50: $0.1 \mathrm{~mm}$. 


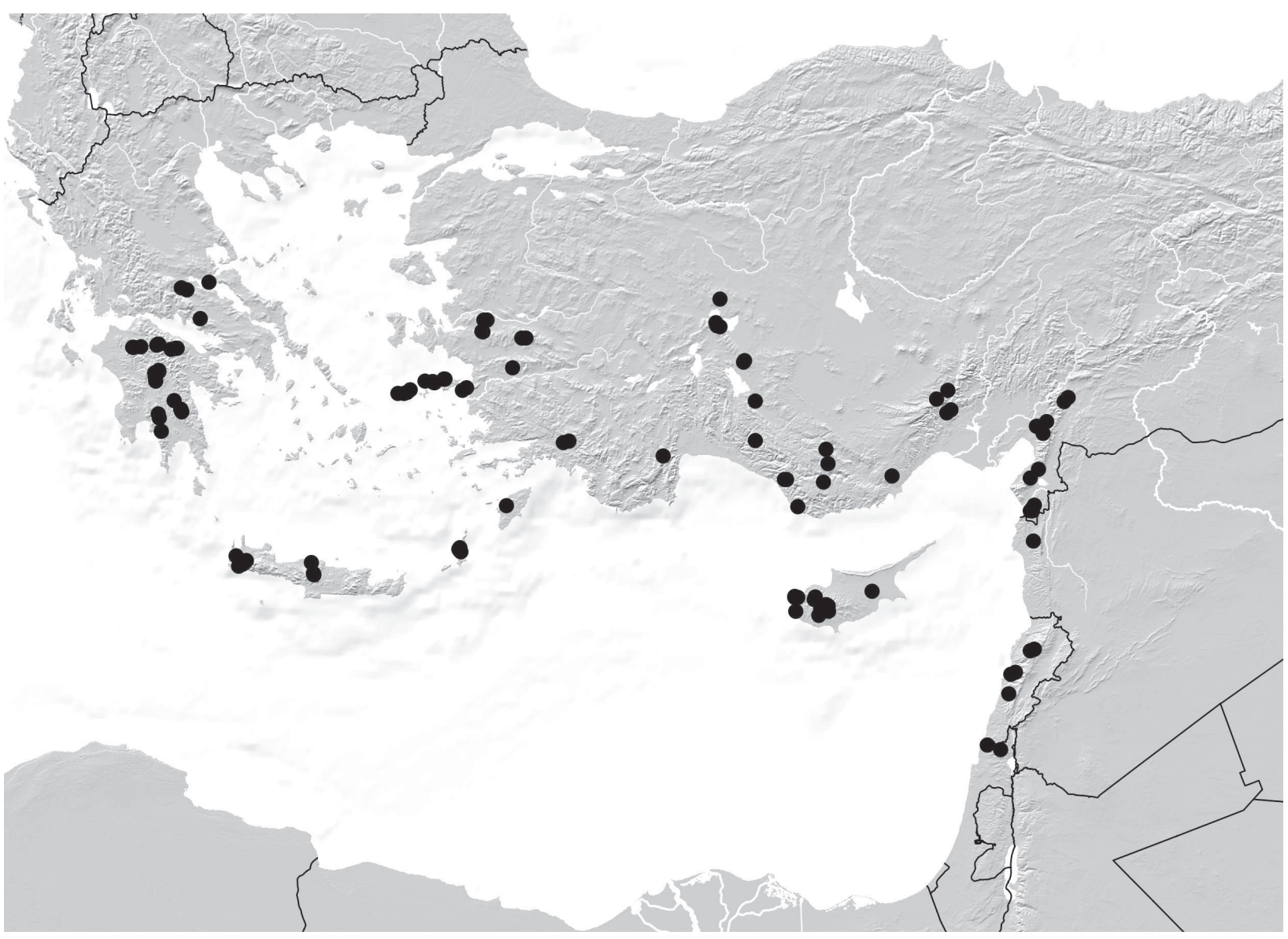

Map 6: Distribution of Oxypoda rectacia in the East Mediterranean, based on revised records.

Oxypoda (Baeoglena) caucasica Bernhauer, 1902 (Figs 55-58, Maps 1, 7)

Oxypoda (Baeoglena) nova Bernhauer, 1902: 180.

Type material examined: Lectotype $\sigma^{\star}$, present designation: "Caucasus, Helenendorf, Reitter. / fusina Rey / vid. Bernh. / caucasica Bh. / ex coll. Skalitzky / Lectotypus o Oxypoda caucasica Bernhauer, desig. V. Assing 2018 / Oxypoda caucasica Bernhauer, det. V. Assing 2018” (NHMW).

Comment: The original description is based on an unspecified number of syntypes from "Caucasus: Helenendorf, Martkopi, Meskisches Gebige [sic], Daghestan, Lenkoran" (Bernhauer 1902). A male syntype from "Helenendorf" is designated as the lectotype.

Material examined: Ukraine: $1 \sigma^{\star}, 1$ ex., Odessa obl., Dobroolexandrivka [“Alexanderhilf”; 4620'N, 30²9'E], leg. Leder (NHMW).

Turkey: Bolu: 1 ơ $^{\star}, 1$ 웅, road Bolu-Yı̆̆ $1 \mathrm{lca}, 40^{\circ} 51^{\prime} \mathrm{N}$, $31^{\circ} 39^{\prime} \mathrm{N}, 1060 \mathrm{~m}, 29 . \mathrm{IV} .2012$, leg. Brachat \& Meybohm

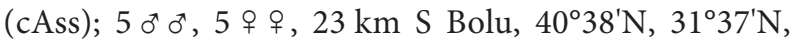
1540 m, 30.IV.2012, leg. Brachat \& Meybohm (cAss); 1 우, Karabük, pass W Eskipazar, $40^{\circ} 58^{\prime} \mathrm{N}, 33^{\circ} 23^{\prime} \mathrm{E}, 1400 \mathrm{~m}$, beech forest, litter sifted, 26.III.2010, leg. Assing (cAss).
Kastamonu: $10^{\star}, 5$ exs. [found together with O. nova], $5 \mathrm{~km}$ S Küre, $1200 \mathrm{~m}, 18$. V.1976, leg. Besuchet \& Löbl (MHNG, cAss). Sinop: 1 ơ $^{\star}, 2$ ㅇ ㅇ, Çangal Dağı, 7-15. VI.1960, leg. Schubert (cAss). Trabzon: 1 \% , ca. $50 \mathrm{~km} \mathrm{~S}$ Trabzon, $20 \mathrm{~km} \mathrm{~S}$ Maçka, Altındere Milli Park, 4040'N, $39^{\circ} 40^{\prime} \mathrm{E}, 1560 \mathrm{~m}$, spruce forest with Rhododendron, sifted, 26.VII.2006, leg. Schülke (MNB). Rize: 1 우, ca. $50 \mathrm{~km}$ S Ardeşen, Çat, 4052' N, 4056'E, $1240 \mathrm{~m}$, Alnus forest, sifted, 3.VIII.2006, leg. Schülke (MNB); 1 ㅇ, ca. $30 \mathrm{~km} \mathrm{SW}$ Hopa, river valley of Çağlayan D., ca. $41^{\circ} 15^{\prime} \mathrm{N}, 41^{\circ} 13^{\prime} \mathrm{E}, 500 \mathrm{~m}, 29 . \mathrm{VI} .1998$, leg. Solodovnikov (cAss). Artvin: 6 exs., Borçka, 1-3.VI.1960, leg. Schubert (cAss); 5 exs., pass between Borçka and Hopa, 700 m, 8.VI.1986, leg. Besuchet, Löbl \& Burckhardt (MHNG, cAss); 1 ex., same locality, 14.V.1967, leg. Besuchet (MHNG); 68 exs., Karkal Dağ1, Pirnalli, 1600 m, 11.VI.1986, leg. Besuchet, Löbl \& Burckhardt (MHNG, cAss); 14 exs., Karkal Dağı, below Pirnalli, 1250 m, 11.VI.1986, leg. Besuchet, Löbl \& Burckhardt (MHNG, cAss); 3 exs., S Artvin, 800 m, 7.VI.1986, leg. Besuchet, Löbl \& Burckhardt (MHNG); 19 exs., S Artvin, 1500 m, 9.VI.1986, leg. Besuchet, Löbl \& Burckhardt (MHNG, cAss); 41 exs., SW Artvin, 1900 m, 9.VI.1986, leg. Besuchet, Löbl \& Burckhardt (MHNG, cAss). Kars: 1 ㅇ, Kars, 1750 m, 17.VI.1986, leg. Besuchet, Löbl \& Burckhardt (MHNG). 
Russia: 1 ex., Krasnodar, 35 km NNE Sochi, Babuk-Aul, $43^{\circ} 53^{\prime} \mathrm{N}, 39^{\circ} 49^{\prime} \mathrm{E}, 560 \mathrm{~m}$, Fagus orientalis and Castanea sativa forest, litter and bark sifted, 11.VII.2011, leg. Assing (cAss); 2 exs., Krasnodar, Temnolesskaia, near Mezmay, 800 m, 8.VI.1999, leg. Smetana (cAss); 25 exs. [partly teneral], Karachayevo-Cherkesskaya Respublika, $13 \mathrm{~km}$ SW Teberda, $43^{\circ} 20^{\prime} \mathrm{N}, 41^{\circ} 40^{\prime} \mathrm{E}$, $1450 \mathrm{~m}$, moist spruce forest with scattered beech, litter, moss, and dead wood sifted, 22.VII.2011, leg. Assing (cAss); 10 exs. [partly teneral], Karachayevo-Cherkesskaya Respublika, $20 \mathrm{~km}$ SW Teberda, above Dombai, $43^{\circ} 17^{\prime} \mathrm{N}, 41^{\circ} 38^{\prime} \mathrm{E}, 1950 \mathrm{~m}$, mixed forest (fir, spruce, beech), leaf litter sifted, 25.VII.2011, leg. Assing (cAss); 1 ex., Karachayevo-Cherkesskaya Respublika, 9 km SW Teberda, Teberdinski range, Baduk river valley, $43^{\circ} 23^{\prime} \mathrm{N}$, $41^{\circ} 40^{\prime} \mathrm{E}, 2000 \mathrm{~m}$, subalpine forest (maple, birch, with rhododendron) near lakeshore, litter sifted, 26.VII.2011, leg. Assing (cAss); 11 exs., Karachayevo-Cherkesskaya Respublika, $9 \mathrm{~km}$ SW Teberda, Teberdinski range, Baduk river valley, $43^{\circ} 23^{\prime} \mathrm{N}, 41^{\circ} 40^{\prime} \mathrm{E}, 2000 \mathrm{~m}$, spruce forest, bark of spruce and maple sifted, 27.VII.2011, leg. Assing (cAss); 1 ex., Krasnaja Poljana, Mt. Atchichkho, 800 m, 26.VI.1989, leg. Arndt (SDEI); 2 exs., Krasnaja Poljana, leg. Roubal (NHMW).

Georgia: 6 exs., Algeti National Park, W Manglisi, $41^{\circ} 42^{\prime} \mathrm{N}, 44^{\circ} 21^{\prime} \mathrm{E}, 1120 \mathrm{~m}, 11 . \mathrm{VII} .2015$, leg. Brachat \& Meybohm (cAss); 6 exs., Algeti National Park, W Manglisi, $41^{\circ} 42^{\prime} \mathrm{N}, 44^{\circ} 19^{\prime} \mathrm{E}, 1110 \mathrm{~m}, 11$. VII.2015, leg. Brachat \& Meybohm (cAss); 1 ex., Algeti National Park, W Manglisi, $41^{\circ} 42^{\prime} \mathrm{N}, 44^{\circ} 18^{\prime} \mathrm{E}, 1210 \mathrm{~m}, 11 . \mathrm{VII} .2015$, leg. Brachat \& Meybohm (cAss); 1 ex., Algeti National Park, Manglisi, $41^{\circ} 42^{\prime} \mathrm{N}, 44^{\circ} 23^{\prime} \mathrm{E}, 1190 \mathrm{~m}, 12 . \mathrm{VII} .2015$, leg. Brachat \& Meybohm (cAss); 10 exs., same data, but 13.VII.2015 (cAss); 1 ex., Central Caucasus, Schatili-Mutso, $42^{\circ} 40^{\prime} \mathrm{N}, 45^{\circ} 11^{\prime} \mathrm{E}, 1390 \mathrm{~m}, 15$. VII.2015, leg. Brachat \& Meybohm (cAss); 5 exs., Central Caucasus, Gudani, 42² $32^{\prime} \mathrm{N}, 44^{\circ} 59^{\prime} \mathrm{E}, 1710 \mathrm{~m}, 18 . \mathrm{VII} .2015$, leg. Brachat \& Meybohm (cAss); 4 exs., Gudani-Zhinvali, $42^{\circ} 27^{\prime} \mathrm{N}, 44^{\circ} 56^{\prime} \mathrm{E}, 1200 \mathrm{~m}, 19 . \mathrm{VII} .2015$, leg. Brachat \& Meybohm (cAss); 8 exs., Gudani-Zhinvali, $42^{\circ} 27^{\prime} \mathrm{N}$, $44^{\circ} 56^{\prime} \mathrm{E}, 1200 \mathrm{~m}, 19 . \mathrm{VII} .2015$, leg. Brachat \& Meybohm (cAss); 1 ex., Shida Kartli, Kvishkheti, $41^{\circ} 58^{\prime} \mathrm{N}, 43^{\circ} 30^{\prime} \mathrm{E}$, $810 \mathrm{~m}$, 12.V.2016, leg. Brachat \& Meybohm (cAss); 6 exs., Shida Kartli, Kvishkheti, $41^{\circ} 57^{\prime} \mathrm{N}, 43^{\circ} 30^{\prime} \mathrm{E}, 790 \mathrm{~m}$, 12.V.2016, leg. Brachat \& Meybohm (cAss); 6 exs., Samtskhe-Javakheti, Timotesubani, $41^{\circ} 49^{\prime} \mathrm{N}, 43^{\circ} 31^{\prime} \mathrm{E}$, $1144 \mathrm{~m}, 13 . \mathrm{V} .2016$, leg. Brachat \& Meybohm (cAss); 3 exs., Samtskhe-Javakheti, Bakuriani, $41^{\circ} 44^{\prime} \mathrm{N}, 43^{\circ} 43^{\prime} \mathrm{E}$, $1770 \mathrm{~m}, 13 . \mathrm{V} .2016$, leg. Brachat \& Meybohm (cAss); 2 exs., Samtskhe-Javakheti, N Abastumani, $41^{\circ} 46^{\prime} \mathrm{N}$, $42^{\circ} 50^{\prime} \mathrm{E}, 1370 \mathrm{~m}, 15 . \mathrm{V} .2016$, leg. Brachat \& Meybohm (cAss); 2 exs., Samtskhe-Javakheti, W Abastumani, $41^{\circ} 45^{\prime} \mathrm{N}, \quad 42^{\circ} 49^{\prime} \mathrm{E}, 1345 \mathrm{~m}, 15 . \mathrm{V} .2016$, leg. Brachat \& Meybohm (cAss); 1 ex., Abastumani, leg. Leder (NHMW); 3 exs., Racha, $4 \mathrm{~km} \mathrm{~N} \mathrm{Nakerala,} 42^{\circ} 24^{\prime} \mathrm{N}$, $43^{\circ} 02^{\prime} \mathrm{E}, 1150 \mathrm{~m}, 18 . \mathrm{V} .2016$, leg. Brachat \& Meybohm (cAss); 8 exs., Shida Kartii, Kvishkheti, $41^{\circ} 57^{\prime} \mathrm{N}$, $43^{\circ} 29^{\prime} \mathrm{E}, 1300 \mathrm{~m}, 24 . V I I .2016$, leg. Meybohm (cAss);
1 ex., Zemo Svaneti, $4 \mathrm{~km} \mathrm{~N}$ Mazeri, $43^{\circ} 06^{\prime} \mathrm{N}, 42^{\circ} 36^{\prime} \mathrm{E}$, 1690 m, 28.VII.2016, leg. Meybohm (cAss); 2 exs., Zemo Svaneti, $2 \mathrm{~km}$ N Ipari, $43^{\circ} 01^{\prime} \mathrm{N}, 42^{\circ} 50^{\prime} \mathrm{E}, 1670 \mathrm{~m}$, 29.VII.2016, leg. Meybohm (cAss); 1 ex., Adjara, Skhalta valley, $41^{\circ} 35^{\prime} \mathrm{N}, 42^{\circ} 22^{\prime} \mathrm{E}, 800 \mathrm{~m}, 22 . \mathrm{VI} .2017$, leg. Brachat \& Meybohm (cAss); 4 exs., Adjara, Skhalta valley, $41^{\circ} 34^{\prime} \mathrm{N}, 42^{\circ} 25^{\prime} \mathrm{E}, 970 \mathrm{~m}, 22 . V I .2017$, leg. Brachat \& Meybohm (cAss); 3 exs., Svaneti, Mazeri, $43^{\circ} 06^{\prime} \mathrm{N}$, $42^{\circ} 36^{\prime} \mathrm{E}, 1660 \mathrm{~m}, 26 . \mathrm{VI} .2017$, leg. Brachat \& Meybohm (cAss); 1 ex., Svaneti, Mestia-Hatsvali, $43^{\circ} 01^{\prime} \mathrm{N}, 42^{\circ} 45^{\prime} \mathrm{E}$, 2350 m, 27.VI.2017, leg. Brachat \& Meybohm (cAss); 4 exs., Svaneti, Mestia-Ushguli, $3 \mathrm{~km} \mathrm{~N}$ Ipari, $43^{\circ} 01^{\prime} \mathrm{N}$, $42^{\circ} 50^{\prime}$ E, 1750 m, 28.VI.2017, leg. Brachat \& Meybohm (cAss); 1 ex., Svaneti, Mestia-Ushguli, Kala, $42^{\circ} 57^{\prime} \mathrm{N}$, $42^{\circ} 53^{\prime} \mathrm{E}, 1680 \mathrm{~m}, 28 . \mathrm{VI} .2017$, leg. Brachat \& Meybohm (cAss); 1 ex., Imereti, Baghdati, $42^{\circ} 01^{\prime} \mathrm{N}, 42^{\circ} 49^{\prime} \mathrm{E}$, $290 \mathrm{~m}, 18 . \mathrm{V} .2018$, leg. Brachat \& Meybohm (cAss); 3 exs., Imereti, S Sairme, $41^{\circ} 51^{\prime} \mathrm{N}, 42^{\circ} 47^{\prime} \mathrm{E}, 1930 \mathrm{~m}$, 19.V.2018, leg. Brachat \& Meybohm (cAss); 1 ex., Imereti, S Sairme, $41^{\circ} 52^{\prime} \mathrm{N}, 42^{\circ} 47^{\prime} \mathrm{E}, 1890 \mathrm{~m}, 19$. V.2018, leg. Brachat \& Meybohm (cAss); 1 ex., Imereti, S Sairme, $41^{\circ} 53^{\prime} \mathrm{N}, 42^{\circ} 46^{\prime} \mathrm{E}, 1420 \mathrm{~m}, 20 . \mathrm{V} .2018$, leg. Brachat \& Meybohm (cAss); 1 ex., Imereti, S Sairme, $41^{\circ} 53^{\prime} \mathrm{N}$, $42^{\circ} 46^{\prime} \mathrm{E}, 1360 \mathrm{~m}, 20$. V.2018, leg. Brachat \& Meybohm (cAss); 6 exs., Racha, E Shovi, $42^{\circ} 41^{\prime} \mathrm{N}, 43^{\circ} 42^{\prime} \mathrm{E}, 1720 \mathrm{~m}$, 28.V.2018, leg. Brachat \& Meybohm (cAss); 3 exs., Racha, E Shovi, $42^{\circ} 41^{\prime} \mathrm{N}, 43^{\circ} 42^{\prime} \mathrm{E}, 1700 \mathrm{~m}, 28 . V .2018$, leg. Brachat \& Meybohm (cAss); 2 exs., Racha, W Glola, $42^{\circ} 42^{\prime} \mathrm{N}, 43^{\circ} 36^{\prime} \mathrm{E}, 1170 \mathrm{~m}, 28 . \mathrm{V} .2018$, leg. Brachat \& Meybohm (cAss); 1 ex., Ananuri Forest, 8.V.2006, leg. Chalandze (cAss); 1 ex., Trialetskiy Khrebet, Bakuriani, 1500-1600 m, 8.VII.1986, leg. Wrase \& Schülke (SDEI); 1 ex., Tbilisi env., Zchneti, $800 \mathrm{~m}$, VI.1987, leg. Wrase \& Schülke (SDEI); 2 exs., $60 \mathrm{~km} \mathrm{~N}$ Tbilisi, Dusheti, 1500 m, 25.IX.1987, leg. Oehlke (SDEI); 3 exs., Khashuri [41 $\left.59^{\circ} \mathrm{N}, 43^{\circ} 35^{\prime} \mathrm{E}\right]$ (NHMW); 1 ex., "Swanetien", leg. Leder (NHMW); 6 exs., Likhskiy Khrebet ["Meskisches Geb."], leg. Leder (NHMW).

Armenia: see AssING \& SCHÜLKE (2019).

Nagorno-Karabakh: see Assing \& SCHÜLKE (2019).

Azerbaijan: 2 exs., Lenkoran, leg. Leder (NHMW); 1 ex., Lerik, leg. Leder (NHMW); 4 exs., Talysh range (MHNG, NHMW).

Iran: Mazandaran: 1 ex., Elburz Mts., Ramsar Co., Eshkatechal, $36^{\circ} 51^{\prime} \mathrm{N}, 50^{\circ} 33^{\prime} \mathrm{E}, 1050 \mathrm{~m}$, small stream, sifted, 6.VI.2008, leg. Pütz (cAss); 1 ex., Elburz Mts., Babol Co., $2 \mathrm{~km}$ SW Firuz Jah, $36^{\circ} 11^{\prime} \mathrm{N}, 52^{\circ} 39^{\prime} \mathrm{E}, 840 \mathrm{~m}$, small stream, 31.V.2008, leg. Pütz (cAss); 2 exs., Elburz Mts., Tonekabon Co., $4.5 \mathrm{~km}$ SW Khanian, Sehezar forest, $36^{\circ} 33^{\prime} \mathrm{N}, 50^{\circ} 50^{\prime} \mathrm{E}, 940 \mathrm{~m}$, small stream, litter and debris sifted, 5.VI.2008, leg. Pütz (cAss); 4 exs., Elburz Mts., Sari Co., Mohammabad, NE Sangdeh, $36^{\circ} 04^{\prime} \mathrm{N}, 53^{\circ} 10^{\prime} \mathrm{E}$, $1530 \mathrm{~m}$, beech forest, litter sifted, 29.V.2008, leg. Pütz (cAss). 1 ex., Elburz Mts., Sari Co., Mohammabad, E Qolqol, 36 $6^{\circ} 10^{\prime} \mathrm{N}, 53^{\circ} 10^{\prime} \mathrm{E}, 920 \mathrm{~m}$, litter sifted, 29.V.2008, leg. Pütz (cAss); 1 ex., Elburz Mts., Chalus Co., SE Abbasabad, $36^{\circ} 39^{\prime} \mathrm{N}, 51^{\circ} 10^{\prime} \mathrm{E}, 280 \mathrm{~m}$, leaf litter and debris sifted, 4.VI.2008, leg. Pütz (MNB); 3 exs., Taquiabad, 
24.VII.1977, leg. Huber (MHNG). Gilan: 1 ex., Elburz Mts., Rasht Co., 29 km S Rasht, $37^{\circ} 01^{\prime} \mathrm{N}, 49^{\circ} 37^{\prime} \mathrm{E}, 140 \mathrm{~m}$, sifted, 7.VI.2008, leg. Pütz (cAss).

Locality ambiguous, not identified, or not specified: 1 ex., "Armen. Geb., leg. Leder (NHMW); 6 exs., "Kaukas", "Caucasus", etc., leg. Leder, Reitter, etc. (NHMW); 1 ex., "Kaukas, ... Urwald bei Tcharsach", 1.V.1875, leg. Leder (MHNG).

Diagnosis: Winged species of mostly dark and more rarely reddish coloration.

$0^{*}$ : median lobe of aedeagus (Figs 55-56) approximately $0.35 \mathrm{~mm}$ long; ventral process weakly curved in lateral view, apically truncate in lateral view and convex in ventral view; crista apicalis long and broad; internal sac with pair of fork-shaped apical structures (lateral view) and with a weakly curved and weakly sclerotized flagellum; apical portion of paramere (Fig. 57) relatively short, barely half as long as basal portion, rather weakly dilated basally.

ㅇ: spermatheca (Fig. 58) with minute apical cuticular invagination.

Distribution and natural history: The distribution of O. caucasica ranges from South Ukraine and Northwest Anatolia across North Anatolia, the Greater Caucasus, and the Lesser Caucasus (including adjacent regions) to North Iran (Maps 1, 7). The record from Germany in Schülke \& SMetana (2015) is based on Lohse (1989: 231) "nach Angaben von L. Zerche", who reports a male from the environs of Gotha collected in 1867.
This specimen was clearly either mislabelled or misidentified, as can be inferred from the revised distribution of O. caucasica.

The examined material was sifted from leaf litter, roots, moss, and bark in various forest (deciduous, coniferous, mixed) habitats and near streams. The altitudes range from 140 to $2350 \mathrm{~m}$. The specimens were found in March (one record), April (2), May (26), June (27), July (37), August (1), and September (1).

\section{Oxypoda (Baeoglena) derecta spec. nov. urn:Isid:zoobank.org:act:4C73E05F-20F7-4697-A4BA-D9E030B28A27 (Figs 59-67, Maps 1, 7)}

Type material: Holotype ơ: "RU [1] - W-Caucasus, $35 \mathrm{~km}$ NNE Sochi, Babuk-Aul, $560 \mathrm{~m}$, forest litter, 435' $26^{\prime \prime N}$, 3949'11"E, 11.VII.2011, V. Assing / Holotypus o Oxypoda derecta sp. n. det. V. Assing 2018” (cAss). Paratypes: $2 \sigma^{\star} \sigma^{\star}, 6$ 우 우: same data as holotype (cAss); $2 \sigma^{\star} \sigma^{*}, 3$ ㅇ 우: "RU [4] - W-Caucasus, $40 \mathrm{~km}$ NNE Sochi, S Mt. Fisht, $1650 \mathrm{~m}$, forest litter $43^{\circ} 55^{\prime} 14^{\prime \prime} \mathrm{N}, 39^{\circ} 51^{\prime} 26^{\prime \prime} \mathrm{E}$, 12.VII.2011, V. Assing" (cAss); $10^{*}$ : "Borcka, Asm., N.O. 1500 m, VI.1972, leg. F. Schubert” (NHMW).

Etymology: The specific epithet (Latin, adjective: straight) alludes to the shape of the crista apicalis of the aedeagus.

Description: Body length 2.2-2.8 mm; length of forebody $1.1-1.3 \mathrm{~mm}$. Habitus as in Fig. 59. Coloration: body reddish to reddish-brown with the head sometimes

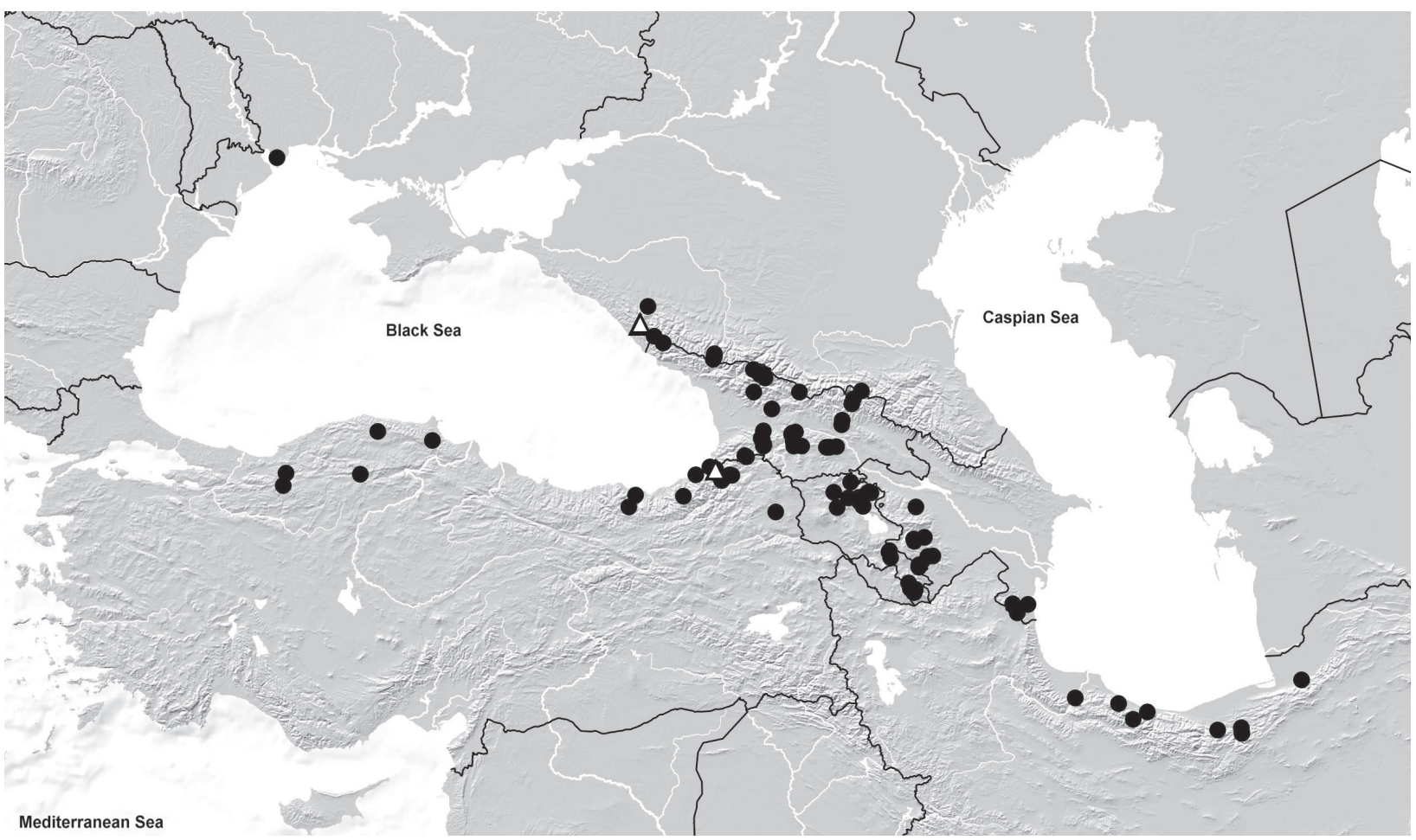

Map 7: Distributions of Oxypoda caucasica (black circles) and O. derecta (white triangles) in the Caucasus region, based on revised records. 


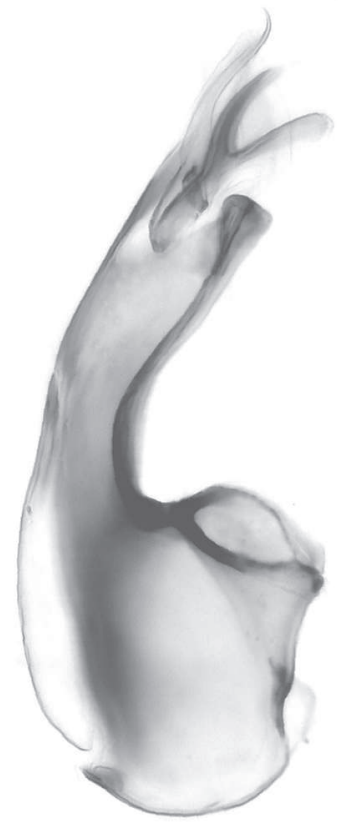

55

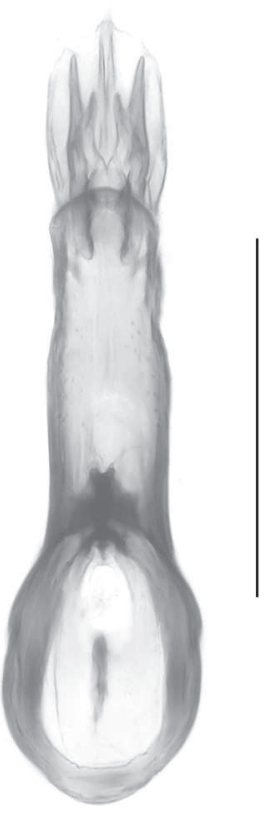

56

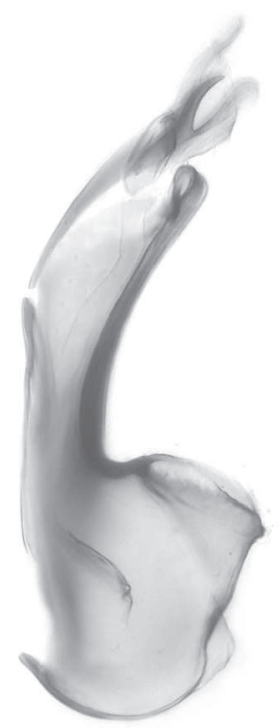

61

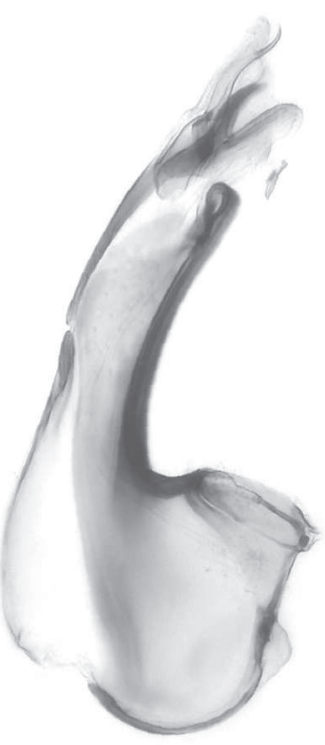

62

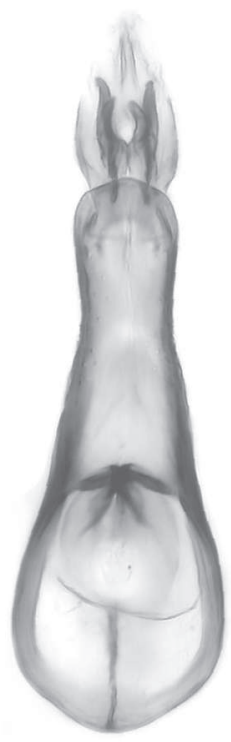

63

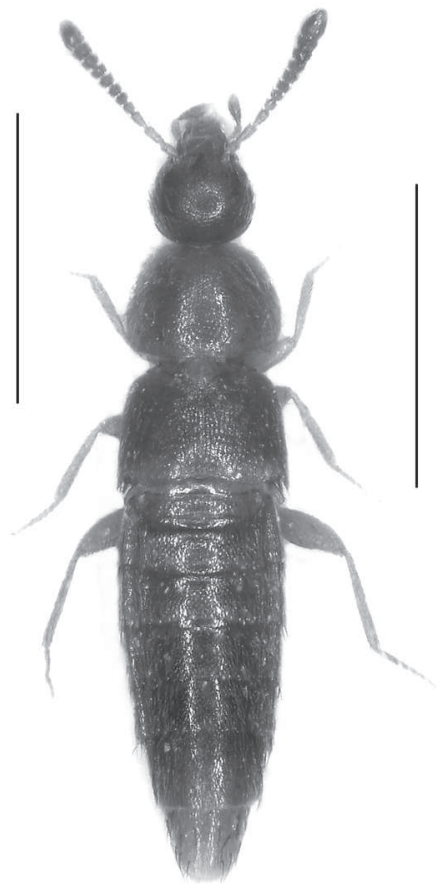

59

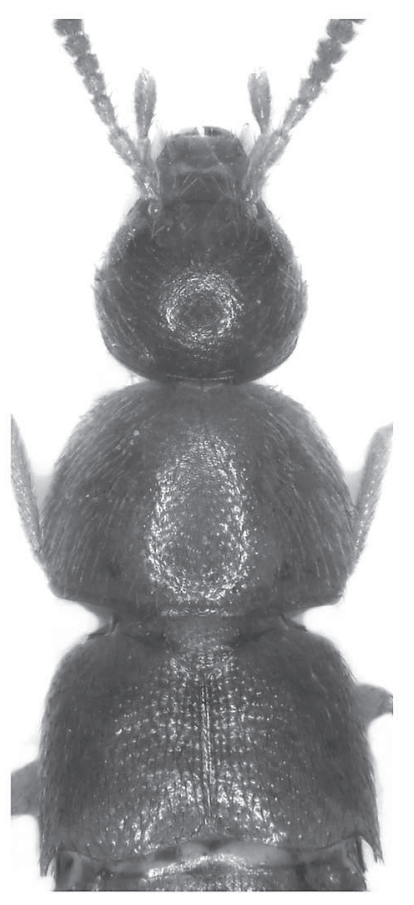

60
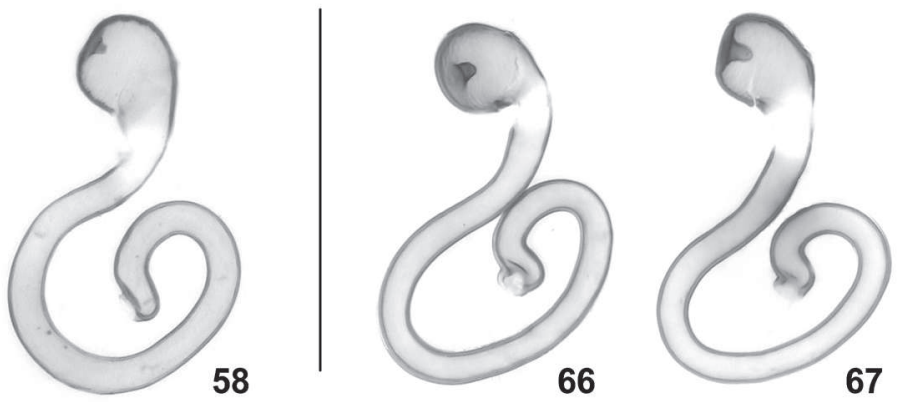

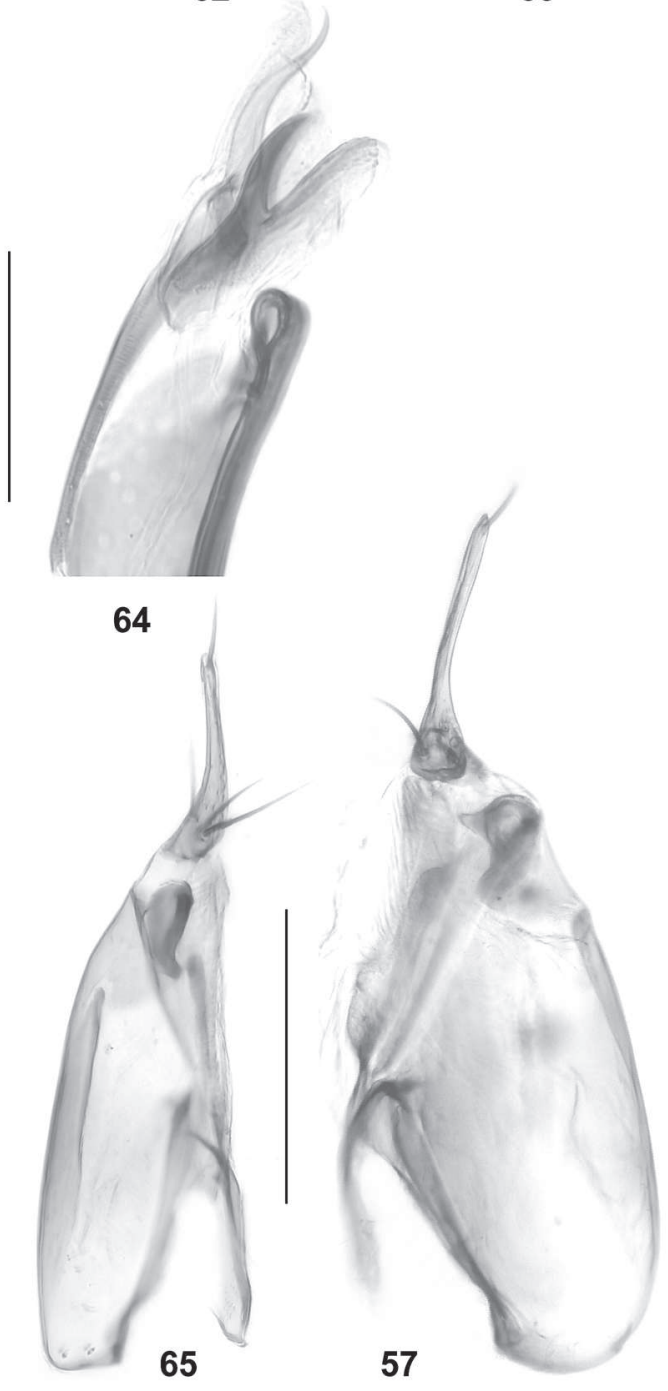

Figs 55-67: Oxypoda caucasica (55-58) and O. derecta (59-67): median lobe of aedeagus in lateral and in ventral view (55-56, 61-63); paramere $(57,65)$; spermatheca $(58,66-67)$; habitus $(59)$; forebody $(60)$; apex of median lobe of aedeagus in lateral view (64). Scale bars: 59: $1.0 \mathrm{~mm}$; 60: $0.5 \mathrm{~mm}$; 55-58, 61-63, 65-67: $0.2 \mathrm{~mm}$; 64: $0.1 \mathrm{~mm}$. 
slightly darker and with tergite VI and the anterior portion of tergite VII infuscate; legs yellowish; antennae reddish with the basal 2-3 antennomeres yellowish-red.

Head (Fig. 60) wedge-shaped; punctation fine and dense; interstices with microreticulation. Eyes flat, slightly shorter than postocular portion of head in dorsal view. Antenna approximately $0.6 \mathrm{~mm}$ long; antennomeres IV weakly transverse, $\mathrm{V}-\mathrm{X}$ of gradually increasing width and increasingly transverse, VII-X approximately twice as broad as long.

Pronotum (Fig. 60) approximately 1.35 times as broad as long and nearly 1.5 times as broad as head; punctation dense, more distinct than that of head; interstices with superficial microreticulation.

Elytra (Fig. 60) short, approximately 0.7 times as long as pronotum; punctation dense, slightly more distinct and less dense than that of pronotum; interstices with superficial microreticulation. Hind wings completely reduced. Metatarsomere I approximately as long as the combined length of metatarsomeres II-IV, or slightly longer.

Abdomen: punctation fine and dense, nearly as dense on tergite VII as on anterior tergites; interstices with shallow microreticulation; posterior margin of tergite VII with narrow palisade fringe; posterior margin of tergite VIII strongly convex.

$0^{7}$ : posterior margin of sternite VIII broadly convex; median lobe of aedeagus (Figs 61-64) approximately $0.30 \mathrm{~mm}$ long; ventral process very weakly curved and apically slightly dilated in lateral view, moderately broad and apically weakly convex in ventral view; crista apicalis long, straight, and narrow; paramere (Fig. 65) approximately $0.5 \mathrm{~mm}$ long; apical lobe weakly dilated basally, slightly less than half as long as basal portion.

\%: posterior margin of sternite VIII truncate; spermatheca (Figs 66-67) with rather long and slender proximal portion and with minute apical cuticular invagination.

Intraspecific variation: The paratype from Northeast Anatolia differs from the specimens from the West Caucasus by slightly shorter elytra (approximately 0.65 times as long as pronotum) and by a somewhat broader crista apicalis of the aedeagus. However, since no additional distinguishing characters were found, these differences are attributed to intra- rather than interspecific variation.

Comparative notes: Oxypoda derecta is distinguished from other West Palaearctic consubgeners (except for the Canarian species) by shorter elytra, completely reduced hind wings, and by the morphology of the aedeagus. As can be inferred from the similar general morphology of the aedeagus (relatively broad ventral process in ventral view; long crista apicalis; similar internal structures) and from the similar structure of the spermatheca (long and thin proximal portion; small apical cuticular invagination), it is most closely related to O. caucasica, from which it additionally differs by slightly smaller size, on average paler coloration, a smaller aedeagus with a less strongly curved and apically less distinctly dilated ventral process (lateral view) and with a much narrower crista apicalis.

Distribution and natural history: The species is currently known only from few localities in the West Caucasus and in the extreme northeast of Turkey (Maps 1,7). The altitudes range from 560 to $1650 \mathrm{~m}$. The specimens from the West Caucasus were sifted from litter and dead wood in a forest with Fagus orientalis and Castanea sativa and in a beech forest with scattered fir.

\section{Species excluded from Baeoglena}

Oxypoda recondita KRAATZ, 1856 has been assigned to Baeoglena at least since Bernhauer (1902) and is still listed in this subgenus in the Palaearctic Catalogue (Schülke \& SMetana 2015). The species shares with Baeoglena species the elongate maxillary palpi, but differs from them in other respects, particularly in the structure of the primary and secondary sexual characters. Based on the morphology of the aedeagus and the spermatheca, as well as on external characters (habitus; abdomen weakly narrowed posteriorly; punctation), O. recondita is closely allied to some species of the subgenus Bessopora Thomson, 1859, e.g., O. bicolor Mulsant \& Rey, 1853. Consequently, it can be concluded that the shape of the maxillary palpi represents a convergent condition and $O$. recondita is moved to Bessopora.

In the original description of Oxypoda dalmatina, which is based on few syntypes from "Radostak" (Montenegro), BERNHAUER (1905) states that the species is similar to O. recondita and characterized by long and robust antennae. It can thus be inferred that the species does not belong to Baeoglena, but, like O. recondita, to the subgenus Bessopora. Unfortunately, the types were not among the material from the Bernhauer collection forwarded to me by L. Zerche.

\section{Acknowledgements}

The colleagues indicated in the material section arranged loans of material from the collections under their care. Benedikt Feldmann (Münster) proof-read the manuscript.

\section{References}

Assing, V. 2003: A revision of Othiini. XIII. Horizontal and vertical distribution of Othius, new species, and additional records (Coleoptera: Staphylinidae: Staphylininae). - Entomological Problems 33 (1-2): 69-88. - https://www.zobodat.at/pdf/KOR_83_2013 _0073-0092.pdf. 
Assing, V. 2005: The first subanophthalmous species of Oxypoda Mannerheim from the Canary Islands (Coleoptera: Staphylinidae: Aleocharinae). - Vieraea 33: 151-154. - http://www.azoresbioportal.angra.uac. pt/files/publicacoes_Assing05-TheFirstSubanophtha lmousSpeciesOfOxypodaMANNERHEIMFromThe CanaryIslands.pdf.

Assing, V. 2013: On the Staphylinidae (Coleoptera) of Crete, Greece. - Stuttgarter Beiträge zur Naturkunde A, Neue Serie 6: 83-102. - https://www. naturkundemuseum-bw.de/sites/default/files/ publikationen/serie-a/ans06-05_assing-kreta.pdf.

Assing, V. 2017: On the Staphylinidae of the Greek island Ikaría, with supplementary notes on the fauna of Samos (Coleoptera: Staphylinidae). - Koleopterologische Rundschau 87: 89-116. - https://www. zobodat.at/pdf/KOR_87_2017_0089-0116.pdf.

Assing, V. \& Schülke, M. 2019: The Staphylinidae of Armenia and Nagorno-Karabakh (Coleoptera). Contributions to Entomology 69 (1): 91-173.

BARANOWski, R. \& Sörensson, M. 1978: Fångst av skalbaggar vid en översvämning vid Häckeberga i Skåne. - Entomologisk Tidskrift 99: 19-23. - http:// www.sef.nu/download/entomologisk_tidskrift/ et_1978/ET-1978-19-23o.pdf.

Bernhauer, M. 1899: Sechste Folge neuer Staphyliniden aus Europa, nebst Bemerkungen. - Verhandlungen der Kaiserlich-Königlichen Zoologisch-Botanischen Gesellschaft in Wien 49: 422-437.

Bernhauer, M. 1902: Die Staphyliniden der paläarktischen Fauna. I. Tribus: Aleocharini. (II. Theil). - Verhandlungen der Kaiserlich-Königlichen Zoologisch-Botanischen Gesellschaft in Wien 52 (Beiheft): 87-284.

Bernhauer, M. 1905: 13. Folge neuer Staphyliniden der paläarktischen Fauna, nebst Bemerkungen. - Verhandlungen der Kaiserlich-Königlichen Zoologisch-Botanischen Gesellschaft in Wien 55: 580-596.

Büche, B. 1994: Zur Käferfauna (Coleoptera) der Dünengebiete bei Sandhausen. - Beihefte zu den Veröffentlichungen für Naturschutz und Landschaftspflege in Baden-Württemberg 80: 255-282.

Erichson, W. F. 1839: Genera et species Staphylinorum insectorum coleopterorum familiae. Erster Band; pp. 1-400. - F.H. Morin, Berlin: 954 pp.

FAGEL, G. 1958: Contribution à la connaissance des Staphylinidae. LII. Sur quelques espèces du bassin méditerranéen. - Bulletin et Annales de la Société Royale d'Entomologie de Belgique 94(9-10): 232-248.

FAGEL, G. 1965: Contribution à létude des Staphylinidae. XCI. Quelques Aleocharinae nouveaux du Liban. Bulletin et Annales de la Société Royale d'Entomologie de Belgique 101 (13): 251-263.

Fauvel, A. 1895: Notes synonymiques. - Revue d'Entomologie 14 (4-5): 92-127.
Grundmann, B. 1991: Die Coleopterenfauna des Oppenweher Moores. - Berichte des Naturwissenschaftlichen Vereins für Bielefeld und Umgegend 32: 77-123.

Hansen, V. 1964: Fortegnelse over Danmarks biller (Coleoptera). - Entomologiske Meddelser 33: 1-507.

Horion, A. 1967: Faunistik der mitteleuropäischen Käfer. Bd. XI: Staphylinidae, 3. Teil: Habrocerinae bis Aleocharinae (ohne Subtribus Athetae). Überlingen-Bodensee: 419 pp.

Jörger, J. B. 1924: Beitrag zur Staphyliniden-Fauna der Schweiz. - Mitteilungen der Schweizerischen Entomologischen Gesellschaft 13: 365-368.

KocH, K. 1978: Zweiter Nachtrag zur Käferfauna der Rheinprovinz. - Decheniana (Bonn) 131: 228-261.

KöHLER, F. 1988: Die Veränderung der Käferfauna des Worringer Bruches im Kölner Norden. - Decheniana (Bonn) 141: 145-189.

LatTin, G. DE 1967: Grundriss der Zoogeographie. Gustav Fischer Verlag, Stuttgart: 602 pp.

Lohse, G. A. 1967: Die Aleocharini (s. lat.) des Niederelbegebietes und Schleswig-Holsteins (Col. Staphylinidae). - Verhandlungen des Vereins für Naturwissenschaftliche Heimatforschung, Hamburg 36: 39-50.

Lohse, G. A. 1974: Tribus 15-19 (SchistogeniniAleocharini). - In: Freude, H.; Harde, K. W. \& Lohse, G. A. (eds.): Die Käfer Mitteleuropas, Band 5, Staphylinidae II (Hypocyphtinae und Aleocharinae), Pselaphidae. - Goecke \& Evers, Krefeld: pp. 221-292.

LoHse, G. A. 1989: Ergänzungen und Berichtigungen zu Band 5. 23. Familie Staphylinidae (II) (Aleocharinae); pp. 185-240. - In: Lohse, G. A. \& Lucht, W. H. (eds.), Die Käfer Mitteleuropas. 1. Supplementband mit Katalogteil. - Goecke \& Evers, Krefeld: 346 pp.

Mulsant, E. \& Rey, C. 1875: Pp. 163-565. - In: Histoire naturelle des coléoptères de France. Brévipennes. Aleochariens (Suite). Aleocharaires. - Deyrolle, Paris: 565 pp.

Rose, A. 2000: Die Kurzflügelkäfer der Sammlung F. und R. Struve von der Nordseeinsel Borkum (Coleoptera, Staphylinidae). - Entomologische Blätter 96 (2): 127-156.

Scheerpeltz, O. 1963: Ergebnisse der von Wilhelm Kühnelt nach Griechenland unternommenen zoologischen Studienreisen. I. (Coleoptera-Staphylinidae). - Sitzungsberichte der Österreichischen Akademie der Wissenschaften, MathematischNaturwissenschaftliche Klasse, Abt. I, 172 (9-10): 413-452. - https://www.zobodat.at/pdf/SBAWW 172_0413-0452.pdf.

Schülke, M. \& Smetana, A. 2015: Staphylinidae, pp. 304-1134. - In: LöвL, I. \& LöвL, D. (eds): Catalogue of Palaearctic Coleoptera. Volume 2. Hydrophiloidea - Staphylinoidea. Revised and updated edition. - Brill, Leiden: xxvi + 1702 pp. 
Smetana, A. 2004: Staphylinidae, subfamilies Omaliinae-Dasycerinae, Phloecharinae-Apaticinae, Piestinae-Staphylininae, pp. 237-272, 329-495, 505698. - In: Löbl, I. \& Smetana, A. (eds), Catalogue of Palaearctic Coleoptera. II. Hydrophiloidea-Histeroidea-Staphylinoidea. - Apollo Books, Stenstrup: 942 pp.

SMOLEŃSKI, M. 1997: Epigeic staphylinid communities (Coleoptera: Staphylinidae) in primary succession on coastal moving dunes of the Slowiński National Park. - Polskie Pismo Entomologiczne 66: 45-81.

Thomson, C. G. 1867: Skandinaviens Coleoptera, synoptiskt bearbetade. Supplementum. Tom. IX. Lundbergska Boktryckeriet, Lund: 407 pp.

Tronquet, M. 1999: Sur quelques Oxypoda des collections A. Fauvel et G. Fagel; Derocala lucida n. sp. (Coleoptera, Staphylinidae). - Bulletin de la Société Entomologique de France 104 (2): 167-181.

Tronquet, M. 2006: Catalogue iconographique des coléoptères des Pyrénées-Orientale s. Volume I (édition revue et augmentée). Staphylinidae. - Revue de l'Association Roussillonnaise d'Entomologie 15 (Supplément): 1-127 + 78 plates.
Williams, S. A. 1990: Oxypoda praecox ERICHSON (Coleoptera: Staphylinidae) an addition to the British fauna. - British Journal of Entomology and Natural History 3: 114.

Zerche, L. 1994: Die Revision der Oxypoda-Typen aus der Sammlung Claudius Rey im Musée Guimet d'Histoire naturelle de Lyon und einiger anderer Typen der Gattung sowie die Beschreibung von vier neuen Oxypoda-Arten (Coleoptera: Staphylinidae: Aleocharinae). - Coleoptera Schwanfelder Coleopterologische Mitteilungen 6: 1-36.

Zerche, L. 1996: Die Oxypoda-Arten der Kanarischen Inseln: Taxonomie, Bionomie, Phylogenie und Biogeographie (Coleoptera: Staphylinidae: Aleocharinae). - Beiträge zur Entomologie, Berlin 46 (2): 277-372. - https://www.contributions-toentomology.org/article/view/1438/1437. 